\title{
Introduction to Early Universe Cosmology
}

\author{
Robert H. Brandenberger*t \\ Physics Department, McGill University, Montreal, Quebec, H3A 2T8, Canada \\ E-mail: rhb@physics.mcgill.ca
}

\begin{abstract}
Observational cosmology is in its "golden age" with a vast amount of recent data on the distribution of matter and light in the universe. This data can be used to probe theories of the very early universe. It is small amplitude cosmological fluctuations which encode the information about the very early universe and relate it to current data. Hence, a central topic in these lectures is the "theory of cosmological perturbations", the theory which describes the generation of inhomogeneities in the very early universe and their evolution until the current time. I will apply this theory to three classes of models of the very early universe. The first is "Inflationary Cosmology", the current paradigm for understanding the early evolution of the universe. I will review the successes of inflationary cosmology, but will also focus on some conceptual challenges which inflationary cosmology is facing, challenges which motivate the search for possible alternatives. I will introduce two alternative scenarios, the "Matter Bounce" model and "String Gas Cosmology", and I will discuss how cosmological fluctuations which can explain the current data are generated in those models.
\end{abstract}

4th International Conference on Fundamental Interactions -ICFI2010,

August 1-7, 2010

ViÃ ğosa, Brazil

\footnotetext{
${ }^{*}$ Speaker.

${ }^{\dagger}$ An earlier version of these lectures was delivered at the Observatorio Nacional in November 2009 [1].
} 


\section{Introduction}

\subsection{Overview}

Observational cosmology is currently in its "Golden Age". A wealth of new observational results are being uncovered. The cosmic microwave background (CMB) has been measured to high precision, the distribution of visible and of dark matter is being mapped out to greater and greater depths. New observational windows to probe the structure of the universe are opening up. To explain these observational results it is necessary to consider processes which happened in the very early universe.

The inflationary scenario [2] (see also [3, 4, 5]) is the current paradigm for the evolution of the very early universe. Inflation can explain some of the puzzles which the previous paradigm - Standard Big Bang cosmology - could not address. More importantly, however, inflationary cosmology gave rise to the first explanation for the origin of inhomogeneities in the universe based on causal physics [6] (see also [7, 5, 8])). The theory of structure formation in inflationary cosmology predicted the detailed shape of the angular power spectrum of CMB anisotropies, a prediction which was verified many years later observationally [9].

In spite of this phenomenological success, inflationary cosmology is not without its conceptual problems. These problems motivate the search for alternative proposals for the evolution of the early universe and for the generation of structure. These alternatives must be consistent with the current observations, and they must make predictions with which they can be observationally distinguished from inflationary cosmology. In these lectures I will discuss two alternative scenarios, the "Matter Bounce" (see e.g. [10]) and "String Gas Cosmology" [11].

I begin these lectures with a brief survey of the Standard Big Bang (SBB) model and its problems. Possibly the most important problem is the absence of a structure formation scenario based on causal physics. I will then introduce inflationary cosmology and the two alternatives and show how they address the conceptual problems of the SBB model, in particular how structure is formed in these models.

Since the information about the early universe is encoded in the spectrum of cosmological fluctuations about the expanding background, it is these fluctuations which provide a link between the physics in the very early universe and current cosmological observations. Section 2 is devoted to an overview of the theory of linearized cosmological perturbations, the main technical tool in modern cosmology. The analysis of this section is applicable to all early universe theories. In Section 3 I give an overview of inflationary cosmology, focusing on the basic principles and emphasizing recent progress and conceptual problems.

The conceptual problems of inflationary cosmology motivate the search for alternatives. In Section 4 the "Matter Bounce" alternative is discussed, in Section 5 the "String Gas" alternative.

\subsection{Standard Big Bang Model}

Standard Big Bang cosmology is based on three principles. The first is the assumption that space is homogeneous and isotropic on large scales. The second is the assumption that the dynamics of space-time is described by the Einstein field equations. The third basis of the theory is that matter can be described as a superposition of two classical perfect fluids, a radiation fluid with relativistic equation of state and pressure-less (cold) matter. 
The first principle of Standard Cosmology implies that the metric takes the following most general form for a homogeneous and isotropic four-dimensional universe

$$
d s^{2}=d t^{2}-a(t)^{2} d \mathbf{x}^{2},
$$

where $t$ is physical time, $\mathbf{x}$ denote the three co-moving spatial coordinates (points at rest in an expanding space have constant co-moving coordinates), and the scale factor $a(t)$ is proportional to the radiius of space. For simplicity we have assumed that the universe is spatially flat.

The expansion rate $H(t)$ of the universe is given by

$$
H(t)=\frac{\dot{a}}{a},
$$

where the overdot represents the derivative with respect to time. The cosmological redshift $z(t)$ at time $t$ yields the amount of expansion which space has undergone between the time $t$ and the present time $t_{0}$ :

$$
z(t)+1=\frac{a\left(t_{0}\right)}{a(t)} .
$$

In the case of a homogeneous and isotropic metric (1.1), the Einstein equations which describe how matter induces curvature of space-time reduce to a set of ordinary differential equations, the Friedmann equations. Written for simplicity in the case of no cosmological constant and no spatial curvature they are

$$
H^{2}=\frac{8 \pi G}{3} \rho,
$$

where $\rho$ is the energy density of matter and $G$ is Newton's gravitational constant, and

$$
\frac{\ddot{a}}{a}=-\frac{4 \pi G}{3}(\rho+3 p)
$$

or equivalently

$$
\dot{\rho}=-3 H(\rho+p),
$$

where $p$ is the pressure density of matter.

The third principle of Standard Cosmology says that the energy density and pressure are the sums of the contributions from cold matter (symbols with subscript "m") and radiation (subscripts "r"):

$$
\begin{aligned}
& \rho=\rho_{m}+\rho_{r} \\
& p=p_{m}+p_{r}
\end{aligned}
$$

with $p_{m}=0$ and $p_{r}=1 / 3 \rho_{r}$.

The first principle of Standard Cosmology, the homogeneity and isotropy of space on large scales, was initially introduced soleley to simplify the mathematics. However, it is now very well confirmed observationally by the near isotropy of the cosmic microwave background and by the convergence to homogeneity in the distribution of galaxies as the length scale on which the universe is probed increases.

Standard Cosmology rests on three observational pillars. Firstly, it explains Hubble's redshiftdistance relationship. Secondly, and most importantly for the theme of these lectures, it predicted 


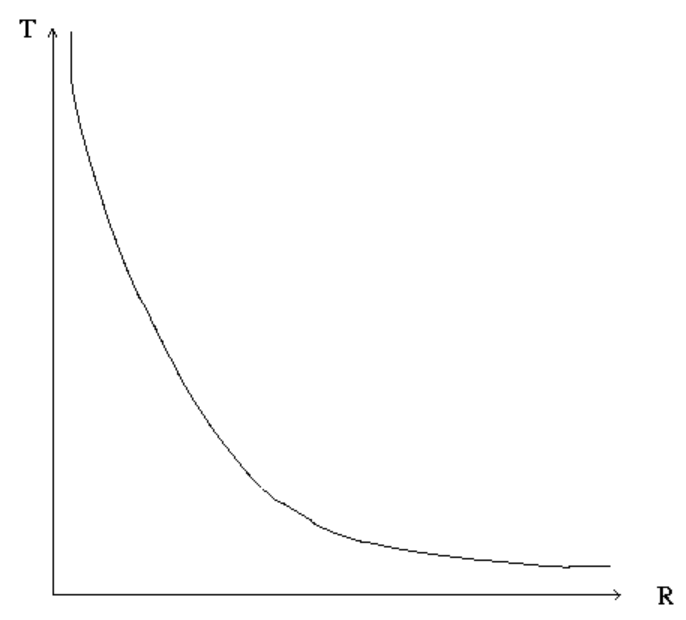

Figure 1: A sketch of the temperature (vertical axis) - time (horizontal axis) relation in Standard Cosmology. As the beginning of time is approached, the temperature diverges.

the existence and black body nature of the cosmic microwave background (CMB). The argument is as follows. If we consider regular matter and go back in time, the temperature of matter increases. Eventually, it exceeds the ionization temperature of atoms. Before that time, matter was a plasma, and space was permeated by a thermal gas of photons. A gas of photons will thus remain at the current time. The photons last scatter at a time $t_{\text {rec }}$, the "time of recombination" which occurs at a redshift of about $10^{3}$. After that, the gas of photons remains in a thermal distribution with a temperature $T$ which redshifts as the universe expands, i.e. $T \sim a^{-1}$. The CMB is this remnant gas of photons from the early universe. The precision measurement of the black body nature of the CMB [12] can be viewed as the beginning of the "Golden Age" of observational cosmology. The third observational pillar of Standard Cosmology is the good agreement between the predicted abundances of light elements and the observed ones. This agreement tells us that Standard Cosmology is a very good description of how the universe evolved back to the time of nucleosynthesis. Any modifications to the time evolution of Standard Cosmology must take place before then.

At the present time $t_{0}$ matter is dominated by the cold pressureless component. Thus, as we go back in time, the universe is (except close to the present time when it appears that a residual cosmological constant is beginning to dominate the cosmological dynamics) initially in a matterdominated phase during which $a(t) \sim t^{2 / 3}$. Since the energy density in cold matter scales as $a^{-3}$ whereas that of relativistic radiation scales as $a^{-4}$, there will be a time before which the energy density in radiation was larger than that of cold matter. The time when the two energy densities are equal is $t_{e q}$ and corresponds to a redshift of around $z=10^{4}$. For $t<t_{e q}$ the universe is radiationdominated and $a(t) \sim t^{1 / 2}$. As we go back into the past the density and temperature increase without bound and a singularity is reached at which energy density, temperature and curvature are all infinite. This is the "Big Bang" (see Fig. 1 for a sketch of the temperature/time relationship in Standard Cosmology).

\subsection{Problems of the Standard Big Bang}

Obviously, the assumptions on which Standard Cosmology is based break down long before 


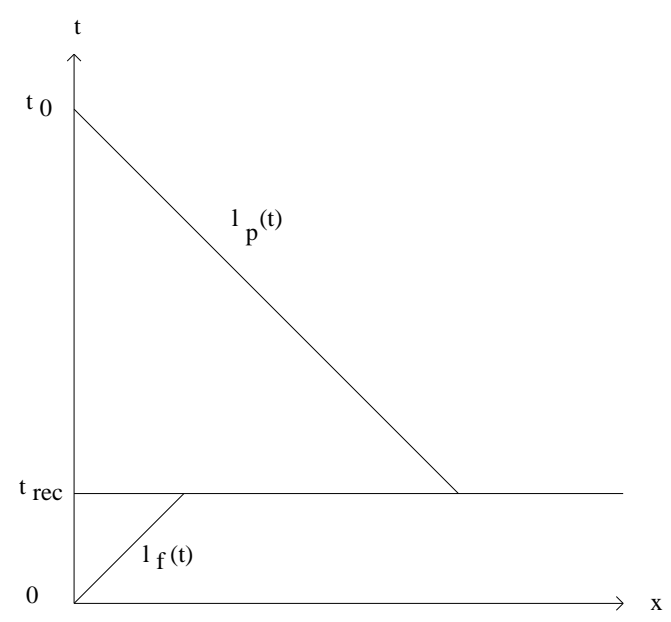

Figure 2: Sketch illustrating the horizon problem of Standard Cosmology: our past horizon at $t_{\text {rec }}$ is larger than the causal horizon (forward light cone) at that time, thus making it impossible to causally explain the observed isotropy of the CMB.

the singularity is reached. This is the "singularity problem" of the model, a problem which also arises in inflationary cosmology. It is wrong to say that Standard Cosmology predicts a Big Bang. Instead, one should say that Standard Cosmology is incomplete and one does not understand the earliest moments of the evolution of the universe.

There are, however, more "practical" "problems" of the Standard Big Bang model - problems in the sense that the model is unable to explain certain key features of the observed universe. The first such problem is the "horizon problem" [2]: within Standard Cosmology there is no possible explanation for the observed homogeneity and isotropy of the CMB. Let us consider photons reaching us from opposite angles in the sky. As sketched in Figure 2, the source points for these photons on the last scattering surface are separated by a distance greater than the horizon at that time. Hence, there is no possible causal mechanism which can relate the temperatures at the two points.

A related problem is the "size problem": if the spatial sections of the universe are finite, then the only length scale available at the Planck time $t_{p l}$ is the Planck length. However, it we extrapolate the size of our currently observed horizon back to when the temperature was equal to the Planck temperature, then the corresponding wavelength is larger than $1 \mu \mathrm{m}$, many orders of magnitude larger than the Planck length. Standard Cosmology offers no explanation for these initial conditions.

The third mystery of Standard Cosmology concerns the observed degree of spatial flatness of the universe. At the current time the observed energy density equals the "critical" energy density $\rho_{c}$ of a spatially flat universe to within $10 \%$ or better. However, in Standard Cosmology $\rho=\rho_{c}$ is an unstable critical point in an expanding universe. As the universe expands, the relative difference between $\rho$ and $\rho_{c}$ increases. This can be seen by taking the Friedmann equation in the presence of spatial curvature

$$
H^{2}+\varepsilon T^{2}=\frac{8 \pi G}{3} \rho
$$




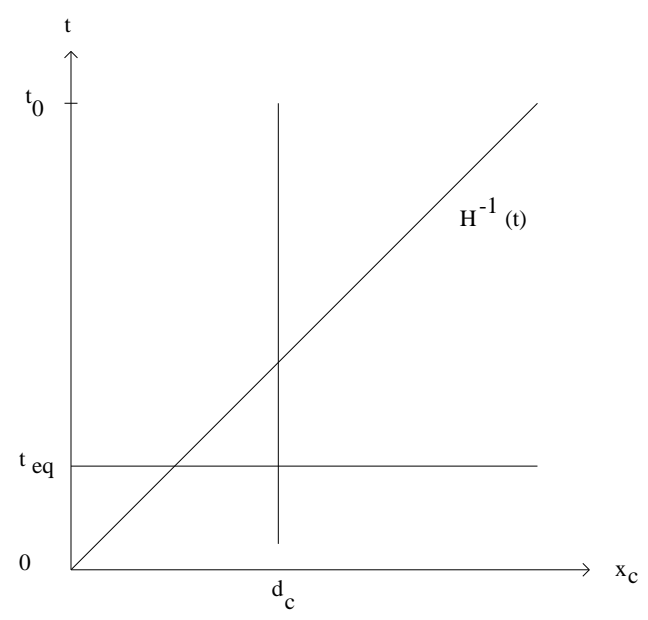

Figure 3: Sketch illustrating the formation of structure problem of Standard Cosmology: the physical wavelength of fluctuations on fixed comoving scales which correspond to the large-scale structures observed in the universe is larger than the horizon at the time $t_{e q}$ of equal matter and radiation, the time when matter fluctuations begin to grow. Hence, it is impossible to explain the origin of non-trivial correlations of the seeds for the fluctuations which had to have been present at that time.

where

$$
\varepsilon=\frac{k}{(a T)^{2}}
$$

$T$ being the temperature and $k$ the curvature constant which is $k= \pm 1$ or $k=0$ for closed, open or flat spatial sections, and comparing (1.8) with the corresponding equation in a spatially flat universe ( $k=0$ and $\rho_{c}$ replacing $\rho$. If entropy is conserved (as it is in Standard Cosmology) then $\varepsilon$ is constant and we obtain

$$
\frac{\rho-\rho_{c}}{\rho_{c}}=\frac{3}{8 \pi G} \frac{\varepsilon T^{2}}{\rho_{c}} \sim T^{-2} .
$$

Hence, to explain the currently observed degree of spatial flatness, the initial spatial curvature had to have been tuned to a very high accuracy. This is the "flatness problem".

We observe highly non-random correlations in the distribution of galaxies in the universe. The only force which can act on the relevant distances is gravity. Gravity is a weak force, and therefore the seed fluctuations which develop into the observed structures had to have been non-randomly distributed at the time $t_{e q}$, the time when gravitational clustering begins (see Section 2). However, as illustrated in Figure 3, the physical length corresponding to the fluctuations which we observe today on the largest scales (they have constant comoving scale) is larger than the horizon at that time. Hence, there can be no causal generation mechanism for these perturbations ${ }^{1}$. This is the "fluctuation problem" of Standard Cosmology.

There are also more conceptual problems: Standard Cosmology is based on treating matter as a set of perfect fluids. However, we know that at high energies and temperatures a classical description of matter breaks down. Thus, Standard Cosmology must break down at sufficiently high energies. It does not contain the adequate matter physics to describe the very early universe. Similarly, the singularity of space-time which Standard Cosmology contains corresponds

\footnotetext{
${ }^{1}$ This is the usual textbook argument. The student is invited to find (at least) two flaws in this argument.
} 


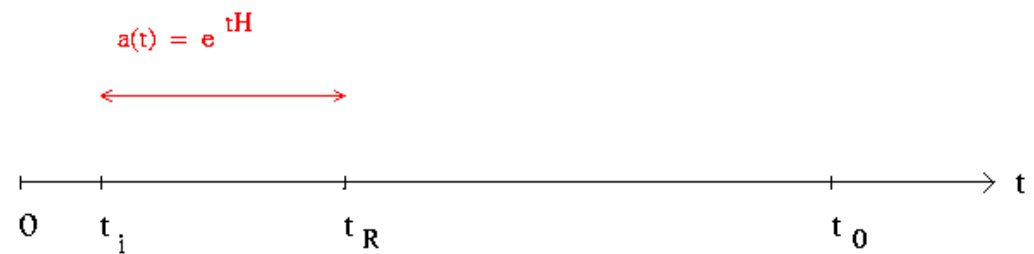

Figure 4: A sketch showing the time line of inflationary cosmology. The period of accelerated expansion begins at time $t_{i}$ and end at $t_{R}$. The time evolution after $t_{R}$ corresponds to what happens in Standard Cosmology.

to a breakdown of the assumptions on which General Relativity is based. This is the "singularity problem" of Standard Cosmology.

All of the early universe scenarios which I will discuss in the following provide solutions to the formation of structure problem. They can successfully explain the wealth of observational data on the distribution of matter in the universe and on the CMB anisotropies. The rest of these lectures will focus on this point. However, the reader should also ask under which circumstances the scenarios to be discussed below address the other problems mentioned above.

\subsection{Inflation as a Solution}

The idea of inflationary cosmology is to add a period to the evolution of the very early universe during which the scale factor undergoes accelerated expansion - most often nearly exponential growth. The time line of inflationary cosmology is sketched in Figure 4. The time $t_{i}$ is the beginning of the inflationary period, and $t_{R}$ is its end (the meaning of the subscript $R$ will become clear later). Although inflation is usually associated with physics at very high energy scales, e.g. $E \sim 10^{16} \mathrm{Gev}$, all that is required from the initial basic considerations is that inflation ends before the time of nucleosynthesis.

During the period of inflation, the density of any pre-existing particles is red-shifted. Hence, if inflation is to be viable, it must contain a mechanism to heat the universe at $t_{R}$, a "reheating" mechanism - hence the subscript $R$ on $t_{R}$. This mechanism must involve dramatic entropy generation. It is this non-adiabatic evolution which leads to a solution of the flatness problem, as the reader can verify by inspecting equation (1.10) and allowing for entropy generation at the time $t_{R}$.

A space-time sketch of inflationary cosmology is given in Figure 5 . The vertical axis is time, the horizontal axis corresponds to physical distance. Three different distance scales are shown. The solid line labelled by $k$ is the physical length corresponding to a fixed comoving perturbation. The second solid line (blue) is the Hubble radius

$$
l_{H}(t) \equiv H^{-1}(t)
$$

The Hubble radius separates scales where microphysics dominates over gravity (sub-Hubble scales) from ones on which the effects of microphysics are negligible (super-Hubble scales) ${ }^{2}$. Hence, a necessary requirement for a causal theory of structure formation is that scales we observe today originate at sub-Hubble lengths in the early universe. The third length is the "horizon", the forward

\footnotetext{
${ }^{2}$ This statement will be demonstrated later in these lectures.
} 


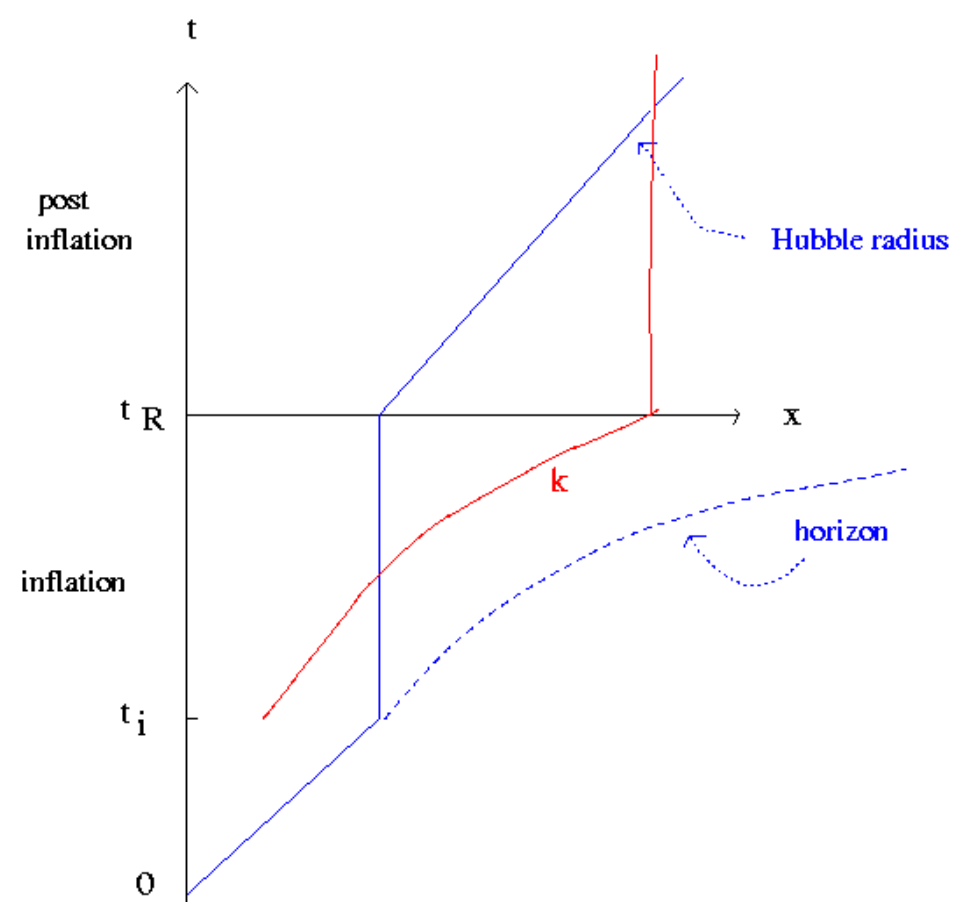

Figure 5: Space-time sketch of inflationary cosmology. The vertical axis is time, the horizontal axis corresponds to physical distance. The solid line labelled $k$ is the physical length of a fixed comoving fluctuation scale. The role of the Hubble radius and the horizon are discussed in the text.

light cone of our position at the Big Bang. The horizon is the causality limit. Note that because of the exponential expansion of space during inflation, the horizon is exponentially larger than the Hubble radius. It is important not to confuse these two scales. Hubble radius and horizon are the same in Standard Cosmology, but in all three early universe scenarios which will be discussed in the following they are different.

From Fig. 5 it is clear that provided that the period of inflation is sufficiently long, all scales which are currently observed originate as sub-Hubble scales at the beginning of the inflationary phase. Thus, in inflationary cosmology it is possible to have a causal generation mechanism of fluctuations $[6,7,5]$. Since matter pre-existing at $t_{i}$ is redshifted away, we are left with a matter vacuum. The inflationary universe scenario of structure formation is based on the hypothesis that all current structure originated as quantum vacuum fluctuations. From Figure 5 it is also clear that the horizon problem of standard cosmology can be solved provided that the period of inflation lasts sufficiently long. The reader should convince him/herself that the required period of inflation is about $50 \mathrm{H}^{-1}$ if inflation takes place at an enegy scale of about $10^{16} \mathrm{GeV}$. Inflation thus solve both the horizon and the structure formation problems.

To obtain exponential expansion of space in the context of Einstein gravity the energy density must be constant. Thus, during inflation the total energy and size of the universe both increase exponentially. In this way, inflation can solve the size and entropy problems of Standard Cosmology.

To summarize the main point concerning the generation of cosmological fluctuations (the main theme of these lectures) in inflationary cosmology: the first crucial criterium which must be satisfied in order to have a successful theory of structure formation is that fluctuation scales originate 
inside the Hubble radius. In inflationary cosmology it is the accelerated expansion of space during the inflationary phase which provides this success. In the following we will emphasize what is responsible for the corresponding success in the two alternative scenarios which we will discuss.

\subsection{Matter Bounce as a Solution}

The first alternative to cosmological inflation as a theory of structure formation is the "matter bounce" , an alternative which is not yet well appreciated (for an overview the reader is referred to [10]). The scenario is based on a cosmological background in which the scale factor $a(t)$ bounces in a non-singular manner.

Figure 6 shows a space-time sketch of such a bouncing cosmology. Without loss of generality we can adjust the time axis such that the bounce point (minimal value of the scale factor) occurs at $t=0$. There are three phases in such a non-singular bounce: the initial contracting phase during which the Hubble radius is decreasing linearly in $|t|$, a bounce phase during which a transition from contraction to expansion takes place, and thirdly the usual expanding phase of Standard Cosmology. There is no prolonged inflationary phase after the bounce, nor is there a time-symmetric deflationary contracting period before the bounce point. As is obvious from the Figure, scales which we observe today started out early in the contracting phase at sub-Hubble lengths. The matter bounce scenario assumes that the contracting phase is matter-dominated at the times when scales we observe today exit the Hubble radius. A model in which the contracting phase is the time reverse of our current expanding phase would obey this condition. The assumption of an initial matter-dominated phase will be seen later in these lectures to be important if we want to obtain a scale-invariant spectrum of cosmological perturbations from initial vacuum fluctuations $[13,14,15]$.

Let us make a first comparison with inflation. A non-deflationary contracting phase replaces the accelerated expanding phase as a mechanism to bring fixed comoving scales within the Hubble radius as we go back in time, allowing us to consider the possibility of a causal generation mechanism of fluctuations. Starting with vacuum fluctuations, a matter-dominated contracting phase is required in order to obtain a scale-invariant spectrum. This corresponds to the requirement in inflationary cosmology that the accelerated expansion be nearly exponential.

With Einstein gravity and matter satisfying the usual energy conditions it is not possible to obtain a non-singular bounce. However, as mentioned before, it is unreasonable to expect that Einstein gravity will provide a good description of the physics near the bounce. There are a large number of ways to obtain a non-singular bouncing cosmology. To mention but a few, it has been shown that a bouncing cosmology results naturally from the special ghost-free higher derivative gravity Lagrangian introduced in [16]. Bounces also arise in the higher-derivative non-singular universe construction of [17], in "mirage cosmology" (see e.g. [18]) which is the cosmology on a brane moving through a curved higher-dimensional bulk space-time (the time dependence for a brane observer is induced by the motion through the bulk), or - within the context of Einstein gravity - by making use of "quintom matter" (matter consisting of two components, one with regular kinetic term in the action, the other one with opposite sign kinetic action) [19]. For an in-depth review of ways of obtaining bouncing cosmologies see [20]. Very recently, it has been shown [21] that a bouncing cosmology can easily emerge from Horava-Lifshitz gravity [22], a new approach to quantizing gravity. 


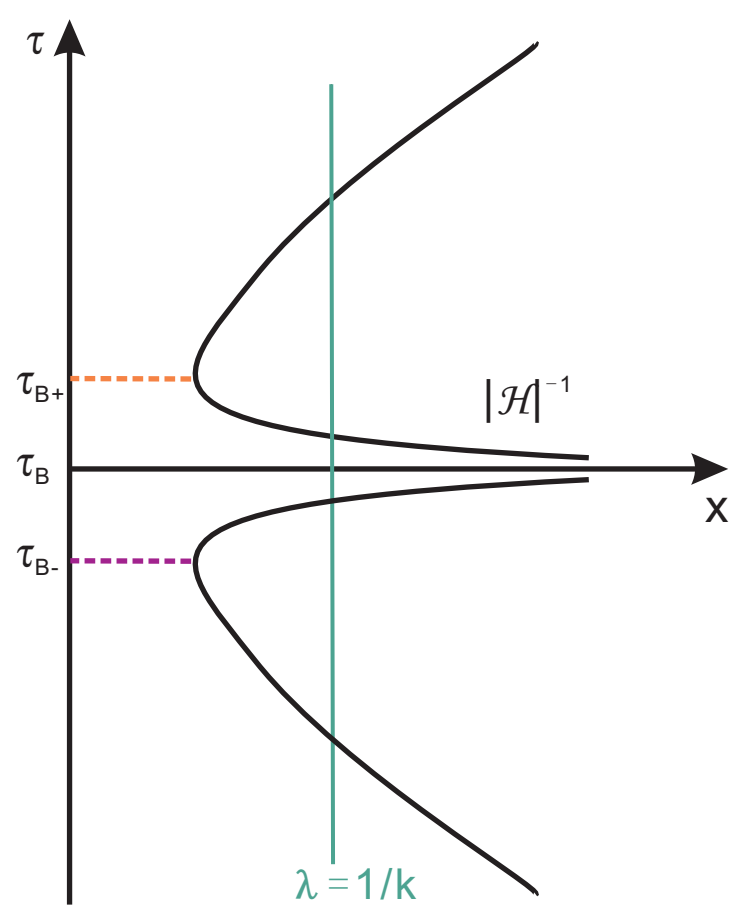

Figure 6: Space-time sketch in the matter bounce scenario. The vertical axis is conformal time $\eta$, the horizontal axis denotes a co-moving space coordinate. Also, $\mathscr{H}^{-1}$ denotes the co-moving Hubble radius.

In the matter bounce scenario the universe begins cold and therefore large. Thus, the size problem of Standard Cosmology does not arise. As is obvious from Figure 6, there is no horizon problem for the matter bounce scenario as long as the contracting period is long (to be specific, of similar duration as the post-bounce expanding phase until the present time). By the same argument, it is possible to have a causal mechanism for generating the primordial cosmological perturbations which evolve into the structures we observe today. Specifically, as will be discussed in the section of the matter bounce scenario, if the fluctuations originate as vacuum perturbations on sub-Hubble scales in the contracting phase, then the resulting spectrum at late times for scales exiting the Hubble radius in the matter-dominated phase of contraction is scale-invariant $[13,14,15]$. The propagation of infrared (IR) fluctuations through the non-singular bounce was analyzed in the case of the higher derivative gravity model of [16] in [23], in mirage cosmology in [18], in the case of the quintom bounce in $[24,25]$ and for a Horava-Lifshitz bounce in [26]. The result of these studies is that the scale-invariance of the spectrum before the bounce goes persists after the bounce as long as the time period of the bounce phase is short compared to the wavelengths of the modes being considered. Note that if the fluctuations have a thermal origin, then the condition on the background cosmology to yield scale-invariance of the spectrum of fluctuations is different [27].

\subsection{String Gas Cosmology as a Solution}

String gas cosmology [28] (see also [29], and see [11] for a comprehensive review) is a toy model of cosmology which results from coupling a gas of fundamental strings to a background space-time metric. It is assumed that the spatial sections are compact. It is argued that the universe starts in a quasi-static phase during which the temperature of the string gas hovers at the Hagedorn 


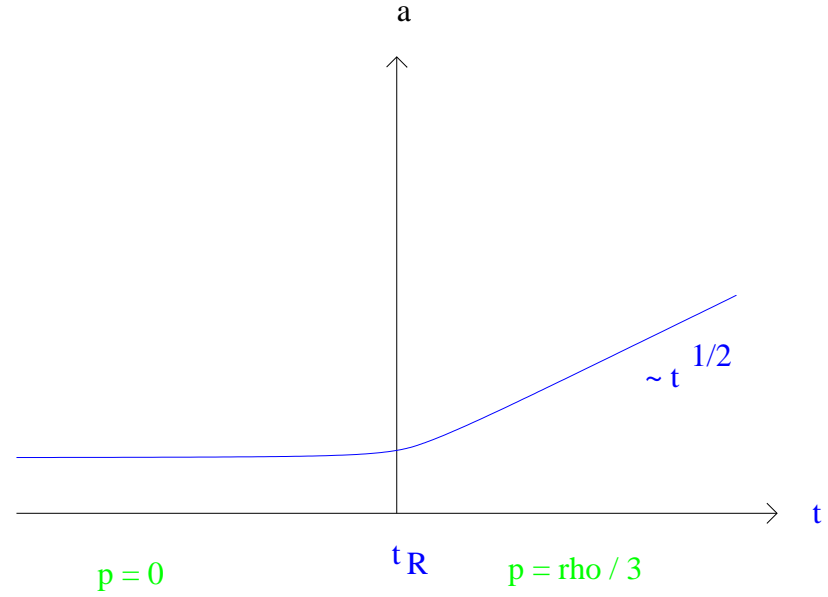

Figure 7: The dynamics of string gas cosmology. The vertical axis represents the scale factor of the universe, the horizontal axis is time. Along the horizontal axis, the approximate equation of state is also indicated. During the Hagedorn phase the pressure is negligible due to the cancellation between the positive pressure of the momentum modes and the negative pressure of the winding modes, after time $t_{R}$ the equation of state is that of a radiation-dominated universe.

value [30], the maximal temperature of a gas of closed strings in thermal equilibrium. The string gas in this early phase is dominated by strings winding the compact spatial sections. The annihilation of winding strings will produce string loops and lead to a transition from the early quasi-static phase to the radiation phase of Standard Cosmology. Fig. 7 shows a sketch of the evolution of the scale factor in string gas cosmology.

In Figure 8 we sketch the space-time diagram in string gas cosmology. Since the early Hagedorn phase is quasi-static, the Hubble radius is infinite. For the same reason, the physical wavelength of fluctuations remains constant in this phase. At the end of the Hagedorn phase, the Hubble radius decreases to a microscopic value and makes a transition to its evolution in Standard Cosmology.

Once again, we see that fluctuations originate on sub-Hubble scales. In string gas cosmology, it is the existence of a quasi-static phase which leads to this result. As will be discussed in the section on string gas cosmology, the source of perturbations in string gas cosmology is thermal: string thermodynamical fluctuations in a compact space with stable winding modes in fact leads to a scale-invariant spectrum [31].

\section{Theory of Cosmological Perturbations}

The key tool which is used in modern cosmology to connect theories of the very early universe with cosmological observations today is the theory of cosmological perturbations. In the following, we will give an overview of this theory (similar to the overview in [33] which in turn is based on the comprehensive review [34]). We begin with the analysis of perturbations in Newtonian cosmology, a useful exercise to develop intuition and notations. 


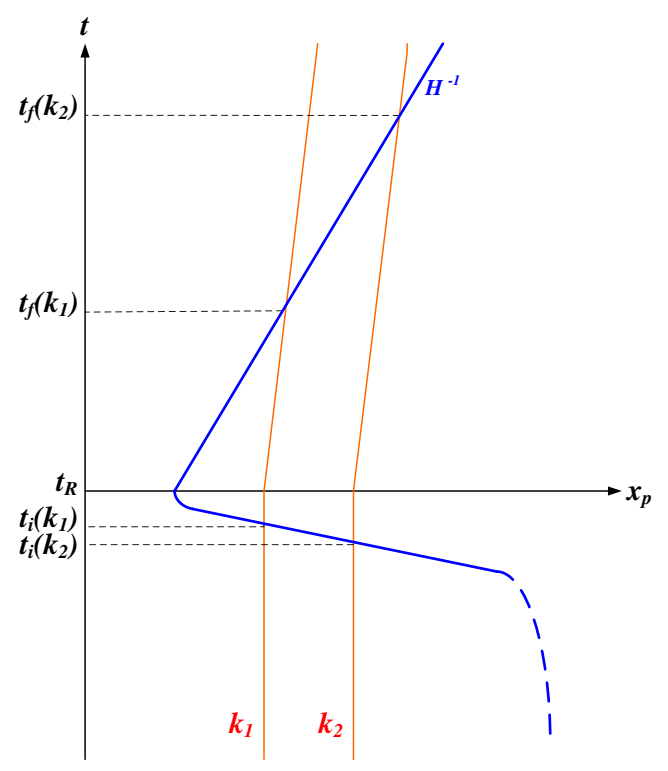

Figure 8: Space-time diagram (sketch) showing the evolution of fixed co-moving scales in string gas cosmology. The vertical axis is time, the horizontal axis is physical distance. The solid curve represents the Einstein frame Hubble radius $H^{-1}$ which shrinks abruptly to a micro-physical scale at $t_{R}$ and then increases linearly in time for $t>t_{R}$. Fixed co-moving scales (the dotted lines labeled by $k_{1}$ and $k_{2}$ ) which are currently probed in cosmological observations have wavelengths which are smaller than the Hubble radius before $t_{R}$. They exit the Hubble radius at times $t_{i}(k)$ just prior to $t_{R}$, and propagate with a wavelength larger than the Hubble radius until they reenter the Hubble radius at times $t_{f}(k)$.

\subsection{Newtonian Theory}

The growth of density fluctuations is a consequence of the purely attractive nature of the gravitational force. Imagine (first in a non-expanding background) a density excess $\delta \rho$ localized about some point $\mathbf{x}$ in space. This fluctuation produces an attractive force which pulls the surrounding matter towards $\mathbf{x}$. The magnitude of this force is proportional to $\delta \rho$. Hence, by Newton's second law

$$
\ddot{\delta} \rho \sim G \delta \rho,
$$

where $G$ is Newton's gravitational constant. Hence, there is an exponential instability of flat spacetime to the development of fluctuations.

Obviously, in General Relativity it is inconsistent to consider density fluctuations in a nonexpanding background. If we consider density fluctuations in an expanding background, then the expansion of space leads to a friction term in (2.1). Hence, instead of an exponential instability to the development of fluctuations, fluctuations in an expanding Universe will grow as a power of time. We will now determine what this power is and how it depends both on the background cosmological expansion rate and on the length scale of the fluctuations. 
We first consider the evolution of hydrodynamical matter fluctuations in a fixed non-expanding background. In this context, matter is described by a perfect fluid, and gravity by the Newtonian gravitational potential $\varphi$. The fluid variables are the energy density $\rho$, the pressure $p$, the fluid velocity $\mathbf{v}$, and the entropy density $s$. The basic hydrodynamical equations are

$$
\begin{aligned}
\dot{\rho}+\nabla \cdot(\rho \mathbf{v}) & =0 \\
\dot{\mathbf{v}}+(\mathbf{v} \cdot \nabla) \mathbf{v}+\frac{1}{\rho} \nabla p+\nabla \varphi & =0 \\
\nabla^{2} \varphi & =4 \pi G \rho \\
\dot{s}+(\mathbf{v} \cdot \nabla) s & =0 \\
p & =p(\rho, s) .
\end{aligned}
$$

The first equation is the continuity equation, the second is the Euler (force) equation, the third is the Poisson equation of Newtonian gravity, the fourth expresses entropy conservation, and the last describes the equation of state of matter. The derivative with respect to time is denoted by an over-dot.

The background is given by the energy density $\rho_{o}$, the pressure $p_{0}$, vanishing velocity, constant gravitational potential $\varphi_{0}$ and constant entropy density $s_{0}$. As mentioned above, it does not satisfy the background Poisson equation.

The equations for cosmological perturbations are obtained by perturbing the fluid variables about the background,

$$
\begin{aligned}
\rho & =\rho_{0}+\delta \rho \\
\mathbf{v} & =\delta \mathbf{v} \\
p & =p_{0}+\delta p \\
\varphi & =\varphi_{0}+\delta \varphi \\
s & =s_{0}+\delta s
\end{aligned}
$$

where the fluctuating fields $\delta \rho, \delta \mathbf{v}, \delta p, \delta \varphi$ and $\delta s$ are functions of space and time, by inserting these expressions into the basic hydrodynamical equations (2.2), by linearizing, and by combining the resulting equations which are of first order in time. We get the following differential equations for the energy density fluctuation $\delta \rho$ and the entropy perturbation $\delta s$

$$
\begin{aligned}
\ddot{\delta \rho}-c_{s}^{2} \nabla^{2} \delta \rho-4 \pi G \rho_{0} \delta \rho & =\sigma \nabla^{2} \delta s \\
\dot{\delta} s & =0,
\end{aligned}
$$

where the variables $c_{s}^{2}$ and $\sigma$ describe the equation of state

$$
\delta p=c_{s}^{2} \delta \rho+\sigma \delta S
$$

with

$$
c_{s}^{2}=\left(\frac{\delta p}{\delta \rho}\right)_{\mid s}
$$

denoting the square of the speed of sound. 
The fluctuations can be classified as follows: If $\delta s$ vanishes, we have adiabatic fluctuations. If $\delta s$ is non-vanishing but $\dot{\delta}=0$, we speak of an entropy fluctuation.

The first conclusions we can draw from the basic perturbation equations (2.4) are that

1) entropy fluctuations do not grow,

2) adiabatic fluctuations are time-dependent, and

3) entropy fluctuations seed adiabatic ones.

All of these conclusions will remain valid in the relativistic theory.

Since the equations are linear, we can work in Fourier space. Each Fourier component $\delta \rho_{k}(t)$ of the fluctuation field $\delta \rho(\mathbf{x}, t)$ evolves independently. In the case of adiabatic fluctuations, the cosmological perturbations are described by a single field which obeys a second order differential equation and hence has two fundamental solutions. We will see that this conclusion remains true in the relativistic theory.

Taking a closer look at the equation of motion for $\delta \rho$, we see that the third term on the left hand side represents the force due to gravity, a purely attractive force yielding an instability of flat space-time to the development of density fluctuations (as discussed earlier, see (2.1)). The second term on the left hand side of (2.4) represents a force due to the fluid pressure which tends to set up pressure waves. In the absence of entropy fluctuations, the evolution of $\delta \rho$ is governed by the combined action of both pressure and gravitational forces.

Restricting our attention to adiabatic fluctuations, we see from (2.4) that there is a critical wavelength, the Jeans length, whose wavenumber $k_{J}$ is given by

$$
k_{J}=\left(\frac{4 \pi G \rho_{0}}{c_{s}^{2}}\right)^{1 / 2} .
$$

Fluctuations with wavelength longer than the Jeans length $\left(k \ll k_{J}\right)$ grow exponentially

$$
\delta \rho_{k}(t) \sim e^{\omega_{k} t} \text { with } \omega_{k} \sim 4\left(\pi G \rho_{0}\right)^{1 / 2}
$$

whereas short wavelength modes $\left(k \gg k_{J}\right)$ oscillate with frequency $\omega_{k} \sim c_{s} k$. Note that the value of the Jeans length depends on the equation of state of the background. For a background dominated by relativistic radiation, the Jeans length is large (of the order of the Hubble radius $H^{-1}(t)$ ), whereas for pressure-less matter it vanishes.

Let us now improve on the previous analysis and study Newtonian cosmological fluctuations about an expanding background. In this case, the background equations are consistent (the nonvanishing average energy density leads to cosmological expansion). However, we are still neglecting general relativistic effects (the fluctuations of the metric). Such effects turn out to be dominant on length scales larger than the Hubble radius $H^{-1}(t)$, and thus the analysis of this section is applicable only to smaller scales.

The background cosmological model is given by the energy density $\rho_{0}(t)$, the pressure $p_{0}(t)$, and the recessional velocity $\mathbf{v}_{0}=H(t) \mathbf{x}$, where $\mathbf{x}$ is the Euclidean spatial coordinate vector ("physical coordinates"). The space- and time-dependent fluctuating fields are defined as in the previous section:

$$
\begin{aligned}
\rho(t, \mathbf{x}) & =\rho_{0}(t)\left(1+\delta_{\varepsilon}(t, \mathbf{x})\right) \\
\mathbf{v}(t, \mathbf{x}) & =\mathbf{v}_{0}(t, \mathbf{x})+\delta \mathbf{v}(t, \mathbf{x}) \\
p(t, \mathbf{x}) & =p_{0}(t)+\delta p(t, \mathbf{x}),
\end{aligned}
$$


where $\delta_{\varepsilon}$ is the fractional energy density perturbation (we are interested in the fractional rather than in the absolute energy density fluctuation!), and the pressure perturbation $\delta p$ is defined as in (2.5). In addition, there is the possibility of a non-vanishing entropy perturbation defined as in (2.3).

We now insert this ansatz into the basic hydrodynamical equations (2.2), linearize in the perturbation variables, and combine the first order differential equations for $\delta_{\varepsilon}$ and $\delta p$ into a single second order differential equation for $\delta \rho_{\varepsilon}$. The result simplifies if we work in "comoving coordinates" $\mathbf{q}$ which are the coordinates painted onto the expanding background, i.e. $\mathbf{x}(t)=a(t) \mathbf{q}(t)$. After some algebra, we obtain the following equation which describes the time evolution of density fluctuations:

$$
\ddot{\delta}_{\varepsilon}+2 H \dot{\delta}_{\varepsilon}-\frac{c_{s}^{2}}{a^{2}} \nabla_{q}^{2} \delta_{\varepsilon}-4 \pi G \rho_{0} \delta_{\varepsilon}=\frac{\sigma}{\rho_{0} a^{2}} \delta S
$$

where the subscript $q$ on the $\nabla$ operator indicates that derivatives with respect to comoving coordinates are used. In addition, we have the equation of entropy conservation $\dot{\delta} S=0$.

Comparing with the equations (2.4) obtained in the absence of an expanding background, we see that the only difference is the presence of a Hubble damping term in the equation for $\delta_{\varepsilon}$. This term will moderate the exponential instability of the background to long wavelength density fluctuations. In addition, it will lead to a damping of the oscillating solutions on short wavelengths. More specifically, for physical wavenumbers $k_{p} \ll k_{J}$ (where $k_{J}$ is again given by (2.7)), and in a matter-dominated background cosmology, the general solution of (2.10) in the absence of any entropy fluctuations is given by

$$
\delta_{k}(t)=c_{1} t^{2 / 3}+c_{2} t^{-1},
$$

where $c_{1}$ and $c_{2}$ are two constants determined by the initial conditions, and we have dropped the subscript $\varepsilon$ in expressions involving $\delta_{\varepsilon}$. There are two fundamental solutions, the first is a growing mode with $\delta_{k}(t) \sim a(t)$, the second a decaying mode with $\delta_{k}(t) \sim t^{-1}$. On short wavelengths, one obtains damped oscillatory motion:

$$
\delta_{k}(t) \sim a^{-1 / 2}(t) \exp \left( \pm i c_{s} k \int d t^{\prime} a^{-1}\left(t^{\prime}\right)\right) .
$$

The above analysis applies for $t>t_{e q}$, when we are following the fluctuations of the dominant component of matter. In the radiation era, cold matter fluctuations only grow logarithmically in time since the dominant component of matter (the relativistic radiation) does not cluster and hence the term in the equation of motion for fluctuations which represents the gravitational attraction force is suppressed. In this sense, we can say that inhomogeneities begin to cluster at the time $t_{e q}$.

As a simple application of the Newtonian equations for cosmological perturbations derived above, let us compare the predicted cosmic microwave background (CMB) anisotropies in a spatially flat universe with only baryonic matter - Model A - to the corresponding anisotropies in a flat Universe with mostly cold dark matter (pressure-less non-baryonic dark matter) - Model B. We start with the observationally known amplitude of the relative density fluctuations today (time $\left.t_{0}\right)$, and we use the fact that the amplitude of the CMB anisotropies on the angular scale $\theta(k)$ corresponding to the comoving wavenumber $k$ is set by the primordial value of the gravitational potential $\phi$ - introduced in the following section - which in turn is related to the primordial value of the density fluctuations at Hubble radius crossing (and not to its value at the time $t_{\text {rec }}$ - see e.g. Chapter 17 of [34]). 
In Model A, the dominant component of the pressure-less matter is coupled to radiation between $t_{e q}$ and $t_{r e c}$, the time of last scattering. Thus, the Jeans length is comparable to the Hubble radius. Therefore, for comoving galactic scales, $k \gg k_{J}$ in this time interval, and thus the fractional density contrast decreases as $a(t)^{-1 / 2}$. In contrast, in Model $\mathrm{B}$, the dominant component of pressure-less matter couples only weakly to radiation, and hence the Jeans length is negligibly small. Thus, in Model B, the relative density contrast grows as $a(t)$ between $t_{e q}$ and $t_{\text {rec }}$. In the time interval $t_{\text {rec }}<t<t_{0}$, the fluctuations scale identically in Models A and B. Summarizing, we conclude, working backwards in time from a fixed amplitude of $\delta_{k}$ today, that the amplitudes of $\delta_{k}\left(t_{e q}\right)$ in Models A and B (and thus their primordial values) are related by

$$
\left.\left.\delta_{k}\left(t_{e q}\right)\right|_{A} \simeq\left(\frac{a\left(t_{r e c}\right)}{a\left(t_{e q}\right)}\right)^{3 / 2} \delta_{k}\left(t_{e q}\right)\right|_{B} .
$$

Hence, in Model A (without non-baryonic dark matter) the CMB anisotropies are predicted to be a factor of about 30 larger [35] than in Model B, way in excess of the recent observational results. This is one of the strongest arguments for the existence of non-baryonic dark matter ${ }^{3}$. Note that the precise value of the enhancement factor depends on the value of the cosmological constant $\Lambda$ the above result holds for $\Lambda=0$.

\subsection{Observables}

Let us consider perturbations on a fixed comoving length scale given by a comoving wavenumber $k$. The corresponding physical length increases as $a(t)$. This is to be compared with the Hubble radius $H^{-1}(t)$ which scales as $t$ provided $a(t)$ grows as a power of $t$. In the late time Universe, $a(t) \sim t^{1 / 2}$ in the radiation-dominated phase (i.e. for $t<t_{e q}$ ), and $a(t) \sim t^{2 / 3}$ in the matter-dominated period $\left(t_{e q}<t<t_{0}\right)$. Thus, we see that at sufficiently early times, all comoving scales had a physical length larger than the Hubble radius. If we consider large cosmological scales (e.g. those corresponding to the observed CMB anisotropies or to galaxy clusters), the time $t_{H}(k)$ of "Hubble radius crossing" (when the physical length was equal to the Hubble radius) was in fact later than $t_{e q}$.

Cosmological fluctuations can be described either in position space or in momentum space. In position space, we compute the root mean square mass fluctuation $\delta M / M(k, t)$ in a sphere of radius $l=2 \pi / k$ at time $t$. A scale-invariant spectrum of fluctuations is defined by the relation

$$
\frac{\delta M}{M}\left(k, t_{H}(k)\right)=\text { const. . }
$$

Such a spectrum was first suggested by Harrison [36] and Zeldovich [37] as a reasonable choice for the spectrum of cosmological fluctuations. We can introduce the "spectral index" $n$ of cosmological fluctuations by the relation

$$
\left(\frac{\delta M}{M}\right)^{2}\left(k, t_{H}(k)\right) \sim k^{n-1},
$$

and thus a scale-invariant spectrum corresponds to $n=1$.

\footnotetext{
${ }^{3}$ In my opinion no proposed alternative to particle dark matter should be taken serious unless their proponents can demonstrate that their model can reproduce the agreement between the amplitudes of the CMB anisotropies on one hand and of the large-scale matter power spectrum on the other hand.
} 
To make the transition to the (more frequently used) momentum space representation, we Fourier decompose the fractional spatial density contrast

$$
\delta_{\varepsilon}(\mathbf{x}, t)=V^{1 / 2} \int d^{3} k \tilde{\delta}_{\varepsilon}(\mathbf{k}, t) e^{i \mathbf{k} \cdot \mathbf{x}},
$$

where $V$ is a cutoff volume. The power spectrum $P_{\delta}$ of density fluctuations is defined by

$$
P_{\delta}(k)=k^{3}\left|\tilde{\delta}_{\varepsilon}(k)\right|^{2},
$$

where $k$ is the magnitude of $\mathbf{k}$, and we have assumed for simplicity a Gaussian distribution of fluctuations in which the amplitude of the fluctuations only depends on $k$.

We can also define the power spectrum of the gravitational potential $\varphi$ :

$$
P_{\varphi}(k)=k^{3}|\tilde{\delta \varphi}(k)|^{2} .
$$

These two power spectra are related by the Poisson equation (2.2)

$$
P_{\varphi}(k) \sim k^{-4} P_{\delta}(k) .
$$

In general, the condition of scale-invariance is expressed in momentum space in terms of the power spectrum evaluated at a fixed time. To obtain this condition, we first use the time dependence of the fractional density fluctuation from (2.11) to determine the mass fluctuations at a fixed time $t>t_{H}(k)>t_{e q}$ (the last inequality is a condition on the scales considered)

$$
\left(\frac{\delta M}{M}\right)^{2}(k, t)=\left(\frac{t}{t_{H}(k)}\right)^{4 / 3}\left(\frac{\delta M}{M}\right)^{2}\left(k, t_{H}(k)\right) .
$$

The time of Hubble radius crossing is given by

$$
a\left(t_{H}(k)\right) k^{-1}=2 t_{H}(k),
$$

and thus

$$
t_{H}(k)^{1 / 3} \sim k^{-1} .
$$

Inserting this result into (2.20) and making use of (2.15) we find

$$
\left(\frac{\delta M}{M}\right)^{2}(k, t) \sim k^{n+3} .
$$

Since, for reasonable values of the index of the power spectrum, $\delta M / M(k, t)$ is dominated by the Fourier modes with wavenumber $k$, we find that (2.23) implies

$$
\left|\tilde{\delta}_{\varepsilon}\right|^{2} \sim k^{n},
$$

or, equivalently,

$$
P_{\varphi}(k) \sim k^{n-1} .
$$




\subsection{Classical Relativistic Theory}

\subsubsection{Introduction}

The Newtonian theory of cosmological fluctuations discussed in the previous section breaks down on scales larger than the Hubble radius because it neglects perturbations of the metric, and because on large scales the metric fluctuations dominate the dynamics.

Let us begin with a heuristic argument to show why metric fluctuations are important on scales larger than the Hubble radius. For such inhomogeneities, one should be able to approximately describe the evolution of the space-time by applying the first FRW equation (3.7) of homogeneous and isotropic cosmology to the local Universe (this approximation is made more rigorous in [38]). Based on this equation, a large-scale fluctuation of the energy density will lead to a fluctuation (" $\delta a$ ") of the scale factor $a$ which grows in time. This is due to the fact that self gravity amplifies fluctuations even on length scales $\lambda$ greater than the Hubble radius.

This argument is made rigorous in the following analysis of cosmological fluctuations in the context of general relativity, where both metric and matter inhomogeneities are taken into account. We will consider fluctuations about a homogeneous and isotropic background cosmology, given by the metric (1.1), which can be written in conformal time $\eta$ (defined by $d t=a(t) d \eta$ ) as

$$
d s^{2}=a(\eta)^{2}\left(d \eta^{2}-d \mathbf{x}^{2}\right) .
$$

The theory of cosmological perturbations is based on expanding the Einstein equations to linear order about the background metric. The theory was initially developed in pioneering works by Lifshitz [39]. Significant progress in the understanding of the physics of cosmological fluctuations was achieved by Bardeen [40] who realized the importance of subtracting gauge artifacts (see below) from the analysis (see also [41, 42]). The following discussion is based on Part I of the comprehensive review article [34]. Other reviews - in some cases emphasizing different approaches - are $[43,44,45,46]$.

\subsubsection{Classifying Fluctuations}

The first step in the analysis of metric fluctuations is to classify them according to their transformation properties under spatial rotations. There are scalar, vector and second rank tensor fluctuations. In linear theory, there is no coupling between the different fluctuation modes, and hence they evolve independently (for some subtleties in this classification, see [47]).

We begin by expanding the metric about the FRW background metric $g_{\mu \nu}^{(0)}$ given by (2.26):

$$
g_{\mu v}=g_{\mu \nu}^{(0)}+\delta g_{\mu v} .
$$

The background metric depends only on time, whereas the metric fluctuations $\delta g_{\mu \nu}$ depend on both space and time. Since the metric is a symmetric tensor, there are at first sight 10 fluctuating degrees of freedom in $\delta g_{\mu \nu}$.

There are four degrees of freedom which correspond to scalar metric fluctuations (the only four ways of constructing a metric from scalar functions):

$$
\delta g_{\mu v}=a^{2}\left(\begin{array}{cc}
2 \phi & -B_{, i} \\
-B_{, i} & 2\left(\psi \delta_{i j}-E_{, i j}\right)
\end{array}\right),
$$


where the four fluctuating degrees of freedom are denoted (following the notation of [34]) $\phi, B, E$, and $\psi$, a comma denotes the ordinary partial derivative (if we had included spatial curvature of the background metric, it would have been the covariant derivative with respect to the spatial metric), and $\delta_{i j}$ is the Kronecker symbol.

There are also four vector degrees of freedom of metric fluctuations, consisting of the four ways of constructing metric fluctuations from three vectors:

$$
\delta g_{\mu \nu}=a^{2}\left(\begin{array}{cc}
0 & -S_{i} \\
-S_{i} & F_{i, j}+F_{j, i}
\end{array}\right),
$$

where $S_{i}$ and $F_{i}$ are two divergence-less vectors (for a vector with non-vanishing divergence, the divergence contributes to the scalar gravitational fluctuation modes).

Finally, there are two tensor modes which correspond to the two polarization states of gravitational waves:

$$
\delta g_{\mu v}=-a^{2}\left(\begin{array}{cc}
0 & 0 \\
0 & h_{i j}
\end{array}\right),
$$

where $h_{i j}$ is trace-free and divergence-less

$$
h_{i}^{i}=h_{i j}^{j}=0 .
$$

Gravitational waves do not couple at linear order to the matter fluctuations. Vector fluctuations decay in an expanding background cosmology and hence are not usually cosmologically important. The most important fluctuations, at least in inflationary cosmology, are the scalar metric fluctuations, the fluctuations which couple to matter inhomogeneities and which are the relativistic generalization of the Newtonian perturbations considered in the previous section. Note that vector and tensor perturbations are important (see e.g. [48]) in topological defects models of structure formation and in bouncing cosmologies [49].

\subsubsection{Gauge Transformation}

The theory of cosmological perturbations is at first sight complicated by the issue of gauge invariance (at the final stage, however, we will see that we can make use of the gauge freedom to substantially simplify the theory). The coordinates $t, \mathbf{x}$ of space-time carry no independent physical meaning. They are just labels to designate points in the space-time manifold. By performing a small-amplitude transformation of the space-time coordinates (called "gauge transformation" in the following), we can easily introduce "fictitious" fluctuations in a homogeneous and isotropic Universe. These modes are "gauge artifacts".

We will in the following take an "active" view of gauge transformation. Let us consider two space-time manifolds, one of them a homogeneous and isotropic Universe $\mathscr{M}_{0}$, the other a physical Universe $\mathscr{M}$ with inhomogeneities. A choice of coordinates can be considered to be a mapping $\mathscr{D}$ between the manifolds $\mathscr{M}_{0}$ and $\mathscr{M}$. Let us consider a second mapping $\tilde{\mathscr{D}}$ which will take the same point (e.g. the origin of a fixed coordinate system) in $\mathscr{M}_{0}$ to a different point in $\mathscr{M}$. Using the inverse of these maps $\mathscr{D}$ and $\tilde{D}$, we can assign two different sets of coordinates to points in $\mathscr{M}$.

Consider now a physical quantity $Q$ (e.g. the Ricci scalar) on $\mathscr{M}$, and the corresponding physical quantity $Q^{(0)}$ on $\mathscr{M}_{0}$ Then, in the first coordinate system given by the mapping $\mathscr{D}$, the 
perturbation $\delta Q$ of $Q$ at the point $p \in \mathscr{M}$ is defined by

$$
\delta Q(p)=Q(p)-Q^{(0)}\left(\mathscr{D}^{-1}(p)\right) .
$$

Analogously, in the second coordinate system given by $\tilde{\mathscr{D}}$, the perturbation is defined by

$$
\tilde{\delta Q}(p)=Q(p)-Q^{(0)}\left(\tilde{\mathscr{D}}^{-1}(p)\right) .
$$

The difference

$$
\Delta Q(p)=\tilde{\delta Q}(p)-\delta Q(p)
$$

is obviously a gauge artifact and carries no physical significance.

Some of the degrees of freedom of metric perturbation introduced in the first subsection are gauge artifacts. To isolate these, we must study how coordinate transformations act on the metric. There are four independent gauge degrees of freedom corresponding to the coordinate transformation

$$
x^{\mu} \rightarrow \tilde{x}^{\mu}=x^{\mu}+\xi^{\mu} .
$$

The zero (time) component $\xi^{0}$ of $\xi^{\mu}$ leads to a scalar metric fluctuation. The spatial three vector $\xi^{i}$ can be decomposed as

$$
\xi^{i}=\xi_{t r}^{i}+\gamma^{i j} \xi_{, j}
$$

(where $\gamma^{i j}$ is the spatial background metric) into a transverse piece $\xi_{t r}^{i}$ which has two degrees of freedom which yield vector perturbations, and the second term (given by the gradient of a scalar $\xi$ ) which gives a scalar fluctuation. To summarize this paragraph, there are two scalar gauge modes given by $\xi^{0}$ and $\xi$, and two vector modes given by the transverse three vector $\xi_{t r}^{i}$. Thus, there remain two physical scalar and two vector fluctuation modes. The gravitational waves are gaugeinvariant.

Let us now focus on how the scalar gauge transformations (i.e. the transformations given by $\xi^{0}$ and $\xi$ ) act on the scalar metric fluctuation variables $\phi, B, E$, and $\psi$. An immediate calculation yields:

$$
\begin{aligned}
\tilde{\phi} & =\phi-\frac{a^{\prime}}{a} \xi^{0}-\left(\xi^{0}\right)^{\prime} \\
\tilde{B} & =B+\xi^{0}-\xi^{\prime} \\
\tilde{E} & =E-\xi \\
\tilde{\psi} & =\psi+\frac{a^{\prime}}{a} \xi^{0},
\end{aligned}
$$

where a prime indicates the derivative with respect to conformal time $\eta$.

There are two approaches to deal with the gauge ambiguities. The first is to fix a gauge, i.e. to pick conditions on the coordinates which completely eliminate the gauge freedom, the second is to work with a basis of gauge-invariant variables. If one wants to adopt the gauge-fixed approach, there are many different gauge choices. Note that the often used synchronous gauge determined by $\delta g^{0 \mu}=0$ does not totally fix the gauge. A convenient system which completely fixes the coordinates is the so-called longitudinal or conformal Newtonian gauge defined by $B=E=0$. This is the gauge we will use in the following. 
Note that the above analysis was strictly at linear order in perturbation theory. Beyond linear order, the structure of perturbation theory becomes much more involved. In fact, one can show [50] that the only fluctuation variables which are invariant under all coordinate transformations are perturbations of variables which are constant in the background space-time.

\subsubsection{Equations of Motion}

We begin with the Einstein equations

$$
G_{\mu v}=8 \pi G T_{\mu v},
$$

where $G_{\mu \nu}$ is the Einstein tensor associated with the space-time metric $g_{\mu \nu}$, and $T_{\mu \nu}$ is the energymomentum tensor of matter, insert the ansatz for metric and matter perturbed about a FRW background $\left(g_{\mu \nu}^{(0)}(\eta), \varphi^{(0)}(\eta)\right)$ :

$$
\begin{aligned}
g_{\mu v}(\mathbf{x}, \eta) & =g_{\mu v}^{(0)}(\eta)+\delta g_{\mu v}(\mathbf{x}, \eta) \\
\varphi(\mathbf{x}, \eta) & =\varphi_{0}(\eta)+\delta \varphi(\mathbf{x}, \eta)
\end{aligned}
$$

(where we have for simplicity replaced general matter by a scalar matter field $\varphi$ ) and expand to linear order in the fluctuating fields, obtaining the following equations:

$$
\delta G_{\mu \nu}=8 \pi G \delta T_{\mu \nu} .
$$

In the above, $\delta g_{\mu \nu}$ is the perturbation in the metric and $\delta \varphi$ is the fluctuation of the matter field $\varphi$.

Inserting the longitudinal gauge metric

$$
d s^{2}=a^{2}\left[(1+2 \phi) d \eta^{2}-(1-2 \psi) \gamma_{i j} d x^{i} d x^{j}\right]
$$

into the general perturbation equations (2.41) yields the following set of equations of motion:

$$
\begin{aligned}
-3 \mathscr{H}\left(\mathscr{H} \phi+\psi^{\prime}\right)+\nabla^{2} \psi & =4 \pi G a^{2} \delta T_{0}^{0} \\
\left(\mathscr{H} \phi+\psi^{\prime}\right)_{, i} & =4 \pi G a^{2} \delta T_{i}^{0} \\
{\left[\left(2 \mathscr{H}^{\prime}+\mathscr{H}^{2}\right) \phi+\mathscr{H} \phi^{\prime}+\psi^{\prime \prime}+2 \mathscr{H} \psi^{\prime}\right] \delta_{j}^{i} } & \\
+\frac{1}{2} \nabla^{2} D \delta_{j}^{i}-\frac{1}{2} \gamma^{i k} D_{, k j} & =-4 \pi G a^{2} \delta T_{j}^{i},
\end{aligned}
$$

where $D \equiv \phi-\psi$ and $\mathscr{H}=a^{\prime} / a$.

The first conclusion we can draw is that if no anisotropic stress is present in the matter at linear order in fluctuating fields, i.e. $\delta T_{j}^{i}=0$ for $i \neq j$, then the two metric fluctuation variables coincide:

$$
\phi=\psi .
$$

This will be the case in most simple cosmological models, e.g. in theories with matter described by a set of scalar fields with canonical form of the action, and in the case of a perfect fluid with no anisotropic stress.

Let us now restrict our attention to the case of matter described in terms of a single scalar field $\varphi$ (in which case the perturbations on large scales are automatically adiabatic) which can be expanded as

$$
\varphi(\mathbf{x}, \eta)=\varphi_{0}(\eta)+\delta \varphi(\mathbf{x}, \eta)
$$


in terms of background matter $\varphi_{0}$ and matter fluctuation $\delta \varphi(\mathbf{x}, \eta)$. Then, in longitudinal gauge, (2.43) reduce to the following set of equations of motion

$$
\begin{aligned}
\nabla^{2} \phi-3 \mathscr{H} \phi^{\prime}-\left(\mathscr{H}^{\prime}+2 \mathscr{H}^{2}\right) \phi & =4 \pi G\left(\varphi_{0}^{\prime} \delta \varphi^{\prime}+V^{\prime} a^{2} \delta \varphi\right) \\
\phi^{\prime}+\mathscr{H} \phi & =4 \pi G \varphi_{0}^{\prime} \delta \varphi \\
\phi^{\prime \prime}+3 \mathscr{H} \phi^{\prime}+\left(\mathscr{H}^{\prime}+2 \mathscr{H}^{2}\right) \phi & =4 \pi G\left(\varphi_{0}^{\prime} \delta \varphi^{\prime}-V^{\prime} a^{2} \delta \varphi\right),
\end{aligned}
$$

where $V^{\prime}$ denotes the derivative of $V$ with respect to $\varphi$. These equations can be combined to give the following second order differential equation for the relativistic potential $\phi$ :

$$
\phi^{\prime \prime}+2\left(\mathscr{H}-\frac{\varphi_{0}^{\prime \prime}}{\varphi_{0}^{\prime}}\right) \phi^{\prime}-\nabla^{2} \phi+2\left(\mathscr{H}^{\prime}-\mathscr{H} \frac{\varphi_{0}^{\prime \prime}}{\varphi_{0}^{\prime}}\right) \phi=0 .
$$

This is the final result for the classical evolution of cosmological fluctuations. First of all, we note the similarities with the equation (2.10) obtained in the Newtonian theory. The final term in (2.47) is the force due to gravity leading to the instability, the second to last term is the pressure force leading to oscillations (relativistic since we are considering matter to be a relativistic field), and the second term represents the Hubble friction. For each wavenumber there are two fundamental solutions. On small scales $(k>H)$, the solutions correspond to damped oscillations, on large scales $(k<H)$ the oscillations freeze out and the dynamics is governed by the gravitational force competing with the Hubble friction term. Note, in particular, how the Hubble radius naturally emerges as the scale where the nature of the fluctuating modes changes from oscillatory to frozen.

Considering the equation in a bit more detail, we observe that if the equation of state of the background is independent of time (which will be the case if $\mathscr{H}^{\prime}=\varphi_{0}^{\prime \prime}=0$ ), then in an expanding background, the dominant mode of (2.47) is constant, and the sub-dominant mode decays. If the equation of state is not constant, then the dominant mode is not constant in time. Specifically, at the end of inflation $\mathscr{H}^{\prime}<0$, and this leads to a growth of $\phi$ (see the following subsection). In contrast, in a contracting phase the dominant mode of $\phi$ is growing.

To study the quantitative implications of the equation of motion (2.47), it is convenient to introduce $[51,52]$ the variable $\zeta$ (which, up to a correction term of the order $\nabla^{2} \phi$ which is unimportant for large-scale fluctuations, is equal to the curvature perturbation $\mathscr{R}$ in comoving gauge [53]) by

$$
\zeta \equiv \phi+\frac{2}{3} \frac{\left(H^{-1} \dot{\phi}+\phi\right)}{1+w}
$$

where

$$
w=\frac{p}{\rho}
$$

characterizes the equation of state of matter. In terms of $\zeta$, the equation of motion (2.47) takes on the form

$$
\frac{3}{2} \dot{\zeta} H(1+w)=\mathscr{O}\left(\nabla^{2} \phi\right)
$$

On large scales, the right hand side of the equation is negligible, which leads to the conclusion that large-scale cosmological fluctuations satisfy

$$
\dot{\zeta}(1+w)=0 .
$$


This implies that $\zeta$ is constant except possibly if $1+w=0$ at some point in time during the cosmological evolution (which occurs during reheating in inflationary cosmology if the inflaton field undergoes oscillations [54]). In single matter field models it is indeed possible to show that $\dot{\zeta}=0$ on super-Hubble scales independent of assumptions on the equation of state [55, 56]. This "conservation law" makes it easy to relate initial fluctuations to final fluctuations in inflationary cosmology, as will be illustrated in the following subsection.

In the presence of non-adiabatic fluctuations (entropy fluctuations) there is a source term on the righ-hand side of (2.51) which is proportional to the amplitude of the entropy fluctuations. Thus, as already seen in the case of the Newtonian theory of cosmological fluctuations, non-adiabatic fluctuations induce a growing adiabatic mode (see $[57,58]$ for discussions of the consequences in double field inflationary models).

\subsection{Quantum Theory}

\subsubsection{Overview}

As we have seen in the first section, in many models of the very early Universe, in particular in inflationary cosmology, in string gas cosmology and in the matter bounce scenario, primordial inhomogeneities are generated in an initial phase on sub-Hubble scales. The wavelength is then stretched relative to the Hubble radius, becomes larger than the Hubble radius at some time and then propagates on super-Hubble scales until re-entering at late cosmological times. In a majority of the current structure formation scenarios (string gas cosmology is an exception in this respect), fluctuations are assumed to emerge as quantum vacuum perturbations. Hence, to describe the generation and evolution of the inhomogeneities a quantum treatment is required.

In the context of a Universe with a de Sitter phase, the quantum origin of cosmological fluctuations was first discussed in [6] and also [7, 5] for earlier ideas. In particular, Mukhanov [6] and Press [7] realized that in an exponentially expanding background, the curvature fluctuations would be scale-invariant, and Mukhanov provided a quantitative calculation which also yielded the logarithmic deviation from exact scale-invariance.

The role of the Hubble radius has already been mentioned repeatedly in these lectures. In particular, in the previous subsection we saw that the Hubble radius separates scales on which fluctuations oscillate (sub-Hubble scales) from those where they are frozen in (super-Hubble scales). Another way to see the role of the Hubble radius is to consider the equation of a free scalar matter field $\varphi$ on an unperturbed expanding background:

$$
\ddot{\varphi}+3 H \dot{\varphi}-\frac{\nabla^{2}}{a^{2}} \varphi=0 .
$$

The second term on the left hand side of this equation leads to damping of $\varphi$ with a characteristic decay rate given by $H$. As a consequence, in the absence of the spatial gradient term, $\dot{\varphi}$ would be of the order of magnitude $H \varphi$. Thus, comparing the second and the third terms on the left hand side, we immediately see that the microscopic (spatial gradient) term dominates on length scales smaller than the Hubble radius, leading to oscillatory motion, whereas this term is negligible on scales larger than the Hubble radius, and the evolution of $\varphi$ is determined primarily by gravity. Note that in general cosmological models the Hubble radius is much smaller than the horizon (the forward light cone calculated from the initial time). In an inflationary universe, the horizon is 
larger by a factor of at least $\exp (N)$, where $N$ is the number of e-foldings of inflation, and the lower bound is taken on if the Hubble radius and horizon coincide until inflation begins. It is very important to realize this difference, a difference which is obscured in most articles on cosmology in which the term "horizon" is used when "Hubble radius" is meant. Note, in particular, that the homogeneous inflaton field contains causal information on super-Hubble but sub-horizon scales. Hence, it is completely consistent with causality [54] to have a microphysical process related to the background scalar matter field lead to exponential amplification of the amplitude of fluctuations during reheating on such scales, as it does in models in which entropy perturbations are present and not suppressed during inflation $[57,58]$. Note that also in string gas cosmology and in the matter bounce scenario the Hubble radius and horizon are completely different.

There are general relativistic conservation laws [59] which imply that adiabatic fluctuations produced locally must be Poisson-statistic suppressed on scales larger than the Hubble radius. For example, fluctuations produced by the formation of topological defects at a phase transition in the early universe are initially isocurvature (entropy) in nature (see e.g. [60] for a discussion). Via the source term in the equation of motion (2.4), a growing adiabatic mode is induced, but at any fixed time the spectrum of the curvature fluctuation on scales larger than the Hubble radius has index $n=4$ (Poisson). A similar conclusion applies to models [61, 62] of modulated reheating (see [63] for a nice discussion), and to models in which moduli fields obtain masses after some symmetry breaking, their quantum fluctuations then inducing cosmological perturbations. A prototypical example is given by axion fluctuations in an inflationary universe (see e.g. [64] and references therein).

To understand the generation and evolution of fluctuations in current models of the very early Universe, we need both Quantum Mechanics and General Relativity, i.e. quantum gravity. At first sight, we are thus faced with an intractable problem, since the theory of quantum gravity is not yet established. We are saved by the fact that today on large cosmological scales the fractional amplitude of the fluctuations is smaller than 1 . Since gravity is a purely attractive force, the fluctuations had to have been - at least in the context of an eternally expanding background cosmology - very small in the early Universe. Thus, a linearized analysis of the fluctuations (about a classical cosmological background) is self-consistent.

From the classical theory of cosmological perturbations discussed in the previous section, we know that the analysis of scalar metric inhomogeneities can be reduced - after extracting gauge artifacts - to the study of the evolution of a single fluctuating variable. Thus, we conclude that the quantum theory of cosmological perturbations must be reducible to the quantum theory of a single free scalar field which we will denote by $v$. Since the background in which this scalar field evolves is time-dependent, the mass of $v$ will be time-dependent. The time-dependence of the mass will lead to quantum particle production over time if we start the evolution in the vacuum state for $v$. As we will see, this quantum particle production corresponds to the development and growth of the cosmological fluctuations. Thus, the quantum theory of cosmological fluctuations provides a consistent framework to study both the generation and the evolution of metric perturbations. The following analysis is based on Part II of [34].

\subsubsection{Outline of the Analysis}

In order to obtain the action for linearized cosmological perturbations, we expand the action 
to quadratic order in the fluctuating degrees of freedom. The linear terms cancel because the background is taken to satisfy the background equations of motion.

We begin with the Einstein-Hilbert action for gravity and the action of a scalar matter field (for the more complicated case of general hydrodynamical fluctuations the reader is referred to [34])

$$
S=\int d^{4} x \sqrt{-g}\left[-\frac{1}{16 \pi G} R+\frac{1}{2} \partial_{\mu} \varphi \partial^{\mu} \varphi-V(\varphi)\right],
$$

where $R$ is the Ricci curvature scalar.

The simplest way to proceed is to work in longitudinal gauge, in which the metric and matter take the form (assuming no anisotropic stress)

$$
\begin{aligned}
d s^{2} & =a^{2}(\eta)\left[(1+2 \phi(\eta, \mathbf{x})) d \eta^{2}-(1-2 \phi(t, \mathbf{x})) d \mathbf{x}^{2}\right] \\
\varphi(\eta, \mathbf{x}) & =\varphi_{0}(\eta)+\delta \varphi(\eta, \mathbf{x}) .
\end{aligned}
$$

The two fluctuation variables $\phi$ and $\varphi$ must be linked by the Einstein constraint equations since there cannot be matter fluctuations without induced metric fluctuations.

The two nontrivial tasks of the lengthy [34] computation of the quadratic piece of the action is to find out what combination of $\varphi$ and $\phi$ gives the variable $v$ in terms of which the action has canonical kinetic term, and what the form of the time-dependent mass is. This calculation involves inserting the ansatz (2.54) into the action (2.53), expanding the result to second order in the fluctuating fields, making use of the background and of the constraint equations, and dropping total derivative terms from the action. In the context of scalar field matter, the quantum theory of cosmological fluctuations was developed by Mukhanov [65, 66] and Sasaki [67]. The result is the following contribution $S^{(2)}$ to the action quadratic in the perturbations:

$$
S^{(2)}=\frac{1}{2} \int d^{4} x\left[v^{2}-v_{, i} v_{, i}+\frac{z^{\prime \prime}}{z} v^{2}\right],
$$

where the canonical variable $v$ (the "Sasaki-Mukhanov variable" introduced in [66] - see also [68]) is given by

$$
v=a\left[\delta \varphi+\frac{\varphi_{0}^{\prime}}{\mathscr{H}} \phi\right]
$$

with $\mathscr{H}=a^{\prime} / a$, and where

$$
z=\frac{a \varphi_{0}^{\prime}}{\mathscr{H}} .
$$

As long as the equation of state does not change over time)

$$
z(\eta) \sim a(\eta) .
$$

Note that the variable $v$ is related to the curvature perturbation $\mathscr{R}$ in comoving coordinates introduced in [53] and closely related to the variable $\zeta$ used in [51, 52]:

$$
v=z \mathscr{R} .
$$

The equation of motion which follows from the action (2.55) is (in momentum space)

$$
v_{k}^{\prime \prime}+k^{2} v_{k}-\frac{z^{\prime \prime}}{z} v_{k}=0,
$$


where $v_{k}$ is the k'th Fourier mode of $v$. The mass term in the above equation is in general given by the Hubble scale. Thus, it immediately follows that on small length scales, i.e. for $k>k_{H}$, the solutions for $v_{k}$ are constant amplitude oscillations. These oscillations freeze out at Hubble radius crossing, i.e. when $k=k_{H}$. On longer scales $\left(k \ll k_{H}\right)$, there is a mode of $v_{k}$ which scales as $z$. This mode is the dominant one in an expanding universe, but not in a contracting one.

Given the action (2.55), the quantization of the cosmological perturbations can be performed by canonical quantization (in the same way that a scalar matter field on a fixed cosmological background is quantized [69]).

The final step in the quantum theory of cosmological perturbations is to specify an initial state. Since in inflationary cosmology all pre-existing classical fluctuations are red-shifted by the accelerated expansion of space, one usually assumes (we will return to a criticism of this point when discussing the trans-Planckian problem of inflationary cosmology) that the field $v$ starts out at the initial time $t_{i}$ mode by mode in its vacuum state. Two questions immediately emerge: what is the initial time $t_{i}$, and which of the many possible vacuum states should be chosen. It is usually assumed that since the fluctuations only oscillate on sub-Hubble scales, the choice of the initial time is not important, as long as it is earlier than the time when scales of cosmological interest today cross the Hubble radius during the inflationary phase. The state is usually taken to be the Bunch-Davies vacuum (see e.g. [69]), since this state is empty of particles at $t_{i}$ in the coordinate frame determined by the FRW coordinates Thus, we choose the initial conditions

$$
\begin{gathered}
v_{k}\left(\eta_{i}\right)=\frac{1}{\sqrt{2 k}} \\
v_{k}^{\prime}\left(\eta_{i}\right)=\frac{\sqrt{k}}{\sqrt{2}}
\end{gathered}
$$

where $\eta_{i}$ is the conformal time corresponding to the physical time $t_{i}$.

Returning to the case of an expanding universe, the scaling

$$
v_{k} \sim z \sim a
$$

implies that the wave function of the quantum variable $v_{k}$ which performs quantum vacuum fluctuations on sub-Hubble scales, stops oscillating on super-Hubble scales and instead is squeezed (the amplitude increases in configuration space but decreases in momentum space). This squeezing corresponds to quantum particle production. It is also one of the two conditions which are required for the classicalization of the fluctuations. The second condition is decoherence which is induced by the non-linearities in the dynamical system which are inevitable since the Einstein action leads to highly nonlinear equatiions (see [70] for a recent discussion of this point, and [71] for related work).

Note that the squeezing of cosmological fluctuations on super-Hubble scales occurs in all models, in particular in string gas cosmology and in the bouncing universe scenario since also in these scenarios perturbations propagate on super-Hubble scales for a long period of time. In a contracting phase, the dominant mode of $v_{k}$ on super-Hubble scales is not the one given in (2.62) (which in this case is a decaying mode), but the second mode which scales as $z^{-p}$ with an exponent $p$ which is positive and whose exact value depends on the background equation of state.

Applications of this theory in inflationary cosmology, in the matter bounce scenario and in string gas cosmology will be considered in the respective sections of these lecture notes. 


\subsubsection{Quantum Theory of Gravitational Waves}

The quantization of gravitational waves parallels the quantization of scalar metric fluctuations, but is more simple because there are no gauge ambiguities. Note that at the level of linear fluctuations, scalar metric fluctuations and gravitational waves are independent. Both can be quantized on the same cosmological background determined by the background scale factor and the background matter. However, in contrast to the case of scalar metric fluctuations, the tensor modes are also present in pure gravity (i.e. in the absence of matter).

Starting point is the action (2.53). Into this action we insert the metric which corresponds to a classical cosmological background plus tensor metric fluctuations:

$$
d s^{2}=a^{2}(\eta)\left[d \eta^{2}-\left(\delta_{i j}+h_{i j}\right) d x^{i} d x^{j}\right],
$$

where the second rank tensor $h_{i j}(\eta, \mathbf{x})$ represents the gravitational waves, and in turn can be decomposed as

$$
h_{i j}(\eta, \mathbf{x})=h_{+}(\eta, \mathbf{x}) e_{i j}^{+}+h_{x}(\eta, \mathbf{x}) e_{i j}^{x}
$$

into the two polarization states. Here, $e_{i j}^{+}$and $e_{i j}^{x}$ are two fixed polarization tensors, and $h_{+}$and $h_{x}$ are the two coefficient functions.

To quadratic order in the fluctuating fields, the action consists of separate terms involving $h_{+}$ and $h_{x}$. Each term is of the form

$$
S^{(2)}=\int d^{4} x \frac{a^{2}}{2}\left[h^{\prime 2}-(\nabla h)^{2}\right]
$$

leading to the equation of motion

$$
h_{k}^{\prime \prime}+2 \frac{a^{\prime}}{a} h_{k}^{\prime}+k^{2} h_{k}=0 .
$$

The variable in terms of which the action (2.65) has canonical kinetic term is

$$
\mu_{k} \equiv a h_{k},
$$

and its equation of motion is

$$
\mu_{k}^{\prime \prime}+\left(k^{2}-\frac{a^{\prime \prime}}{a}\right) \mu_{k}=0 .
$$

This equation is very similar to the corresponding equation (2.60) for scalar gravitational inhomogeneities, except that in the mass term the scale factor $a(\eta)$ replaces $z(\eta)$, which leads to a very different evolution of scalar and tensor modes during the reheating phase in inflationary cosmology during which the equation of state of the background matter changes dramatically.

Based on the above discussion we have the following theory for the generation and evolution of gravitational waves in an accelerating Universe (first developed by Grishchuk [72]): waves exist as quantum vacuum fluctuations at the initial time on all scales. They oscillate until the length scale crosses the Hubble radius. At that point, the oscillations freeze out and the quantum state of gravitational waves begins to be squeezed in the sense that

$$
\mu_{k}(\eta) \sim a(\eta)
$$

which, from (2.67) corresponds to constant amplitude of $h_{k}$. The squeezing of the vacuum state leads to the emergence of classical properties of this state, as in the case of scalar metric fluctuations. 


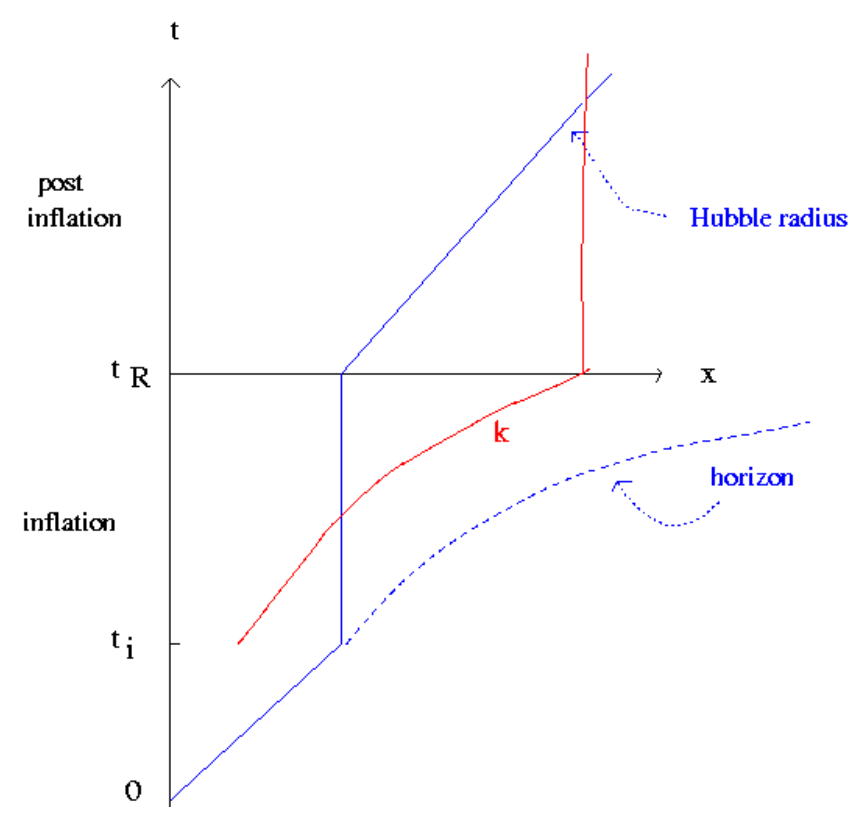

Figure 9: Space-time diagram (sketch) showing the evolution of scales in inflationary cosmology. The vertical axis is time, and the period of inflation lasts between $t_{i}$ and $t_{R}$, and is followed by the radiationdominated phase of standard big bang cosmology. During exponential inflation, the Hubble radius $H^{-1}$ is constant in physical spatial coordinates (the horizontal axis), whereas it increases linearly in time after $t_{R}$. The physical length corresponding to a fixed comoving length scale labelled by its wavenumber $k$ increases exponentially during inflation but increases less fast than the Hubble radius (namely as $t^{1 / 2}$ ), after inflation.

\section{Inflationary Cosmology}

\subsection{Mechanism of Inflation}

The idea of inflationary cosmology is to assume that there was a period in the very early Universe during which the scale factor was accelerating, i.e. $\ddot{a}>0$. This implies that the Hubble radius was shrinking in comoving coordinates, or, equivalently, that fixed comoving scales were "exiting" the Hubble radius. In the simplest models of inflation, the scale factor increases nearly exponentially. As illustrated in Figure 9, the basic geometry of inflationary cosmology provides a solution of the fluctuation problem. As long as the phase of inflation is sufficiently long, all length scales within our present Hubble radius today originate at the beginning of inflation with a wavelength smaller than the Hubble radius at that time. Thus, it is possible to create perturbations locally using physics obeying the laws of special relativity (in particular causality). As will be discussed later, it is quantum vacuum fluctuations of matter fields and their associated curvature perturbations which are responsible for the structure we observe today.

Postulating a phase of inflation in the very early universe solves the horizon problem of the SBB, namely it explains why the causal horizon at the time $t_{\text {rec }}$ when photons last scatter is larger than the radius of the past light cone at $t_{r e c}$, the part of the last scattering surface which is visible today in CMB experiments. Inflation also explains the near flatness of the universe: in a decelerating universe spatial flatness is an unstable fixed point of the dynamics, whereas in an accelerating universe it becomes an attractor. Another important aspect of the inflationary solution of the flat- 
ness problem is that inflation exponentially increases the volume of space. Without inflation, it is not possible that a Planck scale universe at the Planck time evolves into a sufficiently large universe today.

\subsection{Fluctuations in Inflationary Cosmology}

We will now use the quantum theory of cosmological perturbations developed in the previous section to calculate the spectrum of curvature fluctuations in inflationary cosmology. The starting point are quantum vacuum initial conditions for the canonical fluctuation variable $v_{k}$ :

$$
v_{k}\left(\eta_{i}\right)=\frac{1}{\sqrt{2 k}}
$$

for all $k$ for which the wavelength is smaller than the Hubble radius at the initial time $t_{i}$.

The amplitude remains unchanged until the modes exit the Hubble radius at the respective times $t_{H}(k)$ given by

$$
a^{-1}\left(t_{H}(k)\right) k=H .
$$

We need to compute the power spectrum $\mathscr{P}_{\mathscr{R}}(k)$ of the curvature fluctuation $\mathscr{R}$ defined in (2.59) at some late time $t$ when the modes are super-Hubble. We first relate the power spectrum via the growth rate (2.62) of $v$ on super-Hubble scales to the power spectrum at the time $t_{H}(k)$ and then use the constancy of the amplitude of $v$ on sub-Hubble scales to relate it to the initial conditions (3.1). Thus

$$
\begin{aligned}
\mathscr{P}_{\mathscr{R}}(k, t) \equiv k^{3} \mathscr{R}_{k}^{2}(t) & =k^{3} z^{-2}(t)\left|v_{k}(t)\right|^{2} \\
& =k^{3} z^{-2}(t)\left(\frac{a(t)}{a\left(t_{H}(k)\right)}\right)^{2}\left|v_{k}\left(t_{H}(k)\right)\right|^{2} \\
& =k^{3} z^{-2}\left(t_{H}(k)\right)\left|v_{k}\left(t_{H}(k)\right)\right|^{2} \\
& \sim k^{3}\left(\frac{a(t)}{z(t)}\right)^{2} a^{-2}\left(t_{H}(k)\right)\left|v_{k}\left(t_{i}\right)\right|^{2},
\end{aligned}
$$

where in the final step we have used (2.58) and the constancy of the amplitude of $v$ on sub-Hubble scales. Making use of the condition (3.2) for Hubble radius crossing, and of the initial conditions (3.1), we immediately see that

$$
\mathscr{P}_{\mathscr{R}}(k, t) \sim\left(\frac{a(t)}{z(t)}\right)^{2} k^{3} k^{-2} k^{-1} H^{2}
$$

and that thus a scale invariant power spectrum with amplitude proportional to $H^{2}$ results, in agreement with what was argued on heuristic grounds in the overview of inflation in the the first section. To obtain the precise amplitude, we need to make use of the relation between $z$ and $a$. We obtain

$$
\mathscr{P}_{\mathscr{R}}(k, t) \sim \frac{H^{4}}{\dot{\varphi}_{0}^{2}}
$$

which for any given value of $k$ is to be evaluated at the time $t_{H}(k)$ (before the end of inflation). For a scalar field potential (see following subsection)

$$
V(\varphi)=\lambda \varphi^{4}
$$

the resulting amplitude in (3.5) is $\lambda$. Thus, in order to obtain the observed value of the power spectrum of the order of $10^{-10}$, the coupling constant $\lambda$ must be tuned to a very small value. 


\subsection{Models of Inflation}

Let us now consider how it is possible to obtain a phase of cosmological inflation. We will assume that space-time is described using the equations of General Relativity ${ }^{4}$. In this case, the dynamics of the scale factor $a(t)$ is determined by the Friedmann-Robertson-Walker (FRW) equations

$$
\left(\frac{\dot{a}}{a}\right)^{2}=8 \pi G \rho
$$

and

$$
\frac{\ddot{a}}{a}=-\frac{4 \pi G}{3}(\rho+3 p)
$$

where for simplicity we have omitted the contributions of spatial curvature (since spatial curvature is diluted during inflation) and of the cosmological constant (since any small cosmological constant which might be present today has no effect in the early Universe since the associated energy density does not increase when going into the past). From (3.8) it is clear that in order to obtain an accelerating universe, matter with sufficiently negative pressure

$$
p<-\frac{1}{3} \rho
$$

is required. Exponential inflation is obtained for $p=-\rho$.

Conventional perfect fluids have positive semi-definite pressure and thus cannot yield inflation. Quantum field theory can come to the rescue. We know that a description of matter in terms of classical perfect fluids must break down at early times. An improved description of matter will be given in terms of quantum fields. Matter which we observe today consists of spin 1/2 and spin 1 fields. Such fields cannot yield inflation in the context of the renormalizable quantum field theory framework. The existence of scalar matter fields is postulated to explain the generation of mass via spontaneous symmetry breaking. Scalar matter fields (denoted by $\varphi$ ) are special in that they allow - at the level of a renormalizable action - the presence of a potential energy term $V(\varphi)$. The energy density and pressure of a scalar field $\varphi$ with canonically normalized action ${ }^{5}$

$$
\mathscr{L}=\sqrt{-g}\left[\frac{1}{2} \partial_{\mu} \varphi \partial^{\mu} \varphi-V(\varphi)\right]
$$

(where Greek indices are space-time indices and $g$ is the determinant of the metric) are given by

$$
\begin{aligned}
\rho & =\frac{1}{2}(\dot{\varphi})^{2}+\frac{1}{2} a^{-2}(\nabla \varphi)^{2}+V(\varphi) \\
p & =\frac{1}{2}(\dot{\varphi})^{2}-\frac{1}{6} a^{-2}(\nabla \varphi)^{2}-V(\varphi) .
\end{aligned}
$$

Thus, it is possible to obtain an almost exponentially expanding universe provided the scalar field configuration ${ }^{6}$ satisfies

$$
\begin{aligned}
\frac{1}{2}\left(\nabla_{p} \varphi\right)^{2} & \ll V(\varphi), \\
\frac{1}{2}(\dot{\varphi})^{2} & \ll V(\varphi) .
\end{aligned}
$$

\footnotetext{
${ }^{4}$ Note, however, that the first model of exponential expansion of space [4] made use of a higher derivative gravitational action.

${ }^{5}$ See $[73,74]$ for discussions of fields with non-canonical kinetic terms.

${ }^{6}$ The scalar field yielding inflation is called the inflaton.
} 
In the above, $\nabla_{p} \equiv a^{-1} \nabla$ is the gradient with respect to physical as opposed to comoving coordinates. Since spatial gradients redshift as the universe expands, the first condition will (for single scalar field models) always be satisfied if it is satisfied at the initial time ${ }^{7}$. It is the second condition which is harder to satisfy. In particular, this condition is in general not preserved in time even it is initially satisfied.

In his original model [2], Guth assumed that inflation was generated by a scalar field $\varphi$ sitting in a false vacuum with positive vacuum energy. Hence, the conditions (3.12) and (3.13) are automatically satisfied. Inflation ends when $\varphi$ tunnels to its true vacuum state with (by assumption) vanishing potential energy. However, as Guth realized himself, this model is plagued by a "graceful exit" problem: the nucleation produces a bubble of true vacuum whose initial size is microscopic [76] (see also [77] for a review of field theory methods which are useful in inflationary cosmology). After inflation, this bubble will expand at the speed of light, but it is too small to explain the observed size of the universe. Since space between the bubbles expands exponentially, the probability of bubble percolation is negligible, at least in Einstein gravity. For this reason, the focus in inflationary model building shifted to the "slow-roll" paradigm [78].

It is sufficient to obtain a period of cosmological inflation that the slow-roll conditions for $\varphi$ are satisfied. Recall that the equation of motion for a homogeneous scalar field in a cosmological space-time is (as follows from (3.10)) is

$$
\ddot{\varphi}+3 H \dot{\varphi}=-V^{\prime}(\varphi)
$$

where a prime indicates the derivative with respect to $\varphi$. In order that the scalar field roll slowly, it is necessary that

$$
\ddot{\varphi} \ll 3 H \dot{\varphi}
$$

such that the first term in the scalar field equation of motion (3.14) is negligible. In this case, the condition (3.13) becomes

$$
\left(\frac{V^{\prime}}{V}\right)^{2} \ll 48 \pi G
$$

and (3.15) becomes

$$
\frac{V^{\prime \prime}}{V} \ll 24 \pi G
$$

There are many models of scalar field-driven slow-roll inflation. Many of them can be divided into three groups: small-field inflation, large-field inflation and hybrid inflation. Small-field inflationary models are based on ideas from spontaneous symmetry breaking in particle physics. We take the scalar field to have a potential of the form

$$
V(\varphi)=\frac{1}{4} \lambda\left(\varphi^{2}-\sigma^{2}\right)^{2},
$$

where $\sigma$ can be interpreted as a symmetry breaking scale, and $\lambda$ is a dimensionless coupling constant. The hope of initial small-field models ("new inflation" [78]) was that the scalar field would begin rolling close to its symmetric point $\varphi=0$, where thermal equilibrium initial conditions would

\footnotetext{
${ }^{7}$ In fact, careful studies [75] show that since the gradients decrease even in a non-inflationary backgrounds, they can become subdominant even if they are not initially subdominant.
} 
localize it in the early universe. At sufficiently high temperatures, $\varphi=0$ is a stable ground state of the one-loop finite temperature effective potential $V_{T}(\varphi)$ (see e.g. [77] for a review). Once the temperature drops to a value smaller than the critical temperature $T_{c}, \varphi=0$ turns into an unstable local maximum of $V_{T}(\varphi)$, and $\varphi$ is free to roll towards a ground state of the zero temperature potential (3.18). The direction of the initial rolling is triggered by quantum fluctuations. The reader can easily check that for the potential (3.18) the slow-roll conditions cannot be satisfied if $\sigma \ll m_{p l}$, where $m_{p l}$ is the Planck mass which is related to $G$. If the potential is modified to a Coleman-Weinberg [79] form

$$
V(\varphi)=\frac{\lambda}{4} \varphi^{4}\left[\ln \frac{|\varphi|}{v}-\frac{1}{4}\right]+\frac{1}{16} \lambda v^{4}
$$

(where $v$ denotes the value of the minimum of the potential) then the slow-roll conditions can be satisfied. However, this corresponds to a severe fine-tuning of the shape of the potential. A further problem for most small-field models of inflation (see e.g. [80] for a review) is that in order to end up close to the slow-roll trajectory, the initial field velocity must be constrained to be very small. This initial condition problem of small-field models of inflation effects a number of recently proposed brane inflation scenarios, see e.g. [81] for a discussion.

There is another reason for abandoning small-field inflation models: in order to obtain a sufficiently small amplitude of density fluctuations, the interaction coefficients of $\varphi$ must be very small. This makes it inconsistent to assume that $\varphi$ started out in thermal equilibrium [82]. In the absence of thermal equilibrium, the phase space of initial conditions is much larger for large values of $\varphi$.

This brings us to the discussion of large-field inflation models, initially proposed in [83] under the name "chaotic inflation". The simplest example is provided by a massive scalar field with potential

$$
V(\varphi)=\frac{1}{2} m^{2} \varphi^{2}
$$

where $m$ is the mass. It is assumed that the scalar field rolls towards the origin from large values of $|\varphi|$. It is a simple exercise for the reader to verify that the slow-roll conditions (3.16) and (3.17) are satisfied provided

$$
|\varphi|>\frac{1}{\sqrt{12 \pi}} m_{p l} .
$$

Values of $|\varphi|$ comparable or greater than $m_{p l}$ are also required in other realizations of large-field inflation. Hence, one may worry whether such a toy model can consistently be embedded in a realistic particle physics model, e.g. supergravity. In many such models $V(\varphi)$ receives supergravityinduced correction terms which destroy the flatness of the potential for $|\varphi|>m_{p l}$. As can be seen by applying the formulas of the previous subsection to the potential (3.20), a value of $m \sim 10^{13} \mathrm{GeV}$ is required in order to obtain the observed amplitude of density fluctuations. Hence, the configuration space range with $|\varphi|>m_{p l}$ but $V(\varphi)<m_{p l}^{4}$ dominates the measure of field values. It can also be verified that the slow-roll trajectory is a local attractor in field initial condition space [75], even including metric fluctuations at the perturbative level [84].

With two scalar fields it is possible to construct a class of models which combine some of the nice features of large-field inflation (large phase space of initial conditions yielding inflation) and of small-field inflation (better contact with conventional particle physics). These are models of hybrid inflation [85]. To give a prototypical example, consider two scalar fields $\varphi$ and $\chi$ with a 
potential

$$
V(\varphi, \chi)=\frac{1}{4} \lambda_{\chi}\left(\chi^{2}-\sigma^{2}\right)^{2}+\frac{1}{2} m^{2} \varphi^{2}-\frac{1}{2} g^{2} \varphi^{2} \chi^{2} .
$$

In the absence of thermal equilibrium, it is natural to assume that $|\varphi|$ begins at large values, values for which the effective mass of $\chi$ is positive and hence $\chi$ begins at $\chi=0$. The parameters in the potential (3.22) are now chosen such that $\varphi$ is slowly rolling for values of $|\varphi|$ somewhat smaller than $m_{p l}$, but that the potential energy for these field values is dominated by the first term on the right-hand side of (3.22). The reader can easily verify that for this model it is no longer required to have values of $|\varphi|$ greater than $m_{p l}$ in order to obtain slow-rolling ${ }^{8}$ The field $\varphi$ is slowly rolling whereas the potential energy is determined by the contribution from $\chi$. Once $|\varphi|$ drops to the value

$$
\left|\varphi_{c}\right|=\frac{\sqrt{\lambda_{\chi}}}{g} \sigma
$$

the configuration $\chi=0$ becomes unstable and decays to its ground state $|\chi|=\sigma$, yielding a graceful exit from inflation. Since in this example the ground state of $\chi$ is not unique, there is the possibility of the formation of topological defects at the end of inflation (see [86, 87, 88] for reviews of topological defects in cosmology).

\subsection{Reheating in Inflationary Cosmology}

During the inflationary period the energy density of matter becomes completely dominated by the energy of the inflaton field. The density of regular matter (the kind of matter which is observed today) is exponentially diluted. An essential part of any inflationary model is the mechanism by which the energy density is transferred to regular matter at the end of the period of inflation. This is the so-called "reheating" process. It typically begins with a non-perturbative decay process called "preheating". We will give a brief overview of reheating in this subsection (for an in-depth recent review the reader is referred to [89].

\subsubsection{Preheating}

After the slow-roll conditions break down, the period of inflation ends, and the inflaton $\varphi$ begins to oscillate around its ground state. Due to couplings of $\varphi$ to other matter fields, the energy of the universe, which at the end of the period of inflation is stored completely in $\varphi$, gets transferred to the matter fields of the particle physics Standard Model. Initially, the energy transfer was described perturbatively [90]. Later, it was realized [91, 92, 93, 94] that through a parametric resonance instability, particles are very rapidly produced, leading to a fast energy transfer ("preheating"). The quanta later thermalize, and thereafter the universe evolves as described by SBB cosmology.

Let us give a brief overview of the theory of reheating in inflationary cosmology. We assume that the inflaton $\varphi$ is coupled to another scalar field $\chi$ and take the interaction Lagrangian to be

$$
\mathscr{L}_{\text {int }}=-\frac{1}{2} g^{2} \varphi^{2} \chi^{2}
$$

where $g$ is a dimensionless coupling constant.

\footnotetext{
${ }^{8}$ Note that the slow-roll conditions (3.16) and (3.17) were derived assuming that $H$ is given by the contribution of $\varphi$ to $V$ which is not the case here.
} 
In the initial perturbative analysis of reheating [90] the decay of the coherently oscillating inflaton field $\varphi$ is treated perturbatively. The interaction rate $\Gamma$ of processes in which two $\varphi$ quanta interact to produce a pair of $\chi$ particles is taken as the decay rate of the inflaton. The inflaton dynamics is modeled via an effective equation

$$
\ddot{\varphi}+3 H \dot{\varphi}+\Gamma \dot{\varphi}=-V^{\prime}(\varphi) .
$$

For small coupling constant, the interaction rate $\Gamma$ is typically much smaller than the Hubble parameter at the end of inflation. Thus, at the beginning of the phase of inflaton oscillations, the energy loss into particles is initially negligible compared to the energy loss due to the expansion of space. It is only once the Hubble expansion rate decreases to a value comparable to $\Gamma$ that $\chi$ particle production becomes effective. It is the energy density at the time when $H=\Gamma$ which determines how much energy ends up in $\chi$ particles and thus determines the "reheating temperature", the temperature of the SM fields after energy transfer.

$$
T_{R} \sim\left(\Gamma m_{p l}\right)^{1 / 2}
$$

Since $\Gamma$ is proportional to the square of the coupling constant $g$ which is generally very small, perturbative reheating is slow and produces a reheating temperature which can be very low compared to the energy scale at which inflation takes place.

There are two main problems with the perturbative decay analysis described above. First of all, even if the inflaton decay were perturbative, it is not justified to use the heuristic equation (3.25) since it violates the fluctuation-dissipation theorem: in systems with dissipation, there are always fluctuations, and these are missing in (3.25). For an improved effective equation of motion see e.g. [95].

The main problem with the perturbative analysis is that it does not take into account the coherent nature of the inflaton field $\varphi$. At the beginning of the period of oscillations $\varphi$ is not a superposition of free asymptotic single inflaton states, but rather a coherently oscillating homogeneous field. The large amplitude of oscillation implies that it is well justified to treat the inflaton classically. However, the matter fields can be assumed to start off in their vacuum state (the redshifting during the period of inflation will remove any matter particles present at the beginning of inflation). Thus, matter fields $\chi$ must be treated quantum mechanically. The improved approach to reheating initiated in [91] (see also [96]) is to consider reheating as a quantum production of $\chi$ particles in a classical $\varphi$ background.

We expand the field $\chi$ is terms of the usual creation and annihilation operators. The mode functions satisfy the equation

$$
\ddot{\chi}_{k}+3 H \dot{\chi}+\left(\frac{k^{2}}{a^{2}}+m_{\chi}^{2}+g^{2} \Phi(t)^{2} \sin ^{2}(m t)\right) \chi_{k}=0,
$$

where $\Phi(t)$ is the amplitude of oscillation of $\varphi$.

The first level of approximation [91] is to neglect the expansion of space, to estimate the efficiency of reheating in this approximation, and to check self-consistency of the approximation. The equation (3.27) then reduces to

$$
\ddot{\chi}_{k}+\left(k^{2}+m_{\chi}^{2}+g^{2} \Phi^{2} \sin ^{2}(m t)\right) \chi_{k}=0,
$$


and $\Phi(t)$ is constant. The equation is the Mathieu equation [97] which is conventionally written in the form

$$
\chi_{k}^{\prime \prime}+\left(A_{k}-2 q \cos 2 z\right) \chi_{k}=0,
$$

where we have introduced the dimensionless time variable $z=m t$ and a prime now denotes the derivative with respect to $z$. Comparing the coefficients, we see that

$$
A_{k}=\frac{k^{2}+m_{\chi}^{2}}{m^{2}}+2 q \quad q=\frac{g^{2} \Phi^{2}}{4 m^{2}} .
$$

The equation (3.29) describes parametric resonance in classical mechanics. From the examples in classical mechanics in which this equation arises we know that there are bands of $k$ values (depending on the frequency $m$ of the external source) which exhibit exponential instability. For the general theory of the Mathieu equation the reader is referred to [97]. For values of $k$ in a resonance band, the growth of $\chi_{k}$ can be written as

$$
\chi_{k} \sim e^{\mu_{k} z},
$$

where $\mu_{k}$ is called the Floquet exponent. In the model we are considering in this subsection, resonance occurs for all long wavelength modes - roughly speaking all modes with

$$
k^{2} \ll g m \Phi .
$$

This is called broad-band resonance [92, 94]. The Floquet exponent is of order 1, and this implies very efficient energy transfer from the coherently oscillating inflaton field to a gas of $\chi$ particles. This initial stage of energy transfer is called preheating. The time scale of the energy transfer is short compared to the cosmological time scale $H^{-1}$ at the end of inflation [91]. Hence, the approximation of neglecting the expansion of space is self-consistent.

It is possible to perform an improved analysis which keeps track of the expansion of space. The first step [94] is to rescale the field variable to extract the cosmological red-shifting

$$
X_{k}(t) \equiv a(t) \chi_{k}(t),
$$

and to work in terms of conformal time. The field $X_{k}$ obeys an equation without cosmological damping term which is very similar to (3.29), except that the oscillatory correction term to the mass is replaced by a more general periodic function of the conformal time $\eta$. The Floquet theory of [97] also applies to the resulting equation, and we reach the conclusion that the exponential instability of $\chi$ (modulated by $a(t)$ ) persists when including the effects of the expansion of space. For details the reader is referred to [94] or to the recent review [89].

\subsubsection{Preheating of Metric Perturbations}

A parametric resonance instability should be expected for all fields which couple to the oscillating inflaton condensate. In particular, metric fluctuations should also be effected. As was shown in [54], there is no parametric resonance instability for long wavelength modes in the case of purely adiabatic perturbations (see also [38]). However, in the presence of entropy fluctuations, parametric amplification of cosmological perturbations driven by the oscillating inflaton condensate is possible $[57,58]$ (see also [98]). 
To study the preheating of metric fluctuations, we refer back to the formalism of cosmological perturbations developed in Subsection (2.3.4). In the presence of an entropy perturbation $\delta S$, the "conservation" equation (2.51) for the variable $\zeta$ (which describes the curvature perturbation in comoving gauge) gets replaced by

$$
\dot{\zeta}=\frac{\dot{p}}{p+\rho} \delta S .
$$

If long wavelength fluctuations of a matter field $\chi$ which coupled to the inflaton $\varphi$ ( $\chi$ corresponds to an entropy mode) undergo exponential growth during reheating as they do in the case of a model with broad parametric resonance, then this will induce an exponential growth of $\delta S$ which will via (3.34) induce an exponentially growing curvature perturbation.

For an elegant formalism to compute the source term in (3.34) in terms of the scalar fields $\varphi$ and $\chi$ the reader is referred to [99]. This resonant growth of entropy fluctuations is only important in models in which the entropy fluctuations are not suppressed during inflation. Some recent examples were studied in $[100,101,102]$. The source for this instability need not be the oscillations of the inflaton field. In SUSY models, the decay of flat directions can also induce this instability [103].

\subsection{Problems of Inflation}

In spite of the phenomenological success of the inflationary paradigm, conventional scalar field-driven inflation suffers from several important conceptual problems.

The first problem concern the nature of the inflaton, the scalar field which generates the inflationary expansion. No particle corresponding to the excitation of a scalar field has yet been observed in nature, and the Higgs field which is introduced to give elementary particles masses in the Standard Model of particle physics does not have the required flatness of the potential to yield inflation, unless it is non-minimally coupled to gravity [104]. In particle physics theories beyond the Standard Model there are often many scalar fields, but it is in general very hard to obtain the required flatness properties on the potential

The second problem (the amplitude problem) relates to the amplitude of the spectrum of cosmological perturbations. In a wide class of inflationary models, obtaining the correct amplitude requires the introduction of a hierarchy in scales, namely [105]

$$
\frac{V(\varphi)}{\Delta \varphi^{4}} \leq 10^{-12},
$$

where $\Delta \varphi$ is the change in the inflaton field during the minimal length of the inflationary period, and $V(\varphi)$ is the potential energy during inflation.

A more serious problem is the trans-Planckian problem [106]. Returning to the space-time diagram of inflation (see Figure 10), we can immediately deduce that, provided that the period of inflation lasted sufficiently long (for GUT scale inflation the number is about 70 e-foldings), then all scales inside the Hubble radius today started out with a physical wavelength smaller than the Planck scale at the beginning of inflation. Now, the theory of cosmological perturbations is based on Einstein's theory of General Relativity coupled to a simple semi-classical description of matter. It is clear that these building blocks of the theory are inapplicable on scales comparable and smaller than the Planck scale. Thus, the key successful prediction of inflation (the theory of the 


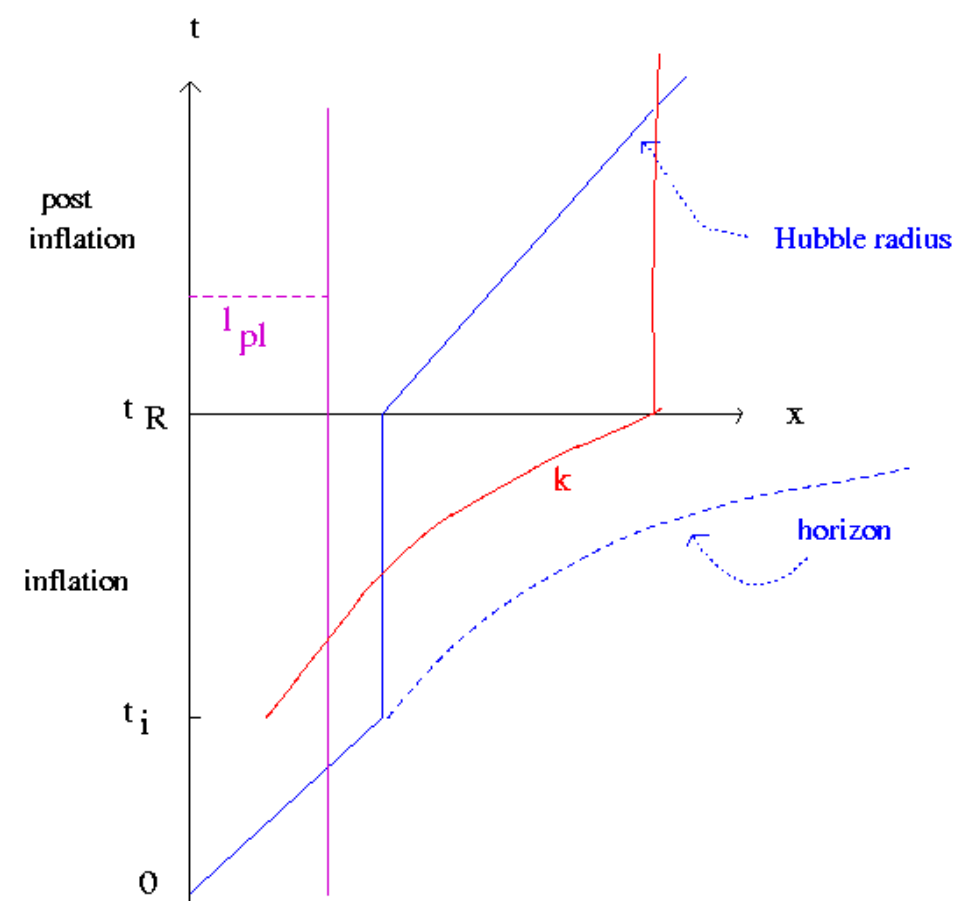

Figure 10: Space-time diagram (sketch) of inflationary cosmology where we have added an extra length scale, namely the Planck length $l_{p l}$ (majenta vertical line). The symbols have the same meaning as in Figure 5. Note, specifically, that - as long as the period of inflation lasts a couple of e-foldings longer than the minimal value required for inflation to address the problems of standard big bang cosmology - all wavelengths of cosmological interest to us today start out at the beginning of the period of inflation with a wavelength which is smaller than the Planck length.

origin of fluctuations) is based on suspect calculations since new physics must enter into a correct computation of the spectrum of cosmological perturbations. The key question is as to whether the predictions obtained using the current theory are sensitive to the specifics of the unknown theory which takes over on small scales.

One approach to study the sensitivity of the usual predictions of inflationary cosmology to the unknown physics on trans-Planckian scales is to study toy models of ultraviolet physics which allow explicit calculations. The first approach which was used $[107,108]$ is to replace the usual linear dispersion relation for the Fourier modes of the fluctuations by a modified dispersion relation, a dispersion relation which is linear for physical wavenumbers smaller than the scale of new physics, but deviates on larger scales. Such dispersion relations were used previously to test the sensitivity of black hole radiation on the unknown physics of the UV [109, 110]. It was found [107] that if the evolution of modes on the trans-Planckian scales is non-adiabatic, then substantial deviations of the spectrum of fluctuations from the usual results are possible. Non-adiabatic evolution turns an initial state minimizing the energy density into a state which is excited once the wavelength becomes larger than the cutoff scale. Back-reaction effects of these excitations may limit the magnitude of the trans-Planckian effects if we assume that inflation took place $[111,112,113]$. On the other hand, large trans-Planckian effects may also prevent the onset of inflation.

A fourth problem is the singularity problem. It was known for a long time that standard Big 
Bang cosmology cannot be the complete story of the early universe because of the initial singularity, a singularity which is unavoidable when basing cosmology on Einstein's field equations in the presence of a matter source obeying the weak energy conditions (see e.g. [114] for a textbook discussion). Recently, the singularity theorems have been generalized to apply to Einstein gravity coupled to scalar field matter, i.e. to scalar field-driven inflationary cosmology [115]. It was shown that, in this context, a past singularity at some point in space is unavoidable. Thus we know, from the outset, that scalar field-driven inflation cannot be the ultimate theory of the very early universe.

The Achilles heel of scalar field-driven inflationary cosmology may be the cosmological constant problem. We know from observations that the large quantum vacuum energy of field theories does not gravitate today. However, to obtain a period of inflation one is using the part of the energy-momentum tensor of the scalar field which looks like the vacuum energy. In the absence of a solution of the cosmological constant problem it is unclear whether scalar field-driven inflation is robust, i.e. whether the mechanism which renders the quantum vacuum energy gravitationally inert today will not also prevent the vacuum energy from gravitating during the period of slow-rolling of the inflaton field. Note that the approach to addressing the cosmological constant problem making use of the gravitational back-reaction of long range fluctuations (see [116] for a summary of this approach) does not prevent a long period of inflation in the early universe.

A final problem which we will mention here is the concern that the energy scale at which inflation takes place is too high to justify an effective field theory analysis based on Einstein gravity. In simple toy models of inflation, the energy scale during the period of inflation is about $10^{16} \mathrm{GeV}$, very close to the string scale in many string models, and not too far from the Planck scale. Thus, correction terms in the effective action for matter and gravity may already be important at the energy scale of inflation, and the cosmological dynamics may be rather different from what is obtained when neglecting the correction terms.

In Figure 11 we show once again the space-time sketch of inflationary cosmology. In addition to the length scales which appear in the previous versions of this figure, we have now shaded the "zones of ignorance", zones where the Einstein gravity effective action is sure to break down. As described above, fluctuations emerge from the short distance zone of ignorance (except if the period of inflation is very short), and the energy scale of inflation might put the period of inflation too close to the high energy density zone of ignorance to trust the predictions based on using the Einstein action.

The arguments in this subsection provide a motivation for considering alternative scenarios of early universe cosmology. Below we will focus on two scenarios, the matter bounce and string gas cosmology. It is important to emphasize that these are not the only alternative scenarios. Others include the Pre-Big-Bang scenario [117] and the Ekpyrotic paradigm [118].

\section{Matter Bounce}

The matter bounce scenario is a non-singular cosmology in which time runs from $-\infty$ to $+\infty$. Negative times correspond to a contracting phase, positive times to expansion. It is assumed that the bounce phase is short ${ }^{9}$.

\footnotetext{
${ }^{9}$ Short means short compared to the time it takes light to travel over distances of about $1 \mathrm{~mm}$, the physical length
} 


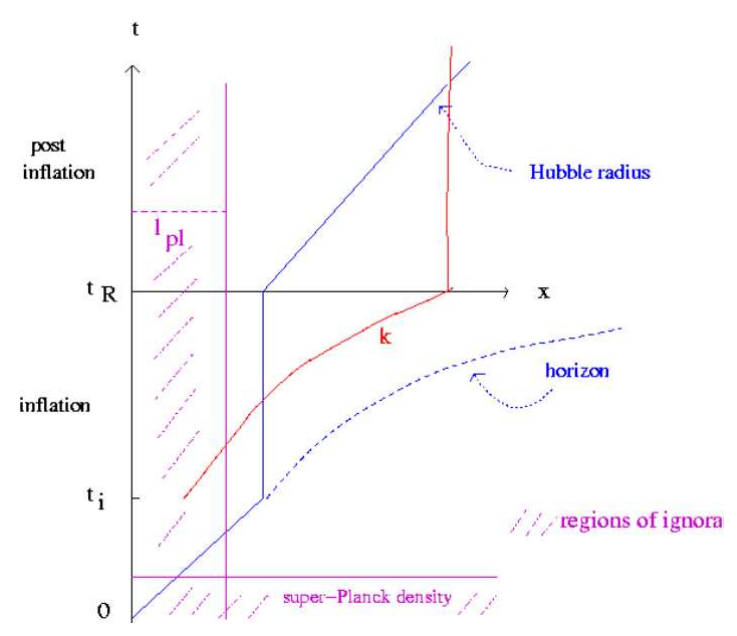

Figure 11: Space-time diagram (sketch) of inflationary cosmology including the two zones of ignorance sub-Planckian wavelengths and trans-Planckian densities. The symbols have the same meaning as in Figure 5. Note, specifically, that - as long as the period of inflation lasts a couple of e-foldings longer than the minimal value required for inflation to address the problems of standard big bang cosmology - all wavelengths of cosmological interest to us today start out at the beginning of the period of inflation with a wavelength which is in the zone of ignorance.

As indicated in Figure 6, the fluctuations which we observe today have exited the Hubble radius early in the contracting phase. The word matter in matter bounce should emphasize that we are assuming that the phase during which the fluctuations which we observe today are exiting the Hubble radius is dominated by cold pressure-less matter. Viewing the contracting phase as the time reverse of the expanding phase we are living in, this last assumption we have made does not seem very restrictive. In fact, one could expect that some entropy is generated during the bounce, in which case the matter phase during the contracting period would last up to higher energy densities than it does in the expanding phase.

\subsection{Models for a Matter Bounce and Background Cosmology}

It follows from the singularity theorems of General Relativity (see e.g. [114]) that in order to obtain a non-singular bounce we must either invoke matter which violates the weak energy condition, or go beyond General Relativity. A review of ways to obtain a non-singular cosmology is given in [20]. The major challenges in constructing a non-singular bouncing cosmology include

which corresponds to to the largest comoving scales we observe today (assuming that the energy scale of the bounce is about $\left.10^{16} \mathrm{GeV}\right)$. 
the following: first, the model should be free of ghost-like excitations, since a model with ghosts will be unstable [119]. Next, the background cosmology should be stable towards the addition of radiation to the matter sector. Finally, the homogeneous and isotropic background trajectory should also be stable against anisotropic stress.

In the following we will only mention a few recent attempts which the author of these lecture notes has been involved in. We will first discuss models obtained by modifying the matter sector of the theory, and then models where the gravitational sector of the theory is changed.

Introducing quintom matter [120] yields a way of obtaining a non-singular bouncing cosmology, as discussed in [19]. Quintom matter is a set of two matter fields, one of them regular matter (obeying the weak energy condition), the second a field with opposite sign kinetic term, a field which violates the energy conditions. We can [19] model both matter components with scalar fields. Let us denote the mass of the regular one $(\varphi)$ by $m$, and by $M$ that of the field $\tilde{\varphi}$ with wrong sign kinetic term. We assume that early in the contracting phase both fields are oscillating, but that the amplitude $\mathscr{A}$ of $\varphi$ greatly exceeds the corresponding amplitude $\tilde{\mathscr{A}}$ of $\tilde{\varphi}$ such that the energy density is dominated by $\varphi$. Both fields will initially be oscillating during the contracting phase, and both amplitudes grow at the same rate. At some point, $\mathscr{A}$ will become so large that the oscillations of $\varphi$ freeze out ${ }^{10}$. Then, $\mathscr{A}$ will grow only slowly, whereas $\tilde{\mathscr{A}}$ will continue to increase. Thus, the (negative) energy density in $\tilde{\varphi}$ will grow in absolute values relative to that of $\varphi$. The total energy density will decrease towards 0 . At that point, $H=0$ by the Friedmann equations. It can in fact easily be seen that $\dot{H}>0$ when $H=0$. Hence, a non-singular bounce occurs. The Higgs sector of the Lee-Wick model [121] provides a concrete realization of the quintom bounce model, as studied in [25]. Quintom models like all other models with negative sign kinetic terms suffer from an instability problem [119] in the matter sector and are hence problematic. In addition, they are unstable against the addition of radiation (see e.g. [122]) and anisotropic stress.

An improved way of obtaining a non-singular bouncing cosmology using modified matter is by using a ghost condensate field [123]. The ghost condensation mechanism is the analog of the Higgs mechanism in the kinetic sector of the theory. In the Higgs mechanism we take a field $\phi$ whose mass when evaluated at $\phi=0$ is tachyonic, add higher powers of $\phi^{2}$ to the potential term in the Lagrangian such that there is a stable fixed point $\phi=v \neq 0$, and thus when expanded about $\phi=v$ the mass term has the "safe" non-tachyonic sign. In the ghost condensate construction we take a field $\phi$ whose kinetic term

$$
X \equiv-g^{\mu v} \partial_{\mu} \phi \partial_{v} \phi
$$

appears with the wrong sign in the Lagrangian. Then, we add higher powers of $X$ to the kinetic Lagrangian such that there is a stable fixed point $X=c^{2}$ and such that when expanded about $X=c^{2}$ the fluctuations have the regular sign of the kinetic term:

$$
\mathscr{L}=\frac{1}{8} M^{4}\left(X-c^{2}\right)^{2}-V(\phi),
$$

where $V(\phi)$ is a usual potential function, $M$ is a characteristic mass scale and the dimensions of $\phi$ are chosen such that $X$ is dimensionless.

In the context of cosmology, the ghost condensate is

$$
\phi=c t
$$

\footnotetext{
${ }^{10}$ This corresponds to the time reverse of entering a region of large-field inflation.
} 
and breaks local Lorentz invariance. Now let us expand the homogeneous component of $\phi$ about the ghost condensate:

$$
\phi(t)=c t+\pi(t) .
$$

If $\dot{\pi}<0$ then the gravitational energy density is negative, and a non-singular bounce is possible. Thus, in [123] we constructed a model in which the ghost condensate field starts at negative values and the potential $V(\phi)$ is negligible. As $\phi$ approaches $\phi=0$ it encounters a positive potential which slows it down, leading to $\pi<0$ and hence to negative gravitational energy density. Thus, a non-singular bounce can occur. We take the potential to be of the form

$$
V(\phi) \sim \phi^{-\alpha}
$$

for $|\phi| \gg M$, where $M$ is the mass scale above which the higher derivative kinetic terms are important. For sufficiently large values of $\alpha$, namely

$$
\alpha \geq 6
$$

the energy density in the ghost condensate increases faster than that of radiation and anisotropic stress at the universe contracts. Hence, this bouncing cosmology is stable against the addition of radiation and anisotropic stress.

Turning to the second way of obtaining a non-singular bounce, namely by modifying the gravitational action, we should emphasize that modifications of the gravitational action are expected at the high densities at which the bounce will occur. Concrete examples were already mentioned in the Introduction: the higher derivative Lagrangian resulting from the nonsingular universe construction of [17], the model of [16] which is based on a non-local higher derivative action which is ghostfree about Minkowski space-time, mirage cosmologies [125] resulting from the effective action of gravity on a brane which is moving into and out of a high-curvature throat in a higher-dimensional space-time.

More recently, interest has focused on a non-singular bouncing cosmology which emerges from Horava-Lifshitz gravity [22]. Horava-Lifshitz gravity is a power-counting renormalizable quantum theory of gravity which is based on anisotropic scaling between space and time. In one sense it is a very conservative approach to quantum gravity in the sense that the dynamical degrees of freedom are the usual ones: the spatial metric $g_{i j}$, the lapse function $N$ and the shift vector $N_{i}$. There are no extra dimensions or new degrees of freedom such as strings. On the other hand, it is a very radical approach in the sense that it gives up local Lorentz invariance and space-time diffeomorphism invariance. The residual symmetries are local rotational invariance and spatial diffeomorphism. On the other hand, there is a scaling symmetry under

$$
x \rightarrow l x, t \rightarrow l^{3} t
$$

where $l$ is the scaling parameter. The Lagrangian is constructed to contain all terms which are consistent with the residual symmetries and with power-counting renormalizability with respect to the above scaling symmetry. The action does not contain any higher time derivative terms, but it does contain higher space derivative terms. These terms dominate in the ultraviolet. In the infrared, the extra terms are sub-dominant. Thus, in the infrared the theory is supposed to flow towards General Relativity. 
The extra spatial derivative terms in the gravitational action have an important effect in the very early universe at high energy densities. As shown in [21], if the spatial sections have a nonvanishing spatial curvature constant $k$, then the higher-derivative terms in the action will lead to terms in the Friedmann equations which act as ghost radiation and ghost anisotropic stress, i.e. terms of gravitational origin and negative effective energy density which scale as $a^{-4}$ and $a^{-6}$, respectively. Starting with a contracting universe dominated by regular matter, eventually the ghost terms will catch up and yield a non-singular bounce in analogy to how the ghost matter in the quintom model does.

The analysis of the spectrum of cosmological perturbations on scales of current observational interest is, however, independent of the details of the bouncing phase, as long as that phase is short compared to the time it takes light to travel over the length scales of current interest, and as long as no extra degrees of freedom (extra when compared to those arising in Einstein gravity) become important ${ }^{11}$. We now turn to a discussion of the evolution of fluctuations in a generic matter bounce model.

\subsection{Fluctuations in a Matter Bounce}

First we will consider fluctuations in a matter bounce without extra degrees of freedom. In this case, we need only focus on the usual fluctuation variable $v$. The equation of motion its Fourier mode $v_{k}$ is

$$
v_{k}^{\prime \prime}+\left(k^{2}-\frac{z^{\prime \prime}}{z}\right) v_{k}=0
$$

If the equation of state of the background is time-independent, then $z \sim a$ and hence the negative square mass term in (4.8) is $H^{2}$. Thus, on length scales smaller than the Hubble radius, the solutions of (4.8) are oscillating, whereas on larger scales they are frozen in, and their amplitude depends on the time evolution of $z$.

In the case of an expanding universe the dominant mode of $v$ scales as $z$. However, in a contracting universe it is the second of the two modes which dominates. If the contracting phase is matter-dominated, i.e. $a(t) \sim t^{2 / 3}$ and $\eta(t) \sim t^{1 / 3}$ the dominant mode of $v$ scales as $\eta^{-1}$ and hence

$$
v_{k}(\eta)=c_{1} \eta^{2}+c_{2} \eta^{-1},
$$

where $c_{1}$ and $c_{2}$ are constants. The $c_{1}$ mode is the mode for which $\zeta$ is constant on super-Hubble scales. However, in a contracting universe it is the $c_{2}$ mode which dominates and leads to a scaleinvariant spectrum $[13,14,15]$ :

$$
\begin{aligned}
P_{\zeta}(k, \eta) & \sim k^{3}\left|v_{k}(\eta)\right|^{2} a^{-2}(\eta) \\
& \sim k^{3}\left|v_{k}\left(\eta_{H}(k)\right)\right|^{2}\left(\frac{\eta_{H}(k)}{\eta}\right)^{2} \sim k^{3-1-2} \\
& \sim \text { const },
\end{aligned}
$$

where we have made use of the scaling of the dominant mode of $v_{k}$, the Hubble radius crossing condition $\eta_{H}(k) \sim k^{-1}$, and the assumption that we have a vacuum spectrum at Hubble radius crossing.

\footnotetext{
${ }^{11}$ This last condition is very relevant in the case of the Horava-Lifshitz bounce where we do have to worry about extra gravitational degrees of freedom. We will come back to this issue at the end of the following subsection.
} 
Up to this point, the analysis shows that in the contracting phase the curvature fluctuations are scale-invariant. In non-singular bouncing cosmologies, the fluctuations can be followed in an unambiguous way through the bounce. This was done in the case of the higher derivative bounce model of [16] in [23], and in a non-singular bouncing mirage cosmology model the analysis was performed in [18]. In the case of the Quintom and Lee-Wick bounces, respectively, the fluctuations were followed through the bounce in [24] and [25], respectively. Finally, in the case of the nonprojectable version ${ }^{12}$ of the HL bounce, the evolution of the fluctuations through the bounce was recently studied in [26].

The equations of motion can be solved numerically without approximation. Alternatively, we can solve them approximately using analytical techniques. Key to the analytical analysis are the General Relativistic matching conditions for fluctuations across a phase transition in the background $[126,127]$. These conditions imply that both $\Phi$ and $\tilde{\zeta}$ are conserved at the bounce, where

$$
\tilde{\zeta}=\zeta+\frac{1}{3} \frac{k^{2} \Phi}{\mathscr{H}^{2}-\mathscr{H}^{\prime}}
$$

However, as stressed in [128], these matching conditions can only be used at a transition when the background metric obeys the matching conditions. This is not the case if we were to match directly between the contracting matter phase and the expanding matter phase.

In the case of a non-singular bounce we have three phases: the initial contracting phase with a fixed equation of state (e.g. $w=0$ ), a bounce phase during which the universe smoothly transits between contraction and expansion, and finally the expanding phase with constant $w$. We need to apply the matching conditions twice: first at the transition between the contracting phase and the bounce phase (on both sides of the matching surface the universe is contracting), and then between the bouncing phase and the expanding phase. The bottom line of the studies of [23, 18, $24,25,26]$ is that on length scales large compared to the time of the bounce, the spectrum of curvature fluctuations is not changed during the bounce phase. Since typically the bounce time is set by a microphysical scale whereas the wavelength of fluctuations which we observe today is macroscopic (about 1mm if the bounce scale is set by the particle physics GUT scale), we conclude that for scales relevant to current observations the spectrum is unchanged during the bounce. This completes the demonstration that a non-singular matter bounce leads to a scale-invariant spectrum of cosmological perturbations after the bounce provided that the initial spectrum on sub-Hubble scales is vacuum. Initial thermal fluctuations were followed through the bounce in [27]. Note that perturbations are processed during a bounce and this implies that even if the background cosmology were cyclic, the full evolution including linear fluctuations would be non-cyclic [32].

The above analysis is applicable only as long as no new degrees of freedom become relevant at high energy densities, in particular during the bounce phase. In non-singular bounce models obtained by modifying the matter sector, new degrees of freedom arise from the extra matter fields. They can thus give entropy fluctuations which may compete with the adiabatic mode studied above. In the quintom bounce model this issue has recently been studied in [129]. It was found that fluctuations in the ghost field which yields the bounce are unimportant on large scales since they have a blue spectrum. However, entropy fluctuations due to extra low-mass fields can be important. They yield the "matter bounce curvaton" mechanism. Their spectrum is also scale-invariant.

\footnotetext{
${ }^{12}$ See below for the specification of this case.
} 
In non-singular bouncing models obtained by modifying the gravitational sector of the theory the identification of potential extra degrees of freedom is more difficult. As an example, let us mention the situation in the case of the Horava-Lifshitz bounce. The theory has the same number of geometric degrees of freedom as General Relativity, but less symmetries. Thus, more of the degrees of freedom are physical. Recall from the discussion of the theory of cosmological perturbations in Section 2 that there are ten total geometrical degrees of freedom for linear cosmological perturbations, four of them being scalar, four vector and two tensor. In Einstein gravity the symmetry group of space-time diffeomorphisms is generated at the level of linear fluctuations by four functions, leaving six of the ten geometrical variables as physical - two scalar, two vector and two tensor modes. In the absence of anisotropic stress the number of scalar variables is reduced by one, and the Hamiltonian constraint relates the remaining scalar metric fluctuation to matter.

In Horava-Lifshitz gravity one loses one scalar gauge degree of freedom, namely that of spacedependent time reparametrizations. Thus, one expects an extra physical degree of freedom. It has been recently been shown [130] that in the projectable version of the theory (in which the lapse function $N(t)$ is constrainted to be a function of time only) the extra degree of scalar cosmological perturbations is either ghost-like or tachyonic, depending on parameters in the Lagrangian. Thus, the theory appears to be ill-behaved in the context of cosmology. However, in the full nonprojectable version (in which the lapse $N(t, \mathbf{x})$ is a function of both space and time, the extra degree of freedom is well behaved. It is important on ultraviolet scales but decouples in the infrared [131].

\subsection{Key Predictions of the Matter Bounce}

Canonical single field inflation models predict very small non-Gaussianities in the spectrum of fluctuations. One way to characterize the non-Gaussianities is via the three point function of the curvature fluctuation, also called the "bispectrum". As realized in [132], the bispectrum induced in the minimal matter bounce scenario (no entropy modes considered) has an amplitude which is at the borderline of what the Planck satellite experiment will be able to detect, and it has a special form. These are specific predictions of the matter bounce scenario with which the matter bounce scenario can be distinguished from those of standard inflationary models (see [133] for a recent detailed review of non-Gaussianities in inflationary cosmology and a list of references). In the following we give a very brief summary of the analysis of non-Gaussianities in the matter bounce scenario.

Non-Gaussianities are induced in any cosmological model simply because the Einstein equations are non-linear. In momentum space, the bispectrum contains amplitude and shape information. The bispectrum is a function of the three momenta. Momentum conservation implies that the three momenta have to add up to zero. However, this still leaves a rich shape information in the bispectrum in addition to the information about the overall amplitude.

A formalism to compute the non-Gaussianities for the curvature fluctuation variable $\zeta$ was developed in [134]. Working in the interaction representation, the three-point function of $\zeta$ is given to leading order by

$$
\begin{aligned}
& <\zeta\left(t, \vec{k}_{1}\right) \zeta\left(t, \vec{k}_{2}\right) \zeta\left(t, \vec{k}_{3}\right)> \\
& =i \int_{t_{i}}^{t} d t^{\prime}<\left[\zeta\left(t, \vec{k}_{1}\right) \zeta\left(t, \vec{k}_{2}\right) \zeta\left(t, \vec{k}_{3}\right), L_{i n t}\left(t^{\prime}\right)\right]>,
\end{aligned}
$$


where $t_{i}$ corresponds to the initial time before which there are any non-Gaussianities. The square parentheses indicate the commutator, and $L_{\text {int }}$ is the interaction Lagrangian

The interaction Lagrangian contains many terms. In particular, there are terms containing the time derivative of $\zeta$. Each term leads to a particular shape of the bispectrum. In an expanding universe such as in inflationary cosmology $\dot{\zeta}=0$. However, in a contracting phase the time derivative of $\zeta$ does not vanish since the dominant mode is growing in time. Hence, there are new contributions to the shape which have a very different form from the shape of the terms which appear in inflationary cosmology. The larger value of the amplitude of the bispectrum follows again from the fact that there is a mode function which grows in time in the contracting phase.

The three-point function can be expressed in the following general form:

$$
\begin{aligned}
<\zeta\left(\vec{k}_{1}\right) \zeta\left(\vec{k}_{2}\right) \zeta\left(\vec{k}_{3}\right)>= & (2 \pi)^{7} \delta\left(\sum \vec{k}_{i}\right) \frac{P_{\zeta}^{2}}{\prod k_{i}^{3}} \\
& \times \mathscr{A}\left(\vec{k}_{1}, \vec{k}_{2}, \vec{k}_{3}\right),
\end{aligned}
$$

where $k_{i}=\left|\vec{k}_{i}\right|$ and $\mathscr{A}$ is the shape function. In this expression we have factored out the dependence on the power spectrum $P_{\zeta}$. In inflationary cosmology it has become usual to express the bispectrum in terms of a non-Gaussianity parameter $f_{N L}$. However, this is only useful if the shape of the three point function is known. As a generalization, we here use [132]

$$
|\mathscr{B}|_{N L}\left(\vec{k}_{1}, \vec{k}_{2}, \vec{k}_{3}\right)=\frac{10}{3} \frac{\mathscr{A}\left(\vec{k}_{1}, \vec{k}_{2}, \vec{k}_{3}\right)}{\sum_{i} k_{i}^{3}}
$$

The computation of the bispectrum is tedious. In the case of the matter bounce (no entropy fluctuations) the result is

$$
\begin{aligned}
\mathscr{A}= & \frac{3}{256 \prod k_{i}^{2}}\left\{3 \sum k_{i}^{9}+\sum_{i \neq j} k_{i}^{7} k_{j}^{2}\right. \\
& -9 \sum_{i \neq j} k_{i}^{6} k_{j}^{3}+5 \sum_{i \neq j} k_{i}^{5} k_{j}^{4} \\
& \left.-66 \sum_{i \neq j \neq k} k_{i}^{5} k_{j}^{2} k_{k}^{2}+9 \sum_{i \neq j \neq k} k_{i}^{4} k_{j}^{3} k_{k}^{2}\right\} .
\end{aligned}
$$

This equation describes the shape which is predicted. Some of the terms (such as the last two) are the same as those which occur in single field slow-roll inflation, but the others are new. Note, in particular, that the new terms are not negligible.

If we project the resulting shape function $\mathscr{A}$ onto some popular shape masks we

$$
|\mathscr{B}|_{N L}^{\text {local }}=-\frac{35}{8},
$$

for the local shape $\left(k_{1} \ll k_{2}=k_{3}\right)$. This is negative and of order $O(1)$. For the equilateral form $\left(k_{1}=k_{2}=k_{3}\right)$ the result is

$$
|\mathscr{B}|_{N L}^{\text {equil }}=-\frac{255}{64}
$$


and for the folded form $\left(k_{1}=2 k_{2}=2 k_{3}\right)$ one obtains the value

$$
|\mathscr{B}|_{N L}^{\text {folded }}=-\frac{9}{4} .
$$

These amplitudes are close to what the Planck CMB satellite experiment will be able to detect.

The matter bounce scenario also predicts a change in the slope of the primordial power spectrum on small scales [135]: scales which exit the Hubble radius in the radiation phase retain a blue spectrum since the squeezing rate on scales larger than the Hubble radius is insufficient to give longer wavelength modes a sufficient boost relative to the shorter wavelength ones.

\subsection{Problems of the Matter Bounce}

The main problem of the matter bounce scenario is that the physics which yields the nonsingular bounce is not (yet) well established. It is clearly required to go beyond conventional quantum field theory coupled to General Relativity to obtain such a bounce. New Planck-scale physics needs to be invoked to obtain the required background cosmology. We remind the reader that it is precisely the absence of such new physics which needs to be invoked to argue for an inflationary background evolution.

In terms of the trans-Planckian problem for cosmological fluctuations, the bouncing cosmology has a clear advantage: the wavelength of the fluctuations which we are interested in always remains in the far infrared compared to the ultraviolet scales of the new physics. It can be shown that the correction terms due to the new ultraviolet physics on the evolution of fluctuations of interest to observers are negligible (see e.g. [26]).

The second main problem of the matter bounce scenario may be the sensitivity of the bounce to assumptions on the initial conditions in the far past. The amplitude of the classical initial fluctuations must be small in order for the homogeneous background dynamics not to be perturbed, and for the vacuum fluctuations to dominate on the scales relevant in the infrared (see e.g. [136] for a criticism of initial conditions in a related scenario, the Pre-Big-Bang scenario). In addition, the initial shear must be very small. In some bouncing cosmology backgrounds the bouncing solution is unstable to the smallest addition of shear. This, however, is not the case in other models such as the Horava-Lifshitz bounce [21] or the ghost condensate bounce [123].

The matter bounce scenario does not address the cosmological constant problem, but on the other hand the scenario does not depend on how the cosmological constant problem is solved. Note that inflationary cosmology is NOT robust in this respect.

\section{String Gas Cosmology}

Another scenario of early universe cosmology which can provide an explanation for the current data is string gas cosmology. It is an "emergent universe" [137] scenario in which the universe begins in a long hot and almost static phase. The key input from string theory is that matter is a gas of closed fundamental strings compared to a gas of point particles as is assumed in Standard Big Bang cosmology. 


\subsection{Principles and Background}

\subsubsection{Principles}

String theory may be the best candidate we have at the present time for a quantum theory of gravity which unifies all four forces of nature. This theory is, however, currently not yet well understood beyond perturbation theory ${ }^{13}$. For applications to early universe cosmology, however, a non-perturbative understanding will be essential.

In the absence of a non-perturbative formulation of string theory, the approach to string cosmology which we have suggested, string gas cosmology [28] (see also [29], and [11, 139, 140] for reviews and more complete list of references), is to focus on symmetries and degrees of freedom which are new to string theory (compared to point particle theories) and which will be part of any non-perturbative string theory, and to use them to develop a new cosmology. The symmetry we make use of is T-duality, and the new degrees of freedom are the string oscillatory modes and the string winding modes.

String gas cosmology is based on coupling a classical background which includes the graviton and the dilaton fields to a gas of closed strings (and possibly other basic degrees of freedom of string theory such as "branes" [141]). All dimensions of space are taken to be compact, for reasons which will become clear later. For simplicity, we take all spatial directions to be toroidal and denote the radius of the torus by $R$. Strings have three types of states: momentum modes which represent the center of mass motion of the string, oscillatory modes which represent the fluctuations of the strings, and winding modes counting the number of times a string wraps the torus.

Since the number of string oscillatory states increases exponentially with energy, there is a limiting temperature for a gas of strings in thermal equilibrium, the Hagedorn temperature [30] $T_{H}$. Thus, if we take a box of strings and adiabatically decrease the box size, the temperature will never diverge. This is the first indication that string theory has the potential to resolve the cosmological singularity problem.

The second key feature of string theory upon which string gas cosmology is based is T-duality. To introduce this symmetry, let us discuss the radius dependence of the energy of the basic string states: The energy of an oscillatory mode is independent of $R$, momentum mode energies are quantized in units of $1 / R$, i.e.

$$
E_{n}=n \mu \frac{l_{s}^{2}}{R}
$$

where $l_{s}$ is the string length and $\mu$ is the mass per unit length of a string. The winding mode energies are quantized in units of $R$, i.e.

$$
E_{m}=m \mu R
$$

where both $n$ and $m$ are integers. Thus, a new symmetry of the spectrum of string states emerges: Under the change

$$
R \rightarrow 1 / R
$$

\footnotetext{
${ }^{13}$ Note that there are concrete proposals for a non-perturbative formulation such as the AdS/CFT correspondence [138].
} 
in the radius of the torus (in units of $l_{s}$ ) the energy spectrum of string states is invariant if winding and momentum quantum numbers are interchanged

$$
(n, m) \rightarrow(m, n) .
$$

The above symmetry is the simplest element of a larger symmetry group, the T-duality symmetry group which in general also mixes fluxes and geometry. The string vertex operators are consistent with this symmetry, and thus T-duality is a symmetry of perturbative string theory. Postulating that T-duality extends to non-perturbative string theory leads [142] to the need of adding D-branes to the list of fundamental objects in string theory. With this addition, T-duality is expected to be a symmetry of non-perturbative string theory. Specifically, T-duality will take a spectrum of stable Type IIA branes and map it into a corresponding spectrum of stable Type IIB branes with identical masses [143].

As discussed in [28], the above T-duality symmetry leads to an equivalence between small and large spaces, an equivalence elaborated on further in [144, 145].

\subsubsection{Background Cosmology}

That string gas cosmology will lead to a dynamical evolution of the early universe very different from what is obtained in standard and inflationary cosmology can already be seen by combining the basic ingredients from string theory discussed in the previous subsection. As the radius of a box of strings decreases from an initially very large value - maintaining thermal equilibrium - , the temperature first rises as in standard cosmology since the string states which are occupied (the momentum modes) get heavier. However, as the temperature approaches the Hagedorn temperature, the energy begins to flow into the oscillatory modes and the increase in temperature levels off. As the radius $R$ decreases below the string scale, the temperature begins to decrease as the energy begins to flow into the winding modes whose energy decreases as $R$ decreases (see Figure 12). Thus, as argued in [28], the temperature singularity of early universe cosmology should be resolved in string gas cosmology.

The equations that govern the background of string gas cosmology are not known. The Einstein equations are not the correct equations since they do not obey the T-duality symmetry of string theory. Many early studies of string gas cosmology were based on using the dilaton gravity equations [146, 147, 148]. However, these equations are not satisfactory, either. Firstly, we expect that string theory correction terms to the low energy effective action of string theory become dominant in the Hagedorn phase. Secondly, the dilaton gravity equations yield a rapidly changing dilaton during the Hagedorn phase (in the string frame). Once the dilaton becomes large, it becomes inconsistent to focus on fundamental string states rather than brane states. In other words, using dilaton gravity as a background for string gas cosmology does not correctly reflect the S-duality symmetry of string theory. Recently, a background for string gas cosmology including a rolling tachyon was proposed [149] which allows a background in the Hagedorn phase with constant scale factor and constant dilaton. Another study of this problem was given in [150].

Some conclusions about the time-temperature relation in string gas cosmology can be derived based on thermodynamical considerations alone. One possibility is that $R$ starts out much smaller than the self-dual value and increases monotonically. From Figure 12 it then follows that the timetemperature curve will correspond to that of a bouncing cosmology. Alternatively, it is possible 


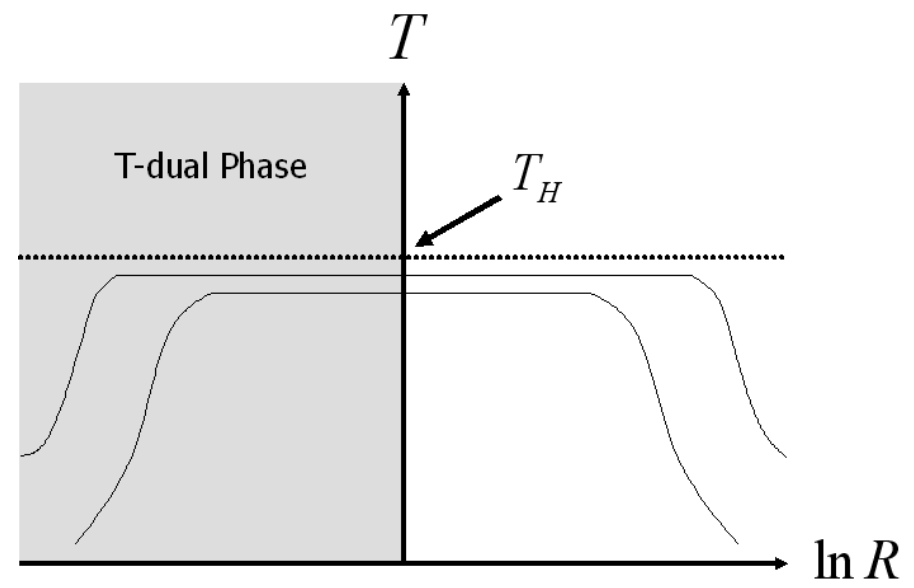

Figure 12: The temperature (vertical axis) as a function of radius (horizontal axis) of a gas of closed strings in thermal equilibrium. Note the absence of a temperature singularity. The range of values of $R$ for which the temperature is close to the Hagedorn temperature $T_{H}$ depends on the total entropy of the universe. The upper of the two curves corresponds to a universe with larger entropy.

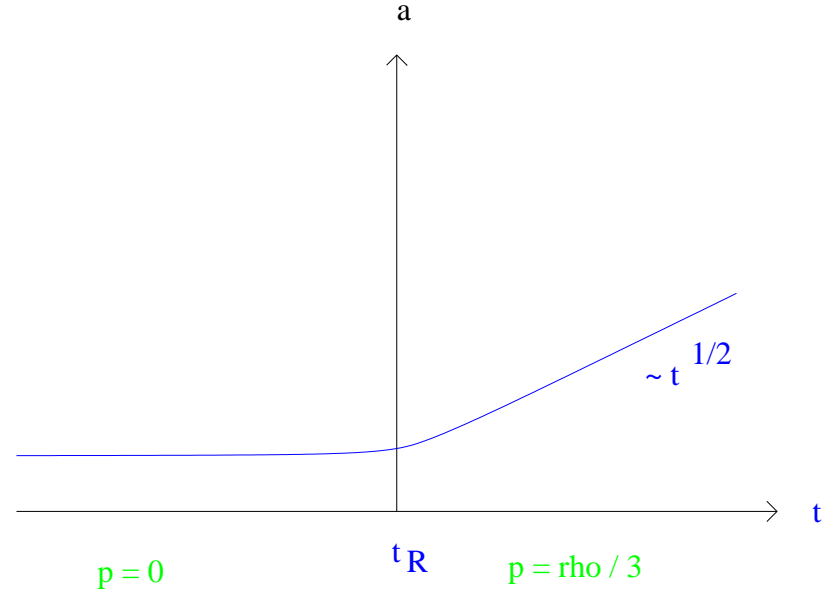

Figure 13: The dynamics of string gas cosmology. The vertical axis represents the scale factor of the universe, the horizontal axis is time. Along the horizontal axis, the approximate equation of state is also indicated. During the Hagedorn phase the pressure is negligible due to the cancellation between the positive pressure of the momentum modes and the negative pressure of the winding modes, after time $t_{R}$ the equation of state is that of a radiation-dominated universe.

that the universe starts out in a meta-stable state near the Hagedorn temperature, the Hagedorn phase, and then smoothly evolves into an expanding phase dominated by radiation like in standard cosmology (Figure 13). Note that we are assuming that not only the scale factor but also the dilaton is constant in time.

The transition between the quasi-static Hagedorn phase and the radiation phase at the time $t_{R}$ is a consequence of the annihilation of string winding modes into string loops (see Figure 14). Since this process corresponds to the production of radiation, we denote this time by the same symbol $t_{R}$ as the time of reheating in inflationary cosmology. As pointed out in [28], this annihilation 


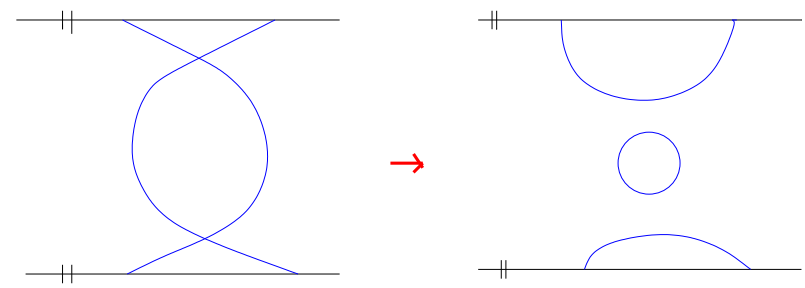

Figure 14: The process by which string loops are produced via the intersection of winding strings. The top and bottom lines are identified and the space between these lines represents space with one toroidal dimension un-wrapped.

process only is possible in at most three large spatial dimensions. This is a simple dimension counting argument: string world sheets have measure zero intersection probability in more than four large space-time dimensions. Hence, string gas cosmology may provide a natural mechanism for explaining why there are exactly three large spatial dimensions. This argument was supported by numerical studies of string evolution in three and four spatial dimensions [151] (see also [152]). The flow of energy from winding modes to string loops can be modelled by effective Boltzmann equations [153] analogous to those used to describe the flow of energy between infinite cosmic strings and cosmic string loops (see e.g. [86, 87, 88] for reviews).

Several comments are in place concerning the above mechanism. First, in the analysis of [153] it was assumed that the string interaction rates were time-independent. If the dynamics of the Hagedorn phase is modelled by dilaton gravity, the dilaton is rapidly changing during the phase in which the string frame scale factor is static. As discussed in [154, 155] (see also [156]), in this case the mechanism which tells us that exactly three spatial dimensions become macroscopic does not work. Another comment concerns the isotropy of the three large dimensions. In contrast to the situation in Standard cosmology, in string gas cosmology the anisotropy decreases in the expanding phase [157]. Thus, there is a natural isotropization mechanism for the three large spatial dimensions.

\subsubsection{Moduli Stabilization}

In the following, we shall assume that either the mechanism of [28] for setting in motion the preferential expansion of exactly three spatial dimensions works, or, alternatively, that three dimensions are distinguished from the beginning as being large. In either case, we must address the moduli stabilization problem, i.e. we must show that the radii (radions) and shapes of the extra dimensions are stabilized. This is a major challenge in string-motivated field theory models of higher dimensions. The situation in string gas cosmology is much better in comparison: all moduli except for the dilaton are stabilized without the need of introducing extra ingredients such as fluxes or special non-perturbative effects.

Radion stabilization in the string frame was initially studied in [158]. The basic idea is that the winding modes about the extra spatial dimensions provide a confining force which prevents the radii from increasing whereas the momentum modes provide a force which resists the complete contraction. Thus, there will be a stable minimum of the effective potential for the radion.

In order to make contact with late time cosmology, it is important to consider the issue of radion stabilization when the dilaton is frozen, or, more generally, in the Einstein frame. As was 
discussed in $[159,160]$ (see also earlier comments in [158]), the existence of string modes which are massless at the self-dual radius is crucial in obtaining radion stabilization in the Einstein frame (for more general studies of the importance of massless modes in string cosmology see [161, 162]). Such massless modes do not exist in all known string theories. They exist in the Heterotic theory, but not in Type II theories [142]. The following discussion is taken from [163].

Let us consider the equations of motion which arise from coupling the Einstein action to a string gas. In the anisotropic setting when the metric is taken to be

$$
d s^{2}=d t^{2}-a(t)^{2} d \mathbf{x}^{2}-\sum_{\alpha=1}^{6} b_{\alpha}(t)^{2} d y_{\alpha}^{2},
$$

where the $y_{\alpha}$ are the internal coordinates, the equation of motion for $b_{\alpha}$ becomes

$$
\ddot{b}_{\alpha}+\left(3 H+\sum_{\beta=1, \beta \neq \alpha}^{6} \frac{\dot{b}_{\beta}}{b_{\beta}}\right) \dot{b}_{\alpha}=\sum_{n, m} 8 \pi G \frac{\mu_{m, n}}{\sqrt{g} \varepsilon_{m, n}} \mathscr{S}
$$

where $\mu_{m, n}$ is the number density of $(m, n)$ strings, $\varepsilon_{m, n}$ is the energy of an individual $(m, n)$ string, and $g$ is the determinant of the metric. The source term $\mathscr{S}$ depends on the quantum numbers of the string gas, and the sum runs over all momentum and winding number vectors $m$ and $n$, respectively (note that $n$ and $m$ are six-vectors, one component for each internal dimension). If the number of right-moving oscillator modes is given by $N$, then the source term for fixed $m$ and $n$ vectors is

$$
\mathscr{S}=\sum_{\alpha}\left(\frac{m_{\alpha}}{b_{\alpha}}\right)^{2}-\sum_{\alpha} n_{\alpha}^{2} b_{\alpha}^{2}+\frac{2}{D-1}[(n, n)+(n, m)+2(N-1)] .
$$

To obtain this equation, we have made use of the mass spectrum of string states and of the level matching conditions. In the case of the bosonic superstring, the mass $M$ of a string state with fixed $m, n, N$ and $\tilde{N}$, where $N$ and $\tilde{N}$ are the number of right- and left-moving oscillator states, on a six-dimensional torus whose radii are given by $b_{\alpha}$ is

$$
M^{2}=\left(\frac{m_{\alpha}}{b_{\alpha}}\right)^{2}-\sum_{\alpha} n_{\alpha}^{2} b_{\alpha}^{2}+2(N+\tilde{N}-2),
$$

and the level matching condition reads

$$
\tilde{N}=(n, m)+N
$$

where $(n, m)$ indicates the scalar product of $n$ and $m$ in the trivial metric.

There are modes which are massless at the self-dual radius $b_{\alpha}=1$. One such mode is the graviton with $n=m=0$ and $N=1$. The modes of interest to us are modes which contain winding and momentum, namely

- $N=1,(m, m)=1,(m, n)=-1$ and $(n, n)=1$;

- $N=0,(m, m)=1,(m, n)=1$ and $(n, n)=1$;

- $N=0(m, m)=2,(m, n)=0$ and $(n, n)=2$. 
Note that these modes survive in the Heterotic string theory, but do not survive the GSO [142] truncation in Type II string theories.

In string theories which admit massless states (i.e. states which are massless at the self-dual radius), these states will dominate the initial partition function. The background dynamics will then also be dominated by these states. To understand the effect of these strings, consider the equation of motion (5.6) with the source term (5.7). The first two terms in the source term correspond to an effective potential with a stable minimum at the self-dual radius. However, if the third term in the source does not vanish at the self-dual radius, it will lead to a positive potential which causes the radion to increase. Thus, a condition for the stabilization of $b_{\alpha}$ at the self-dual radius is that the third term in (5.7) vanishes at the self-dual radius. This is the case if and only if the string state is a massless mode.

The massless modes have other nice features which are explored in detail in [160]. They act as radiation from the point of view of our three large dimensions and hence do not lead to a over-abundance problem. As our three spatial dimensions grow, the potential which confines the radion becomes shallower. However, rather surprisingly, it turns out the the potential remains steep enough to avoid fifth force constraints.

In the presence of massless string states, the shape moduli also can be stabilized, at least in the simple toroidal backgrounds considered so far [164]. To study this issue, we consider a metric of the form

$$
d s^{2}=d t^{2}-d \mathbf{x}^{2}-G_{m n} d y^{m} d y^{n},
$$

where the metric of the internal space (here for simplicity considered to be a two-dimensional torus) contains a shape modulus, the angle between the two cycles of the torus:

$$
G_{11}=G_{22}=1
$$

and

$$
G_{12}=G_{21}=\sin \theta,
$$

where $\theta=0$ corresponds to a rectangular torus. The ratio between the two toroidal radii is a second shape modulus. However, from the discussion of the previous subsection we already know that each radion individually is stabilized at the self-dual radius. Thus, the shape modulus corresponding to the ratio of the toroidal radii is fixed, and the angle is the only shape modulus which is yet to be considered.

Combining the 00 and the 12 Einstein equations, we obtain a harmonic oscillator equation for $\theta$ with $\theta=0$ as the stable fixed point.

$$
\ddot{\theta}+8 K^{-1 / 2} e^{-2 \phi} \theta=0,
$$

where $K$ is a constant whose value depends on the quantum numbers of the string gas. In the case of an expanding three-dimensional space we would have obtained an additional damping term in the above equation of motion. We thus conclude that the shape modulus is dynamically stabilized at a value which maximizes the area to circumference ratio.

The only modulus which is not stabilized by string winding and momentum modes is the dilaton. Recently, it has been show [165] that a gaugino condensation mechanism (similar to those 
used in string inflation model building) can be introduced which generates a stabilizing potential for the dilaton without interfering with the radion stabilization force provided by the string winding and momentum modes.

In the next subsection we turn to the predictions of string gas cosmology for observations. These predictions do not depend on the details of the theory, but only on three inputs. The first is the existence of a quasi-static initial phase in thermal equilibrium. The second condition is the applicability of the Einstein field equations for fluctuations on infrared scales (scales of the order of $1 \mathrm{~mm}$ ), many orders of magnitude larger than the string scale, and the third is a holographic scaling of the specific heat capacity. The role of the last condition will become manifest below.

\subsection{Fluctuations in String Gas Cosmology}

\subsubsection{Overview}

At the outset of this section, let us recall the mechanism by which inflationary cosmology leads to the possibility of a causal generation mechanism for cosmological fluctuations which yields an almost scale-invariant spectrum of perturbations. The space-time diagram of inflationary cosmology is sketched in Figure 5.

During the period of inflation, the Hubble radius

$$
l_{H}(t)=\frac{a}{\dot{a}}
$$

is approximately constant. In contrast, the physical length of a fixed co-moving scale (labelled by $k$ in the figure) is expanding exponentially. In this way, in inflationary cosmology scales which have microscopic sub-Hubble wavelengths at the beginning of inflation are red-shifted to become superHubble-scale fluctuations at the end of the period of inflation. After inflation, the Hubble radius increases linearly in time, faster than the physical wavelength corresponding to a fixed co-moving scale. Thus, scales re-enter the Hubble radius at late times.

Since inflation red-shifts any classical fluctuations which might have been present at the beginning of the inflationary phase, fluctuations in inflationary cosmology are generated by quantum vacuum perturbations. The fluctuations begin in their quantum vacuum state at the onset of inflation. Once the wavelength exceeds the Hubble radius, squeezing of the wave-function of the fluctuations sets in (see $[34,33]$ ). This squeezing plus the de-coherence of the fluctuations due to the interaction between short and long wavelength modes generated by the intrinsic non-linearities in both the gravitational and matter sectors of the theory (see $[71,70]$ for recent discussions of this aspect and references to previous work) lead to the classicalization of the fluctuations on superHubble scales.

Let us now turn to the cosmological background of string gas cosmology represented in Figure 13. This string gas cosmology background yields the space-time diagram sketched in Figure 15. As in Figure 5, the vertical axis is time and the horizontal axis denotes the physical distance. For times $t<t_{R}$, we are in the static Hagedorn phase and the Hubble radius is infinite. For $t>t_{R}$, the Einstein frame Hubble radius is expanding as in standard cosmology. The time $t_{R}$ is when the string winding modes begin to decay into string loops, and the scale factor starts to increase, leading to the transition to the radiation phase of standard cosmology. 


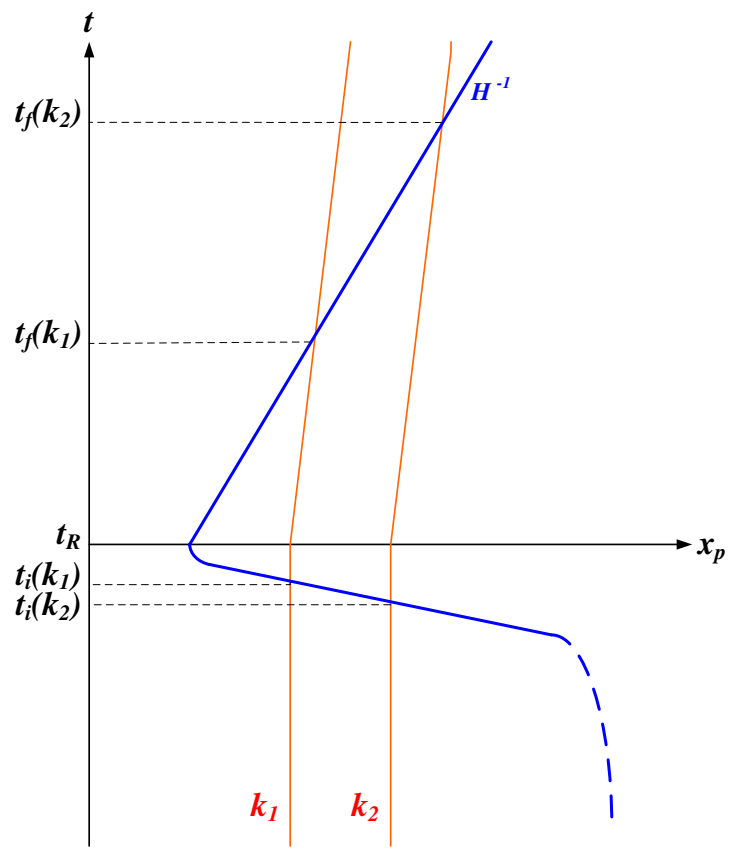

Figure 15: Space-time diagram (sketch) showing the evolution of fixed co-moving scales in string gas cosmology. The vertical axis is time, the horizontal axis is physical distance. The solid curve represents the Einstein frame Hubble radius $H^{-1}$ which shrinks abruptly to a micro-physical scale at $t_{R}$ and then increases linearly in time for $t>t_{R}$. Fixed co-moving scales (the dotted lines labeled by $k_{1}$ and $k_{2}$ ) which are currently probed in cosmological observations have wavelengths which are smaller than the Hubble radius before $t_{R}$. They exit the Hubble radius at times $t_{i}(k)$ just prior to $t_{R}$, and propagate with a wavelength larger than the Hubble radius until they reenter the Hubble radius at times $t_{f}(k)$.

Let us now compare the evolution of the physical wavelength corresponding to a fixed comoving scale with that of the Einstein frame Hubble radius $H^{-1}(t)$. The evolution of scales in string gas cosmology is identical to the evolution in standard and in inflationary cosmology for $t>t_{R}$. If we follow the physical wavelength of the co-moving scale which corresponds to the current Hubble radius back to the time $t_{R}$, then - taking the Hagedorn temperature to be of the order $10^{16} \mathrm{GeV}$ - we obtain a length of about $1 \mathrm{~mm}$. Compared to the string scale and the Planck scale, this is in the far infrared.

The physical wavelength is constant in the Hagedorn phase since space is static. But, as we enter the Hagedorn phase going back in time, the Hubble radius diverges to infinity. Hence, as in the case of inflationary cosmology, fluctuation modes begin sub-Hubble during the Hagedorn phase, and thus a causal generation mechanism for fluctuations is possible.

However, the physics of the generation mechanism is very different. In the case of inflationary cosmology, fluctuations are assumed to start as quantum vacuum perturbations because classical 
inhomogeneities are red-shifting. In contrast, in the Hagedorn phase of string gas cosmology there is no red-shifting of classical matter. Hence, it is the fluctuations in the classical matter which dominate. Since classical matter is a string gas, the dominant fluctuations are string thermodynamic fluctuations.

Our proposal for string gas structure formation is the following [31] (see [166] for a more detailed description). For a fixed co-moving scale with wavenumber $k$ we compute the matter fluctuations while the scale in sub-Hubble (and therefore gravitational effects are sub-dominant). When the scale exits the Hubble radius at time $t_{i}(k)$ we use the gravitational constraint equations to determine the induced metric fluctuations, which are then propagated to late times using the usual equations of gravitational perturbation theory. Since the scales we are interested in are in the far infrared, we use the Einstein constraint equations for fluctuations.

\subsubsection{Spectrum of Cosmological Fluctuations}

We write the metric including cosmological perturbations (scalar metric fluctuations) and gravitational waves in the following form (using conformal time $\eta$ )

$$
d s^{2}=a^{2}(\eta)\left\{(1+2 \Phi) d \eta^{2}-\left[(1-2 \Phi) \delta_{i j}+h_{i j}\right] d x^{i} d x^{j}\right\}
$$

We have fixed the gauge (i.e. coordinate) freedom for the scalar metric perturbations by adopting the longitudinal gauge and we have taken matter to be free of anisotropic stress. The spatial tensor $h_{i j}(\mathbf{x}, t)$ is transverse and traceless and represents the gravitational waves.

Note that in contrast to the case of slow-roll inflation, scalar metric fluctuations and gravitational waves are generated by matter at the same order in cosmological perturbation theory. This could lead to the expectation that the amplitude of gravitational waves in string gas cosmology should be generically larger than in inflationary cosmology. This expectation, however, is not realized [167] since there is a different mechanism which suppresses the gravitational waves relative to the density perturbations (namely the fact that the gravitational wave amplitude is set by the amplitude of the pressure, and the pressure is suppressed relative to the energy density in the Hagedorn phase).

Assuming that the fluctuations are described by the perturbed Einstein equations (they are not if the dilaton is not fixed $[168,169])$, then the spectra of cosmological perturbations $\Phi$ and gravitational waves $h$ are given by the energy-momentum fluctuations in the following way [166]

$$
\left\langle|\Phi(k)|^{2}\right\rangle=16 \pi^{2} G^{2} k^{-4}\left\langle\delta T^{0}{ }_{0}(k) \delta T^{0}{ }_{0}(k)\right\rangle,
$$

where the pointed brackets indicate expectation values, and

$$
\left\langle|h(k)|^{2}\right\rangle=16 \pi^{2} G^{2} k^{-4}\left\langle\delta T^{i}{ }_{j}(k) \delta T^{i}{ }_{j}(k)\right\rangle,
$$

where on the right hand side of (5.17) we mean the average over the correlation functions with $i \neq j$, and $h$ is the amplitude of the gravitational waves ${ }^{14}$.

Let us now use (5.16) to determine the spectrum of scalar metric fluctuations. We first calculate the root mean square energy density fluctuations in a sphere of radius $R=k^{-1}$. For a system in

\footnotetext{
${ }^{14}$ The gravitational wave tensor $h_{i j}$ can be written as the amplitude $h$ multiplied by a constant polarization tensor.
} 
thermal equilibrium they are given by the specific heat capacity $C_{V}$ via

$$
\left\langle\delta \rho^{2}\right\rangle=\frac{T^{2}}{R^{6}} C_{V} .
$$

The specific heat of a gas of closed strings on a torus of radius $R$ can be derived from the partition function of a gas of closed strings. This computation was carried out in [170] with the result

$$
C_{V} \approx 2 \frac{R^{2} / \ell^{3}}{T\left(1-T / T_{H}\right)}
$$

The specific heat capacity scales holographically with the size of the box. This result follows rigorously from evaluating the string partition function in the Hagedorn phase. The result, however, can also be understood heuristically: in the Hagedorn phase the string winding modes are crucial. These modes look like point particles in one less spatial dimension. Hence, we expect the specific heat capacity to scale like in the case of point particles in one less dimension of space ${ }^{15}$.

With these results, the power spectrum $P(k)$ of scalar metric fluctuations can be evaluated as follows

$$
\begin{aligned}
P_{\Phi}(k) & \equiv \frac{1}{2 \pi^{2}} k^{3}|\Phi(k)|^{2} \\
& =8 G^{2} k^{-1}<|\delta \rho(k)|^{2}>. \\
& =8 G^{2} k^{2}<(\delta M)^{2}>_{R} \\
& =8 G^{2} k^{-4}<(\delta \rho)^{2}>_{R} \\
& =8 G^{2} \frac{T}{\ell_{S}^{3}} \frac{1}{1-T / T_{H}},
\end{aligned}
$$

where in the first step we have used (5.16) to replace the expectation value of $|\Phi(k)|^{2}$ in terms of the correlation function of the energy density, and in the second step we have made the transition to position space

The first conclusion from the result (5.20) is that the spectrum is approximately scale-invariant $\left(P(k)\right.$ is independent of $k$ ). It is the 'holographic' scaling $C_{V}(R) \sim R^{2}$ which is responsible for the overall scale-invariance of the spectrum of cosmological perturbations. However, there is a small $k$ dependence which comes from the fact that in the above equation for a scale $k$ the temperature $T$ is to be evaluated at the time $t_{i}(k)$. Thus, the factor $\left(1-T / T_{H}\right)$ in the denominator is responsible for giving the spectrum a slight dependence on $k$. Since the temperature slightly decreases as time increases at the end of the Hagedorn phase, shorter wavelengths for which $t_{i}(k)$ occurs later obtain a smaller amplitude. Thus, the spectrum has a slight red tilt.

\subsubsection{Key Prediction of String Gas Cosmology}

As discovered in [167], the spectrum of gravitational waves is also nearly scale invariant. However, in the expression for the spectrum of gravitational waves the factor $\left(1-T / T_{H}\right)$ appears in the numerator, thus leading to a slight blue tilt in the spectrum. This is a prediction with which the cosmological effects of string gas cosmology can be distinguished from those of inflationary

\footnotetext{
${ }^{15}$ We emphasize that it was important for us to have compact spatial dimensions in order to obtain the winding modes which are crucial to get the holographic scaling of the thermodynamic quantities.
} 
cosmology, where quite generically a slight red tilt for gravitational waves results. The physical reason is that large scales exit the Hubble radius earlier when the pressure and hence also the off-diagonal spatial components of $T_{\mu \nu}$ are closer to zero.

Let us analyze this issue in a bit more detail and estimate the dimensionless power spectrum of gravitational waves. First, we make some general comments. In slow-roll inflation, to leading order in perturbation theory matter fluctuations do not couple to tensor modes. This is due to the fact that the matter background field is slowly evolving in time and the leading order gravitational fluctuations are linear in the matter fluctuations. In our case, the background is not evolving (at least at the level of our computations), and hence the dominant metric fluctuations are quadratic in the matter field fluctuations. At this level, matter fluctuations induce both scalar and tensor metric fluctuations. Based on this consideration we might expect that in our string gas cosmology scenario, the ratio of tensor to scalar metric fluctuations will be larger than in simple slow-roll inflationary models. However, since the amplitude $h$ of the gravitational waves is proportional to the pressure, and the pressure is suppressed in the Hagedorn phase, the amplitude of the gravitational waves will also be small in string gas cosmology.

The method for calculating the spectrum of gravitational waves is similar to the procedure outlined in the last section for scalar metric fluctuations. For a mode with fixed co-moving wavenumber $k$, we compute the correlation function of the off-diagonal spatial elements of the string gas energy-momentum tensor at the time $t_{i}(k)$ when the mode exits the Hubble radius and use (5.17) to infer the amplitude of the power spectrum of gravitational waves at that time. We then follow the evolution of the gravitational wave power spectrum on super-Hubble scales for $t>t_{i}(k)$ using the equations of general relativistic perturbation theory.

The power spectrum of the tensor modes is given by (5.17):

$$
P_{h}(k)=16 \pi^{2} G^{2} k^{-4} k^{3}\left\langle\delta T^{i}{ }_{j}(k) \delta T^{i}{ }_{j}(k)\right\rangle
$$

for $i \neq j$. Note that the $k^{3}$ factor multiplying the momentum space correlation function of $T^{i}{ }_{j}$ gives the position space correlation function $C^{i}{ }_{j}{ }^{i}{ }_{j}(R)$, namely the root mean square fluctuation of $T^{i}{ }_{j}$ in a region of radius $R=k^{-1}$ (the reader who is skeptical about this point is invited to check that the dimensions work out properly). Thus,

$$
P_{h}(k)=16 \pi^{2} G^{2} k^{-4} C^{i}{ }_{j j}^{i}(R) .
$$

The correlation function $C^{i}{ }_{j}{ }_{j}$ on the right hand side of the above equation follows from the thermal closed string partition function and was computed explicitly in [171] (see also [11]). We obtain

$$
P_{h}(k) \sim 16 \pi^{2} G^{2} \frac{T}{l_{s}^{3}}\left(1-T / T_{H}\right) \ln ^{2}\left[\frac{1}{l_{s}^{2} k^{2}}\left(1-T / T_{H}\right)\right],
$$

which, for temperatures close to the Hagedorn value reduces to

$$
P_{h}(k) \sim\left(\frac{l_{P l}}{l_{s}}\right)^{4}\left(1-T / T_{H}\right) \ln ^{2}\left[\frac{1}{l_{s}^{2} k^{2}}\left(1-T / T_{H}\right)\right] .
$$

This shows that the spectrum of tensor modes is - to a first approximation, namely neglecting the logarithmic factor and neglecting the $k$-dependence of $T\left(t_{i}(k)\right)$ - scale-invariant. 
On super-Hubble scales, the amplitude $h$ of the gravitational waves is constant. The wave oscillations freeze out when the wavelength of the mode crosses the Hubble radius. As in the case of scalar metric fluctuations, the waves are squeezed. Whereas the wave amplitude remains constant, its time derivative decreases. Another way to see this squeezing is to change variables to

$$
\psi(\eta, \mathbf{x})=a(\eta) h(\eta, \mathbf{x})
$$

in terms of which the action has canonical kinetic term. The action in terms of $\psi$ becomes

$$
S=\frac{1}{2} \int d^{4} x\left(\psi^{\prime 2}-\psi_{, i} \psi_{, i}+\frac{a^{\prime \prime}}{a} \psi^{2}\right)
$$

from which it immediately follows that on super-Hubble scales $\psi \sim a$. This is the squeezing of gravitational waves [72].

Since there is no $k$-dependence in the squeezing factor, the scale-invariance of the spectrum at the end of the Hagedorn phase will lead to a scale-invariance of the spectrum at late times.

Note that in the case of string gas cosmology, the squeezing factor $z(\eta)$ does not differ substantially from the squeezing factor $a(\eta)$ for gravitational waves. In the case of inflationary cosmology, $z(\eta)$ and $a(\eta)$ differ greatly during reheating, leading to a much larger squeezing for scalar metric fluctuations, and hence to a suppressed tensor to scalar ratio of fluctuations. In the case of string gas cosmology, there is no difference in squeezing between the scalar and the tensor modes.

Let us return to the discussion of the spectrum of gravitational waves. The result for the power spectrum is given in (5.24), and we mentioned that to a first approximation this corresponds to a scale-invariant spectrum. As realized in [167], the logarithmic term and the $k$-dependence of $T\left(t_{i}(k)\right)$ both lead to a small blue-tilt of the spectrum. This feature is characteristic of our scenario and cannot be reproduced in inflationary models. In inflationary models, the amplitude of the gravitational waves is set by the Hubble constant $H$. The Hubble constant cannot increase during inflation, and hence no blue tilt of the gravitational wave spectrum is possible.

A heuristic way of understanding the origin of the slight blue tilt in the spectrum of tensor modes is as follows. The closer we get to the Hagedorn temperature, the more the thermal bath is dominated by long string states, and thus the smaller the pressure will be compared to the pressure of a pure radiation bath. Since the pressure terms (strictly speaking the anisotropic pressure terms) in the energy-momentum tensor are responsible for the tensor modes, we conclude that the smaller the value of the wavenumber $k$ (and thus the higher the temperature $T\left(t_{i}(k)\right)$ when the mode exits the Hubble radius, the lower the amplitude of the tensor modes. In contrast, the scalar modes are determined by the energy density, which increases at $T\left(t_{i}(k)\right)$ as $k$ decreases, leading to a slight red tilt.

The reader may ask about the predictions of string gas cosmology for non-Gaussianities. The answer is [172] that the non-Gaussianities from the thermal string gas perturbations are Poissonsuppressed on scales larger than the thermal wavelength in the Hagedorn phase. However, if the spatial sections are initially large, then it is possible that a network of cosmic superstrings [173] will be left behind. These strings - if stable - would achieve a scaling solution (constant number of strings crossing each Hubble volume at each time [86, 87, 88]). Such strings give rise to linear discontinuities in the CMB temperature maps [174], lines which can be searched for using edge detection algorithms such as the Canny algorithm (see [175] for recent feasibility studies). 


\subsection{Problems of String Gas Cosmology}

The main problem of the current implementation of string gas cosmology is that it does not provide a quantitative model for the Hagedorn phase. The criticisms of string gas cosmology raised in $[169,176]$ are based on assuming that dilaton gravity should be the background. However, as mentioned earlier, this is not a reasonable assumption since the coupling of string gas matter to dilaton gravity is not S-duality invariant. The quasi-static early Hagedorn phase should also have constant dilaton. In addition, we do not expect that at high densities such as the Hagedorn density an simple effective action such as that of dilaton gravity will apply. Nevertheless, to make the string gas cosmology scenario into a real theory, it is crucial to obtain a good understanding of the background dynamics. For some initial steps in this direction see [149].

A second problem of string gas cosmology is the size problem (and the related entropy problem). If the string scale is about $10^{17} \mathrm{GeV}$ as is preferred in early heterotic superstring models, then the radius of the universe during the Hagedorn phase must be many orders of magnitude larger than the string scale. Without embedding string gas cosmology into a bouncing cosmology, it seems unnatural to demand such a large initial size. This problem disappears if the Hagedorn phase is preceded by a phase of contraction, as in the model of [177]. In this case, however, it is non-trivial to arrange that the Hagedorn phase lasts sufficiently long to maintain thermal equilibrium over the required range of scales.

It should be noted, however, that some of the conceptual problems of inflationary cosmology such as the trans-Planckian problem for fluctuations, do not arise in string gas cosmology. As in the case of the matter bounce scenario, the basic mechanism of the scenario is insensitive to what sets the cosmological constant to its observed very small value.

\section{Conclusions}

In these lectures I have argued that it is possible to explore physics of the very early universe using current cosmological observations. Given the large amount of new data which is expected over the next decade, early universe cosmology will be a vibrant field of research. The information about the very early universe is transferred to the current time mainly via imprints on the spectrum of cosmological fluctuations. Thus, the theory of cosmological fluctuations is the key tool of modern cosmology. It links current observations with early universe cosmology. The theory can be applied to any background cosmology, not just inflationary cosmology. I have illustrated the application of the theory of cosmological perturbations to three scenarios of the very early universe, the inflationary scenario, the matter bounce paradigm, and string gas cosmology.

The Hubble radius plays a key role in the evolution of fluctuations. On sub-Hubble scales the microphysical forces dominate, whereas on super-Hubble scales matter forces freeze out and gravity dominates. In order to have a causal mechanism of structure formation, it is therefore crucial that scales of current interest in cosmology originate at very early times inside the Hubble radius, and that they then propagate over an extended period of time on super-Hubble scales. This propagation on super-Hubble scales is required in order to obtain the squeezing of the perturbations which is required to explain the acoustic oscillations in the angular power spectrum of CMB anisotropies.

The inflationary scenario is the current paradigm of early universe cosmology. It explains important conceptual problems of Standard Big Bang Cosmology, and most importantly it provided 
a mechanism to explain the origin of structure in the universe via causal physics. The perturbations begin as quantum vacuum fluctuations on sub-Hubble scales. They exit the Hubble radius during the phase of inflationary expansion of space and are then squeezed. Inflationary cosmology has been predictive: it predicted the detailed shape of the angular power spectrum of CMB anisotropies more than 15 years before the observations. However, it is important to realize that any theory which provides a scale-invariant spectrum of primordial curvature fluctuations and which admits a period during which the scales which are observed today evolve outside of the Hubble radius will have the same predictions, as already realized more than a decade before the development of inflationary cosmology by Sunyaev and Zel'dovich [178] and by Peebles and Yu [179] (see also $[36,37])$. Thus, it is incorrect to claim that the current observations confirm the inflationary scenario. In fact, current realizations of inflationary cosmology suffer from some basic conceptual problems. This motivates the search for an improved understanding of the very early universe.

I have described two alternative early universe scenarios, the "matter bounce", and "string gas cosmology". Both lead to a scale-invariant spectrum of curvature fluctuations and involve squeezing of the perturbations on super-Hubble scales and are thus in agreement with current observations. The challenge for alternative scenarios is to identify clean predictions with which the new scenarios can be distinguished from those of inflation. In the case of the matter bounce the prediction we have identified is a particular shape of the bispectrum, in the case of string gas cosmology the key prediction is a slight blue tilt in the spectrum of gravitational waves.

It is important to realize that physics of the very early universe is determined by physics of the highest energy scales, scales many orders of magnitude higher than those which are probed in accelerator experiments. Thus, a better understanding of quantum gravity (e.g. string theory) might lead to completely new possibilities for cosmology. In turn, it is only through cosmology that physics of the highest energies can be probed.

\section{Acknowledgments}

I wish to thank the organizers of this conference, in particular Maria Emilia Guimaraes, for the invitation to give these lectures and for their wonderful hospitality in Brazil. I wish to thank the students for their many good questions. This research is supported in part by a NSERC Discovery Grant, by the Canada Research Chairs program and by a Killam Foundation Research Fellowship.

\section{References}

[1] R. H. Brandenberger, “Cosmology of the Very Early Universe,” AIP Conf. Proc. 1268, 3-70 (2010). [arXiv:1003.1745 [hep-th]].

[2] Guth AH, "The Inflationary Universe: A Possible Solution To The Horizon And Flatness Problems," Phys. Rev. D 23, 347 (1981).

[3] R. Brout, F. Englert and E. Gunzig, "The Creation Of The Universe As A Quantum Phenomenon," Annals Phys. 115, 78 (1978).

[4] A. A. Starobinsky, “A New Type Of Isotropic Cosmological Models Without Singularity,” Phys. Lett. B 91, 99 (1980). 
[5] K. Sato, "First Order Phase Transition Of A Vacuum And Expansion Of The Universe," Mon. Not. Roy. Astron. Soc. 195, 467 (1981).

[6] V. Mukhanov and G. Chibisov, "Quantum Fluctuation And Nonsingular Universe. (In Russian)," JETP Lett. 33, 532 (1981) [Pisma Zh. Eksp. Teor. Fiz. 33, 549 (1981)].

[7] W. Press, "Spontaneous production of the Zel'dovich spectrum of cosmological fluctuations", Phys. Scr. 21, 702 (1980).

[8] A. A. Starobinsky, "Spectrum of relict gravitational radiation and the early state of the universe," JETP Lett. 30, 682 (1979) [Pisma Zh. Eksp. Teor. Fiz. 30, 719 (1979)].

[9] C. L. Bennett et al., "First Year Wilkinson Microwave Anisotropy Probe (WMAP) Observations: Preliminary Maps and Basic Results," Astrophys. J. Suppl. 148, 1 (2003) [arXiv:astro-ph/0302207].

[10] R. H. Brandenberger, “Alternatives to Cosmological Inflation,” arXiv:0902.4731 [hep-th].

[11] R. H. Brandenberger, “String Gas Cosmology,” arXiv:0808.0746 [hep-th].

[12] H. P. Gush, M. Halpern and E. H. Wishnow, "Rocket Measurement Of The Cosmic-Background-Radiation Mm-Wave Spectrum,” Phys. Rev. Lett. 65, 537 (1990);

J. C. Mather et al., "Measurement of the Cosmic Microwave Background spectrum by the COBE FIRAS instrument," Astrophys. J. 420, 439 (1994).

[13] D. Wands, "Duality invariance of cosmological perturbation spectra," Phys. Rev. D 60, 023507 (1999) [arXiv:gr-qc/9809062].

[14] F. Finelli and R. Brandenberger, "On the generation of a scale-invariant spectrum of adiabatic fluctuations in cosmological models with a contracting phase," Phys. Rev. D 65, 103522 (2002) [arXiv:hep-th/0112249].

[15] L. E. Allen and D. Wands, "Cosmological perturbations through a simple bounce," Phys. Rev. D 70, 063515 (2004) [arXiv:astro-ph/0404441].

[16] T. Biswas, A. Mazumdar and W. Siegel, “Bouncing universes in string-inspired gravity,” JCAP 0603, 009 (2006) [arXiv:hep-th/0508194].

[17] R. H. Brandenberger, V. F. Mukhanov and A. Sornborger, "A Cosmological theory without singularities,” Phys. Rev. D 48, 1629 (1993) [arXiv:gr-qc/9303001].

[18] R. Brandenberger, H. Firouzjahi and O. Saremi, "Cosmological Perturbations on a Bouncing Brane," JCAP 0711, 028 (2007) [arXiv:0707.4181 [hep-th]].

[19] Y. F. Cai, T. Qiu, Y. S. Piao, M. Li and X. Zhang, “Bouncing Universe with Quintom Matter," JHEP 0710, 071 (2007) [arXiv:0704.1090 [gr-qc]];

Y. F. Cai, T. T. Qiu, J. Q. Xia and X. Zhang, “A Model Of Inflationary Cosmology Without Singularity," arXiv:0808.0819 [astro-ph].

[20] M. Novello and S. E. P. Bergliaffa, “Bouncing Cosmologies,” Phys. Rept. 463, 127 (2008) [arXiv:0802.1634 [astro-ph]].

[21] R. Brandenberger, "Matter Bounce in Horava-Lifshitz Cosmology," Phys. Rev. D 80, 043516 (2009) [arXiv:0904.2835 [hep-th]].

[22] P. Horava, “Quantum Gravity at a Lifshitz Point,” Phys. Rev. D 79, 084008 (2009) [arXiv:0901.3775 [hep-th]].

[23] S. Alexander, T. Biswas and R. H. Brandenberger, "On the Transfer of Adiabatic Fluctuations through a Nonsingular Cosmological Bounce,” arXiv:0707.4679 [hep-th]. 
[24] Y. F. Cai, T. Qiu, R. Brandenberger, Y. S. Piao and X. Zhang, "On Perturbations of Quintom Bounce," JCAP 0803, 013 (2008) [arXiv:0711.2187 [hep-th]];

Y. F. Cai and X. Zhang, "Evolution of Metric Perturbations in Quintom Bounce model," arXiv:0808.2551 [astro-ph].

[25] Y. F. Cai, T. Qiu, R. Brandenberger and X. Zhang, "A Nonsingular Cosmology with a Scale-Invariant Spectrum of Cosmological Perturbations from Lee-Wick Theory," arXiv:0810.4677 [hep-th].

[26] X. Gao, Y. Wang, W. Xue and R. Brandenberger, "Fluctuations in a Hořava-Lifshitz Bouncing Cosmology," arXiv:0911.3196 [hep-th];

X. Gao, Y. Wang, R. Brandenberger and A. Riotto, "Cosmological Perturbations in Hořava-Lifshitz Gravity," arXiv:0905.3821 [hep-th].

[27] Y. F. Cai, W. Xue, R. Brandenberger and X. m. Zhang, "Thermal Fluctuations and Bouncing Cosmologies," JCAP 0906, 037 (2009) [arXiv:0903.4938 [hep-th]].

[28] R. H. Brandenberger and C. Vafa, "Superstrings in the Early Universe," Nucl. Phys. B 316, 391 (1989).

[29] J. Kripfganz and H. Perlt, "Cosmological Impact Of Winding Strings,” Class. Quant. Grav. 5, 453 (1988).

[30] R. Hagedorn, "Statistical Thermodynamics Of Strong Interactions At High-Energies," Nuovo Cim. Suppl. 3, 147 (1965).

[31] A. Nayeri, R. H. Brandenberger and C. Vafa, "Producing a scale-invariant spectrum of perturbations in a Hagedorn phase of string cosmology," Phys. Rev. Lett. 97, 021302 (2006) [arXiv:hep-th/0511140].

[32] R. H. Brandenberger, "Processing of Cosmological Perturbations in a Cyclic Cosmology," Phys. Rev. D 80, 023535 (2009) [arXiv:0905.1514 [hep-th]].

[33] R. H. Brandenberger, "Lectures on the theory of cosmological perturbations," Lect. Notes Phys. 646, 127 (2004) [arXiv:hep-th/0306071].

[34] V. F. Mukhanov, H. A. Feldman and R. H. Brandenberger, "Theory of cosmological perturbations. Part 1. Classical perturbations. Part 2. Quantum theory of perturbations. Part 3. Extensions," Phys. Rept. 215, 203 (1992).

[35] R. K. Sachs and A. M. Wolfe, "Perturbations of a cosmological model and angular variations of the microwave background," Astrophys. J. 147, 73 (1967) [Gen. Rel. Grav. 39, 1929 (2007)].

[36] E. R. Harrison, "Fluctuations at the threshold of classical cosmology," Phys. Rev. D 1, 2726 (1970).

[37] Y. B. Zeldovich, “A Hypothesis, unifying the structure and the entropy of the universe," Mon. Not. Roy. Astron. Soc. 160, 1P (1972).

[38] N. Afshordi and R. H. Brandenberger, "Super-Hubble nonlinear perturbations during inflation," Phys. Rev. D 63, 123505 (2001) [arXiv:gr-qc/0011075].

[39] E. Lifshitz, “On the Gravitational stability of the expanding universe," J. Phys. (USSR) 10, 116 (1946);

E. M. Lifshitz and I. M. Khalatnikov, "Investigations in relativistic cosmology,” Adv. Phys. 12, 185 (1963).

[40] J. M. Bardeen, “Gauge Invariant Cosmological Perturbations,” Phys. Rev. D 22, 1882 (1980).

[41] W. H. Press and E. T. Vishniac, "Tenacious myths about cosmological perturbations larger than the horizon size,” Astrophys. J. 239, 1 (1980). 
[42] R. H. Brandenberger, R. Kahn and W. H. Press, "Cosmological Perturbations In The Early Universe," Phys. Rev. D 28, 1809 (1983).

[43] H. Kodama and M. Sasaki, “Cosmological Perturbation Theory,” Prog. Theor. Phys. Suppl. 78, 1 (1984).

[44] M. Bruni, G. F. R. Ellis and P. K. S. Dunsby, "Gauge invariant perturbations in a scalar field dominated universe,” Class. Quant. Grav. 9, 921 (1992).

[45] J. c. Hwang, "Evolution of ideal fluid cosmological perturbations," Astrophys. J. 415, 486 (1993).

[46] R. Durrer, “Anisotropies in the cosmic microwave background: Theoretical foundations,” Helv. Phys. Acta 69, 417 (1996) [arXiv:astro-ph/9610234].

[47] J. M. Stewart, "Perturbations of Friedmann-Robertson-Walker cosmological models," Class. Quant. Grav. 7, 1169 (1990).

[48] N. Turok, U. L. Pen and U. Seljak, "The scalar, vector and tensor contributions to CMB anisotropies from cosmic defects,” Phys. Rev. D 58, 023506 (1998) [arXiv:astro-ph/9706250].

[49] T. J. Battefeld and R. Brandenberger, "Vector perturbations in a contracting universe," Phys. Rev. D 70, 121302 (2004) [arXiv:hep-th/0406180].

[50] J. M. Stewart and M. Walker, "Perturbations Of Spacetimes In General Relativity,” Proc. Roy. Soc. Lond. A 341, 49 (1974).

[51] J. M. Bardeen, P. J. Steinhardt and M. S. Turner, "Spontaneous Creation Of Almost Scale - Free Density Perturbations In An Inflationary Universe,” Phys. Rev. D 28, 679 (1983).

[52] R. H. Brandenberger and R. Kahn, "Cosmological Perturbations In Inflationary Universe Models," Phys. Rev. D 29, 2172 (1984).

[53] D. H. Lyth, “Large Scale Energy Density Perturbations And Inflation,” Phys. Rev. D 31, 1792 (1985).

[54] F. Finelli and R. H. Brandenberger, "Parametric amplification of gravitational fluctuations during reheating,” Phys. Rev. Lett. 82, 1362 (1999) [arXiv:hep-ph/9809490].

[55] S. Weinberg, “Adiabatic modes in cosmology,” Phys. Rev. D 67, 123504 (2003) [arXiv:astro-ph/0302326].

[56] W. B. Lin, X. H. Meng and X. M. Zhang, "Adiabatic gravitational perturbation during reheating," Phys. Rev. D 61, 121301 (2000) [arXiv:hep-ph/9912510].

[57] B. A. Bassett and F. Viniegra, "Massless metric preheating," Phys. Rev. D 62, 043507 (2000) [arXiv:hep-ph/9909353].

[58] F. Finelli and R. H. Brandenberger, "Parametric amplification of metric fluctuations during reheating in two field models," Phys. Rev. D 62, 083502 (2000) [arXiv:hep-ph/0003172].

[59] J. H. Traschen, “Constraints On Stress Energy Perturbations In General Relativity,” Phys. Rev. D 31, 283 (1985).

[60] J. H. Traschen, N. Turok and R. H. Brandenberger, "Microwave Anisotropies from Cosmic Strings," Phys. Rev. D 34, 919 (1986).

[61] G. Dvali, A. Gruzinov and M. Zaldarriaga, "Cosmological perturbations from inhomogeneous reheating, freezeout, and mass domination,” Phys. Rev. D 69, 083505 (2004) [arXiv:astro-ph/0305548]. 
[62] L. Kofman, "Probing string theory with modulated cosmological fluctuations," arXiv:astro-ph/0303614.

[63] F. Vernizzi, “Cosmological perturbations from varying masses and couplings,” Phys. Rev. D 69, 083526 (2004) [arXiv:astro-ph/0311167].

[64] M. Axenides, R. H. Brandenberger and M. S. Turner, "Development Of Axion Perturbations In An Axion Dominated Universe,” Phys. Lett. B 126, 178 (1983).

[65] V. F. Mukhanov, "Quantum Theory of Gauge Invariant Cosmological Perturbations," Sov. Phys. JETP 67, 1297 (1988) [Zh. Eksp. Teor. Fiz. 94N7, 1 (1988)].

[66] V. F. Mukhanov, "Gravitational Instability Of The Universe Filled With A Scalar Field," JETP Lett. 41, 493 (1985) [Pisma Zh. Eksp. Teor. Fiz. 41, 402 (1985)].

[67] M. Sasaki, “Large Scale Quantum Fluctuations in the Inflationary Universe,” Prog. Theor. Phys. 76, 1036 (1986).

[68] V. N. Lukash, "Production of phonons in an isotropic universe," Sov. Phys. JETP 52, 807 (1980) [Zh. Eksp. Teor. Fiz. 79, (19??)].

[69] N. D. Birrell and P. C. W. Davies, “Quantum Fields In Curved Space,” Cambridge, Uk: Univ. Pr. ( 1982) $340 p$

[70] C. Kiefer, I. Lohmar, D. Polarski and A. A. Starobinsky, "Pointer states for primordial fluctuations in inflationary cosmology,” Class. Quant. Grav. 24, 1699 (2007) [arXiv:astro-ph/0610700].

[71] P. Martineau, "On the decoherence of primordial fluctuations during inflation," Class. Quant. Grav. 24, 5817 (2007) [arXiv:astro-ph/0601134].

[72] L. P. Grishchuk, “Amplification Of Gravitational Waves In An Istropic Universe,” Sov. Phys. JETP 40, 409 (1975) [Zh. Eksp. Teor. Fiz. 67, 825 (1974)].

[73] E. Silverstein and D. Tong, "Scalar Speed Limits and Cosmology: Acceleration from D-cceleration," Phys. Rev. D 70, 103505 (2004) [arXiv:hep-th/0310221].

[74] C. Armendariz-Picon, T. Damour and V. F. Mukhanov, “k-Inflation,” Phys. Lett. B 458, 209 (1999) [arXiv:hep-th/9904075].

[75] R. H. Brandenberger and J. H. Kung, "Chaotic Inflation As An Attractor In Initial Condition Space," Phys. Rev. D 42, 1008 (1990).

[76] S. R. Coleman, “The Fate Of The False Vacuum. 1. Semiclassical Theory,” Phys. Rev. D 15, 2929 (1977) [Erratum-ibid. D 16, 1248 (1977)].

[77] R. H. Brandenberger, “Quantum Field Theory Methods And Inflationary Universe Models," Rev. Mod. Phys. 57, 1 (1985).

[78] A. Linde, "A New Inflationary Universe Scenario: A Possible Solution Of The Horizon, Flatness, Homogeneity, Isotropy And Primordial Monopole Problems,” Phys. Lett. B 108, 389 (1982);

A. Albrecht and P. Steinhardt, "Cosmology For Grand Unified Theories With Radiatively Induced Symmetry Breaking,” Phys. Rev. Lett. 48, 1220 (1982).

[79] S. Coleman and E. Weinberg, "Radiative Corrections As The Origin Of Spontaneous Symmetry Breaking,” Phys. Rev. D 7, 1888 (1973).

[80] D. Goldwirth and T. Piran, "Initial conditions for inflation,” Phys. Rept. 214, 223 (1992);

A. Albrecht and R. Brandenberger, “On The Realization Of New Inflation,” Phys. Rev. D 31, 1225 (1985). 
[81] R. Brandenberger, G. Geshnizjani and S. Watson, "On the initial conditions for brane inflation," Phys. Rev. D 67, 123510 (2003) [arXiv:hep-th/0302222].

[82] G. F. Mazenko, R. M. Wald and W. G. Unruh, "Does A Phase Transition In The Early Universe Produce The Conditions Needed For Inflation?,” Phys. Rev. D 31, 273 (1985).

[83] A. Linde, “Chaotic Inflation,” Phys. Lett. B 129, 177 (1983).

[84] H. Feldman and R. Brandenberger, "Chaotic Inflation With Metric And Matter Perturbations," Phys. Lett. B 227, 359 (1989).

[85] A. Linde, “Hybrid inflation,” Phys. Rev. D 49, 748 (1994).

[86] A. Vilenkin and E.P.S. Shellard, Cosmic Strings and other Topological Defects (Cambridge Univ. Press, Cambridge, 1994).

[87] M. B. Hindmarsh and T. W. B. Kibble, “Cosmic strings,” Rept. Prog. Phys. 58, 477 (1995) [arXiv:hep-ph/9411342].

[88] R. H. Brandenberger, “Topological defects and structure formation,” Int. J. Mod. Phys. A 9, 2117 (1994) [arXiv:astro-ph/9310041].

[89] R. Allahverdi, R. Brandenberger, F. Y. Cyr-Racine and A. Mazumdar, "Reheating in Inflationary Cosmology: Theory and Applications,” Ann. Rev. Nucl. Part. Science 60, 27 (2010) [arXiv:1001.2600 [hep-th]].

[90] L. F. Abbott, E. Farhi and M. Wise, "Particle Production In The New Inflationary Cosmology," Phys. Lett. B 117, 29 (1982);

A. Dolgov and A. Linde, "Baryon Asymmetry In Inflationary Universe," Phys. Lett. B 116, 329 (1982);

A. Albrecht, P. J. Steinhardt, M. S. Turner and F. Wilczek, "Reheating An Inflationary Universe," Phys. Rev. Lett. 48, 1437 (1982).

[91] J. Traschen and R. Brandenberger, "Particle Production During Out-Of-Equilibrium Phase Transitions,” Phys. Rev. D 42, 2491 (1990).

[92] L. Kofman, A. Linde and A. Starobinsky, "Reheating after inflation,” Phys. Rev. Lett. 73, 3195 (1994).

[93] Y. Shtanov, J. Traschen and R. Brandenberger, "Universe reheating after inflation,” Phys. Rev. D 51, 5438 (1995); Shtanov, Y., Ukr. Fiz. Zh. 38, 1425 (1993).

[94] L. Kofman, A. Linde and A. Starobinsky, “Towards the theory of reheating after inflation," Phys. Rev. D 56, 3258 (1997).

[95] M. Gleiser and R. Ramos, "Microphysical approach to nonequilibrium dynamics of quantum fields," Phys. Rev. D 50, 2441 (1994).

[96] A. Dolgov and D. Kirilova, "Production of particles by a variable scalar field," Sov. J. Nucl. Phys. 51, 172 (1990) [Yad. Fiz. 51, 273 (1990)].

[97] N. W. McLachlan, "Theory and Applications of Mathieu Functions" (Oxford Univ. Press, Clarendon, 1947).

[98] B. Bassett, D. Kaiser and R. Maartens, “General relativistic preheating after inflation,” Phys. Lett. B 455, 84 (1999).

[99] C. Gordon, D. Wands, B. A. Bassett and R. Maartens, "Adiabatic and entropy perturbations from inflation,” Phys. Rev. D 63, 023506 (2001) [arXiv:astro-ph/0009131]. 
[100] R. Brandenberger, A. Frey and L. Lorenz, "Entropy Fluctuations in Brane Inflation Models," Int. J. Mod. Phys. A 24, 4327 (2009).

[101] R. Brandenberger, K. Dasgupta and A.-C. Davis, "A Study of Structure Formation and Reheating in the D3/D7 Brane Inflation Model,” Phys. Rev. D 78, 083502 (2008).

[102] L. P. Levasseur, G. Laporte and R. Brandenberger, “Analytical Study of Mode Coupling in Hybrid Inflation,” Phys. Rev. D 82, 123524 (2010). arXiv:1004.1425 [hep-th].

[103] F.-Y. Cyr-Racine and R. Brandenberger, "Study of the Growth of Entropy Modes in MSSM Flat Directions Decay: Constraints on the Parameter Space,” JCAP 0902, 022 (2009).

[104] F. L. Bezrukov and M. Shaposhnikov, “The Standard Model Higgs boson as the inflaton,” Phys. Lett. B 659, 703 (2008) [arXiv:0710.3755 [hep-th]].

[105] F. C. Adams, K. Freese and A. H. Guth, "Constraints on the scalar field potential in inflationary models," Phys. Rev. D 43, 965 (1991).

[106] R. H. Brandenberger, "Inflationary cosmology: Progress and problems," arXiv:hep-ph/9910410.

[107] R. H. Brandenberger and J. Martin, "The Robustness of inflation to changes in superPlanck scale physics,” Mod. Phys. Lett. A 16, 999 (2001), [arXiv:astro-ph/0005432];

J. Martin and R. H. Brandenberger, "The TransPlanckian problem of inflationary cosmology," Phys. Rev. D 63, 123501 (2001), [arXiv:hep-th/0005209].

[108] J. C. Niemeyer, "Inflation with a high frequency cutoff," Phys. Rev. D 63, 123502 (2001), [arXiv:astro-ph/0005533];

J. C. Niemeyer and R. Parentani, "Minimal modifications of the primordial power spectrum from an adiabatic short distance cutoff," Phys. Rev. D 64, 101301 (2001), [arXiv:astro-ph/0101451];

S. Shankaranarayanan, "Is there an imprint of Planck scale physics on inflationary cosmology?," Class. Quant. Grav. 20, 75 (2003) [arXiv:gr-qc/0203060].

[109] W. G. Unruh, "Sonic Analog Of Black Holes And The Effects Of High Frequencies On Black Hole Evaporation,” Phys. Rev. D 51, 2827 (1995).

[110] S. Corley and T. Jacobson, "Hawking Spectrum and High Frequency Dispersion,” Phys. Rev. D 54, 1568 (1996) [arXiv:hep-th/9601073].

[111] R. H. Brandenberger and J. Martin, "Back-reaction and the trans-Planckian problem of inflation revisited,” Phys. Rev. D 71, 023504 (2005) [arXiv:hep-th/0410223].

[112] T. Tanaka, "A comment on trans-Planckian physics in inflationary universe," arXiv:astro-ph/0012431.

[113] A. A. Starobinsky, "Robustness of the inflationary perturbation spectrum to trans-Planckian physics," Pisma Zh. Eksp. Teor. Fiz. 73, 415 (2001), [JETP Lett. 73, 371 (2001)], [arXiv:astro-ph/0104043].

[114] S. W. Hawking and G. F. R. Ellis, “The Large scale structure of space-time," Cambridge University Press, Cambridge, 1973

[115] A. Borde and A. Vilenkin, "Eternal inflation and the initial singularity," Phys. Rev. Lett. 72, 3305 (1994) [arXiv:gr-qc/9312022].

[116] R. H. Brandenberger, "Back reaction of cosmological perturbations and the cosmological constant problem," arXiv:hep-th/0210165.

[117] M. Gasperini and G. Veneziano, "Pre - big bang in string cosmology," Astropart. Phys. 1, 317 (1993) [arXiv:hep-th/9211021]. 
[118] J. Khoury, B. A. Ovrut, P. J. Steinhardt and N. Turok, "The ekpyrotic universe: Colliding branes and the origin of the hot big bang," Phys. Rev. D 64, 123522 (2001) [arXiv:hep-th/0103239].

[119] J. M. Cline, S. Jeon and G. D. Moore, "The phantom menaced: Constraints on low-energy effective ghosts,” Phys. Rev. D 70, 043543 (2004) [arXiv:hep-ph/0311312].

[120] B. Feng, X. L. Wang and X. M. Zhang, "Dark Energy Constraints from the Cosmic Age and Supernova,” Phys. Lett. B 607, 35 (2005) [arXiv:astro-ph/0404224];

B. Feng, M. Li, Y. S. Piao and X. Zhang, “Oscillating quintom and the recurrent universe," Phys. Lett. B 634, 101 (2006) [arXiv:astro-ph/0407432].

[121] B. Grinstein, D. O'Connell and M. B. Wise, “The Lee-Wick standard model,” Phys. Rev. D 77, 025012 (2008) [arXiv:0704.1845 [hep-ph]].

[122] J. Karouby and R. Brandenberger, “A Radiation Bounce from the Lee-Wick Construction?,” Phys. Rev. D 82, 063532 (2010) [arXiv:1004.4947 [hep-th]].

[123] C. Lin, R. H. Brandenberger and L. P. Levasseur, "A Matter Bounce By Means of Ghost Condensation,” arXiv:1007.2654 [hep-th].

[124] N. Arkani-Hamed, H. C. Cheng, M. A. Luty and S. Mukohyama, "Ghost condensation and a consistent infrared modification of gravity,” JHEP 0405, 074 (2004) [arXiv:hep-th/0312099].

[125] A. Kehagias and E. Kiritsis, "Mirage cosmology,” JHEP 9911, 022 (1999) [arXiv:hep-th/9910174].

[126] J. c. Hwang and E. T. Vishniac, "Gauge-invariant joining conditions for cosmological perturbations," Astrophys. J. 382, 363 (1991).

[127] N. Deruelle and V. F. Mukhanov, “On matching conditions for cosmological perturbations,” Phys. Rev. D 52, 5549 (1995) [arXiv:gr-qc/9503050].

[128] R. Durrer and F. Vernizzi, "Adiabatic perturbations in pre big bang models: Matching conditions and scale invariance,” Phys. Rev. D 66, 083503 (2002) [arXiv:hep-ph/0203275].

[129] Y. Cai, R. Brandenberger and X. Zhang, "The Matter Bounce Curvaton Scenario", arXiv:1101.0822 [hep-th].

[130] A. Cerioni and R. H. Brandenberger, "Cosmological Perturbations in the Projectable Version of Horava-Lifshitz Gravity,” arXiv:1007.1006 [hep-th].

[131] A. Cerioni and R. H. Brandenberger, "Cosmological Perturbations in the 'Healthy Extension” of Horava-Lifshitz gravity,” arXiv:1008.3589 [hep-th].

[132] Y. F. Cai, W. Xue, R. Brandenberger and X. Zhang, "Non-Gaussianity in a Matter Bounce," JCAP 0905, 011 (2009) [arXiv:0903.0631 [astro-ph.CO]].

[133] X. Chen, "Primordial Non-Gaussianities from Inflation Models," arXiv:1002.1416 [astro-ph.CO].

[134] J. M. Maldacena, "Non-Gaussian features of primordial fluctuations in single field inflationary models,” JHEP 0305, 013 (2003) [arXiv:astro-ph/0210603].

[135] H. Li, J. Q. Xia, R. Brandenberger and X. Zhang, "Constraints on Models with a Break in the Primordial Power Spectrum,” arXiv:0903.3725 [astro-ph.CO].

[136] N. Kaloper, A. D. Linde and R. Bousso, "Pre-big-bang requires the universe to be exponentially large from the very beginning," Phys. Rev. D 59, 043508 (1999) [arXiv:hep-th/9801073].

[137] G. F. R. Ellis and R. Maartens, "The emergent universe: Inflationary cosmology with no singularity," Class. Quant. Grav. 21, 223 (2004) [arXiv:gr-qc/0211082]. 
[138] J. M. Maldacena, “The large N limit of superconformal field theories and supergravity,” Adv. Theor. Math. Phys. 2, 231 (1998) [Int. J. Theor. Phys. 38, 1113 (1999)] [arXiv:hep-th/9711200].

[139] R. H. Brandenberger, “Challenges for string gas cosmology,” arXiv:hep-th/0509099.

[140] T. Battefeld and S. Watson, “String gas cosmology,” Rev. Mod. Phys. 78, 435 (2006) [arXiv:hep-th/0510022].

[141] S. Alexander, R. H. Brandenberger and D. Easson, "Brane gases in the early universe," Phys. Rev. D 62, 103509 (2000) [arXiv:hep-th/0005212].

[142] J. Polchinski, String Theory, Vols. 1 and 2, (Cambridge Univ. Press, Cambridge, 1998).

[143] T. Boehm and R. Brandenberger, "On T-duality in brane gas cosmology," JCAP 0306, 008 (2003) [arXiv:hep-th/0208188].

[144] K. Hotta, K. Kikkawa and H. Kunitomo, "Correlation between momentum modes and winding modes in Brandenberger-Vafa’s string cosmological model,” Prog. Theor. Phys. 98, 687 (1997) [arXiv:hep-th/9705099].

[145] M. A. R. Osorio and M. A. Vazquez-Mozo, “A Cosmological Interpretation Of Duality,” Phys. Lett. B 320, 259 (1994) [arXiv:hep-th/9311080].

[146] A. A. Tseytlin and C. Vafa, "Elements of string cosmology," Nucl. Phys. B 372, 443 (1992) [arXiv:hep-th/9109048].

[147] G. Veneziano, "Scale factor duality for classical and quantum strings," Phys. Lett. B 265, 287 (1991).

[148] A. A. Tseytlin, "Dilaton, winding modes and cosmological solutions," Class. Quant. Grav. 9, 979 (1992) [arXiv:hep-th/9112004].

[149] R. H. Brandenberger, A. R. Frey and S. Kanno, "Towards A Nonsingular Tachyonic Big Crunch," Phys. Rev. D 76, 063502 (2007) [arXiv:0705.3265 [hep-th]].

[150] S. Arapoglu, A. Karakci and A. Kaya, "S-duality in string gas cosmology," Phys. Lett. B 645, 255 (2007) [arXiv:hep-th/0611193].

[151] M. Sakellariadou, "Numerical Experiments in String Cosmology,” Nucl. Phys. B 468, 319 (1996) [arXiv:hep-th/9511075].

[152] G. B. Cleaver and P. J. Rosenthal, "String cosmology and the dimension of space-time," Nucl. Phys. B 457, 621 (1995) [arXiv:hep-th/9402088].

[153] R. Brandenberger, D. A. Easson and D. Kimberly, "Loitering phase in brane gas cosmology," Nucl. Phys. B 623, 421 (2002) [arXiv:hep-th/0109165].

[154] R. Easther, B. R. Greene, M. G. Jackson and D. Kabat, "String windings in the early universe," JCAP 0502, 009 (2005) [arXiv:hep-th/0409121].

[155] R. Danos, A. R. Frey and A. Mazumdar, “Interaction rates in string gas cosmology," Phys. Rev. D 70, 106010 (2004) [arXiv:hep-th/0409162].

[156] B. Greene, D. Kabat and S. Marnerides, "Dynamical Decompactification and Three Large Dimensions," arXiv:0908.0955 [hep-th].

[157] S. Watson and R. H. Brandenberger, "Isotropization in brane gas cosmology," Phys. Rev. D 67, 043510 (2003) [arXiv:hep-th/0207168].

[158] S. Watson and R. Brandenberger, "Stabilization of extra dimensions at tree level," JCAP 0311, 008 (2003) [arXiv:hep-th/0307044]. 
[159] S. P. Patil and R. Brandenberger, "Radion stabilization by stringy effects in general relativity and dilaton gravity,” Phys. Rev. D 71, 103522 (2005) [arXiv:hep-th/0401037].

[160] S. P. Patil and R. H. Brandenberger, “The cosmology of massless string modes,” JCAP 0601, 005 (2006) [arXiv:hep-th/0502069].

[161] S. Watson, “Moduli stabilization with the string Higgs effect,” Phys. Rev. D 70, 066005 (2004) [arXiv:hep-th/0404177].

[162] L. Kofman, A. Linde, X. Liu, A. Maloney, L. McAllister and E. Silverstein, "Beauty is attractive: Moduli trapping at enhanced symmetry points," JHEP 0405, 030 (2004) [arXiv:hep-th/0403001].

[163] R. H. Brandenberger, "Moduli stabilization in string gas cosmology,” Prog. Theor. Phys. Suppl. 163, 358 (2006) [arXiv:hep-th/0509159].

[164] R. Brandenberger, Y. K. Cheung and S. Watson, "Moduli stabilization with string gases and fluxes," JHEP 0605, 025 (2006) [arXiv:hep-th/0501032].

[165] R. J. Danos, A. R. Frey and R. H. Brandenberger, "Stabilizing moduli with thermal matter and nonperturbative effects,” Phys. Rev. D 77, 126009 (2008) [arXiv:0802.1557 [hep-th]].

[166] R. H. Brandenberger, A. Nayeri, S. P. Patil and C. Vafa, "String gas cosmology and structure formation,” Int. J. Mod. Phys. A 22, 3621 (2007) [arXiv:hep-th/0608121].

[167] R. H. Brandenberger, A. Nayeri, S. P. Patil and C. Vafa, "Tensor modes from a primordial Hagedorn phase of string cosmology," Phys. Rev. Lett. 98, 231302 (2007) [arXiv:hep-th/0604126].

[168] R. H. Brandenberger et al., "More on the spectrum of perturbations in string gas cosmology," JCAP 0611, 009 (2006) [arXiv:hep-th/0608186].

[169] N. Kaloper, L. Kofman, A. Linde and V. Mukhanov, "On the new string theory inspired mechanism of generation of cosmological perturbations,” JCAP 0610, 006 (2006) [arXiv:hep-th/0608200].

[170] N. Deo, S. Jain, O. Narayan and C. I. Tan, "The Effect of topology on the thermodynamic limit for a string gas," Phys. Rev. D 45, 3641 (1992).

[171] A. Nayeri, "Inflation free, stringy generation of scale-invariant cosmological fluctuations in D $=3+$ 1 dimensions," arXiv:hep-th/0607073.

[172] B. Chen, Y. Wang, W. Xue and R. Brandenberger, "String Gas Cosmology and Non-Gaussianities," arXiv:0712.2477 [hep-th].

[173] E. Witten, “Cosmic Superstrings,” Phys. Lett. B 153, 243 (1985).

[174] N. Kaiser and A. Stebbins, “Microwave Anisotropy Due To Cosmic Strings," Nature 310, 391 (1984).

[175] S. Amsel, J. Berger and R. H. Brandenberger, "Detecting Cosmic Strings in the CMB with the Canny Algorithm,” JCAP 0804, 015 (2008) [arXiv:0709.0982 [astro-ph]];

A. Stewart and R. Brandenberger, "Edge Detection, Cosmic Strings and the South Pole Telescope," arXiv:0809.0865 [astro-ph];

R. J. Danos and R. H. Brandenberger, "Canny Algorithm, Cosmic Strings and the Cosmic Microwave Background," arXiv:0811.2004 [astro-ph];

R. J. Danos and R. H. Brandenberger, "Searching for Signatures of Cosmic Superstrings in the CMB," arXiv:0910.5722 [astro-ph.CO].

[176] N. Kaloper and S. Watson, "Geometric Precipices in String Cosmology,” Phys. Rev. D 77, 066002 (2008) [arXiv:0712.1820 [hep-th]]. 
[177] T. Biswas, R. Brandenberger, A. Mazumdar and W. Siegel, "Non-perturbative gravity, Hagedorn bounce and CMB," JCAP 0712, 011 (2007) [arXiv:hep-th/0610274].

[178] R. A. Sunyaev, Y. .B. Zeldovich, "Small scale fluctuations of relic radiation," Astrophys. Space Sci. 7, 3-19 (1970).

[179] P. J. E. Peebles, J. T. Yu, “Primeval adiabatic perturbation in an expanding universe,” Astrophys. J. 162, 815-836 (1970). 